Draft VERsion November 4, 2016

Preprint typeset using $\mathrm{LAT}_{\mathrm{E}} \mathrm{X}$ style AASTeX6 v. 1.0

\title{
A NEW ELECTRON DENSITY MODEL FOR ESTIMATION OF PULSAR AND FRB DISTANCES
}

\author{
J. M. YAO ${ }^{1,3}$, R. N. MANCHESTER ${ }^{2}$, AND N. WANG ${ }^{1,4}$ \\ ${ }^{1}$ Xinjiang Astronomical Observatory, Chinese Academy of Sciences, 150, Science 1-Street, Urumqi, Xinjiang 830011, China; \\ ${ }^{2}$ CSIRO Astronomy and Space Science, Australia Telescope National Facility, P.O. Box 76, Epping NSW 1710, Australia \\ ${ }^{3}$ University of Chinese Academy of Sciences, 19A Yuquan Road, Beijing 100049, China \\ ${ }^{4}$ Key Laboratory of Radio Astronomy, Chinese Academy of Science, Nanjing 210008, China
}

\begin{abstract}
We present a new model for the distribution of free electrons in the Galaxy, the Magellanic Clouds and the intergalactic medium (IGM) that can be used to estimate distances to real or simulated pulsars and fast radio bursts (FRBs) based on their dispersion measure (DM). The Galactic model has an extended thick disk representing the so-called warm interstellar medium, a thin disk representing the Galactic molecular ring, spiral arms based on a recent fit to Galactic HII regions, a Galactic Center disk and seven local features including the Gum Nebula, Galactic Loop I and the Local Bubble. An offset of the Sun from the Galactic plane and a warp of the outer Galactic disk are included in the model. Parameters of the Galactic model are determined by fitting to 189 pulsars with independently determined distances and DMs. Simple models are used for the Magellanic Clouds and the IGM. Galactic model distances are within the uncertainty range for 86 of the 189 independently determined distances and within $20 \%$ of the nearest limit for a further 38 pulsars. We estimate that $95 \%$ of predicted Galactic pulsar distances will have a relative error of less than a factor of 0.9 . The predictions of YMW16 are compared to those of the TC93 and NE2001 models showing that YMW16 performs significantly better on all measures. Timescales for pulse broadening due to interstellar scattering are estimated for (real or simulated) Galactic and Magellanic Cloud pulsars and FRBs.
\end{abstract}

Keywords: pulsars:general — stars:distances — ISM:structure

\section{INTRODUCTION}

Distances to astronomical objects are often difficult to estimate, but are of fundamental significance. They determine, for example, the source luminosity, the location in the Galaxy or the Universe and the space velocity, all important for studies of the origin, evolution and emission properties of the object in question. Pulsars come with a built-in distance indicator, interstellar dispersion, which results in a radio-frequency-dependent delay $\Delta t$ in the pulse arrival times:

$$
\Delta t=\frac{e^{2}}{2 \pi m c} \nu^{-2} \int_{0}^{D} n_{e} d l
$$

where $e$ and $m$ are the charge and mass of the electron, $c$ is the velocity of light, $\nu$ is the radio frequency, $n_{e}$ is the local free electron density and the integral is along the path to the pulsar at distance $D$. The electron column density along the path is known as the dispersion measure (DM) and can be measured using:

$$
\mathrm{DM}=\int_{0}^{D} n_{e} d l=2.410 \times 10^{-16}\left(t_{2}-t_{1}\right) /\left(\nu_{2}^{-2}-\nu_{1}^{-2}\right) \mathrm{cm}^{-3} \mathrm{pc}
$$

where $t_{1}$ and $t_{2}$ are observed pulse arrival times at frequencies $\nu_{1}$ and $\nu_{2}$, respectively.

Most of the $\sim 2540$ currently known pulsars ${ }^{1}$ are located within our Galaxy - the 29 known extra-galactic pulsars are all in the Magellanic Clouds. Of the known pulsars, $\sim 2430$ in the Galaxy and 27 in the Magellanic Clouds have known DMs (the remainder were discovered at high energies and currently have no radio counterpart). Figure 1 shows the distribution of DMs as a function of Galactic longitude $l$.

\footnotetext{
1 See the ATNF Pulsar Catalogue: http://www.atnf.csiro.au/research/pulsar/psrcat, V1.54. Note that this total includes rotating radio transients (RRATs) even when no pulsational period has been identified, anomalous X-ray pulsars (AXPs), soft-gamma-ray repeaters (SGRs) with detected periodic pulsations, and X-ray isolated neutron stars (XINS).
} 




Figure 1. Dispersion measure plotted against Galactic longtitude for the 2430 Galactic pulsars with known DM. As will be discussed further below, the line is the total DM obtained by integrating the YMW16 electron density model through the Galaxy at $b=0$.

Fast radio bursts (FRBs) are isolated millisecond-duration radio bursts that have DMs that are much higher than expected from the Galaxy, suggesting an extra-galactic origin. The first FRB, discovered by Lorimer et al. (2007), remained enigmatically alone for several years, but in the past few years many more have been detected ${ }^{2}$, firmly establishing them as an extra-galactic phenomenon. One FRB has been found to emit repeated pulses (Spitler et al. 2016), suggesting a relationship with pulsars, especially RRATS (cf., Keane \& Petroff 2015), but their origin remains a topic of great debate. However, this uncertainty about the nature of the source does not affect use of their DM as a distance indicator.

To make use of DMs for pulsar or FRB distance estimation, a model for the distribution of free electrons in the intervening interstellar medium (ISM) or intergalactic medium (IGM) is required. In principle, at least for our Galaxy and nearby galaxies, such a model could be derived directly from observations of recombination lines of ionised interstellar gas or radio thermal continuum radiation. In practice though, this is difficult. The intensity of both these types of radiation is related to the emission measure (EM), which is proportional to the integral of $n_{e}^{2}$ along the path. Since the filling factor $\left\langle n_{e}^{2}\right\rangle /\left\langle n_{e}\right\rangle^{2}$ is largely unknown and variable along typical interstellar paths, conversion from EM to DM is problematic. Observed recombination lines and radio thermal continuum are dominated by regions of high density such as HII regions, whereas, except at low Galactic latitudes, pulsar dispersion largely originates in the more widely distributed "warm ionized medium" (WIM) which can be investigated using wide-field H $\alpha$ observations (e.g., Haffner et al. 2003). Even these observations are not useful for inner regions of the Galaxy since they are affected by interstellar dust extinction which is difficult to quantify, especially when optical depths become high.

The main components of a reasonable Galactic $n_{e}$ model can be identified from our general knowledge of the distribution of molecular gas and star-formation regions in the Galaxy, as well as $\mathrm{H} \alpha$ surveys of more local gas. For example, these can be used to define the spiral-arm structure in the Galaxy (e.g., Hou \& Han 2014) and suggest the presence of a "thick" electron disk with scale height $\sim 1.5 \mathrm{kpc}$ in addition to the "thin" disk defined by HII regions which has a scale height $\sim 70$ pc (e.g., Hill et al. 2008). However, the parameters describing the $n_{e}$ distribution generally cannot be independently determined with sufficient precision. Hence we are forced to use the observed DMs of pulsars with independently known distances to calibrate the $n_{e}$ model. As is discussed further in $\S 2$ below, such independent distances can be obtained from measurements of pulsar parallax, pulsar associations with globular clusters or supernova remnants, optical observations of binary companions and kinematic distances based on observations of 21-cm HI absorption in pulsar spectra combined with a model for Galactic rotation.

An accurate model for the $n_{e}$ distribution in the Galaxy has other important uses besides the direct estimation of pulsar distances. For example, it can help to identify pulsars that are likely to be in other galaxies such as the Magellanic Clouds by their excess DM over that expected from the Galaxy (e.g., Manchester et al. 2006), and to firmly establish that "fast radio bursts" (FRBs) are distant extra-galactic sources (e.g., Thornton et al. 2013). A knowledge of the $n_{e}$ distribution along the path is important for the interpretation of Faraday rotation in pulsars and extra-galactic

${ }^{2}$ For a catalog of currently known FRBs, see http://www.astronomy.swin.edu.au/pulsar/frbcat/ and Petroff et al. (2016) 
sources when investigating the large-scale structure of the Galactic magnetic field (e.g., Han et al. 2006; Van Eck et al. 2011) and for the interpretation of measurements of interstellar scattering (e.g., Bower et al. 2014; Lewandowski et al. 2015). It is also important in investigations of the intergalactic medium, especially intergalactic magnetic fields (e.g., Pshirkov et al. 2015).

One of the first attempts to construct a Galactic $n_{e}$ model was by Manchester \& Taylor (1981) and Lyne et al. (1985). Their model (LMT85) consisted of two main components: a thin disk of scale height $70 \mathrm{pc}$ and a $z$-independent component (where $z$ is the perpendicular distance from the Galactic plane), both of which had a mid-plane electron density that tapered off with increasing Galactocentric radius $R$. In addition, the Gum Nebula (Gum 1952) was recognised as significantly modifying the DMs of pulsars lying within or behind it and hence was included in the model as a separate term. The model was calibrated using the 36 pulsars with independent distances known at the time, mostly kinematic distances from HI absorption. At about the same time Vivekanand \& Narayan (1982) used data from the Second Molonglo pulsar survey to investigate the form of the $n_{e}$ distribution at $R>5 \mathrm{kpc}$, concluding that, apart from an HII-region layer, the disk thickness was large. They presented a simple model that is very similar to the LMT85 model.

The next major step forward was by Taylor \& Cordes (1993), building on the work of Cordes et al. (1991), who included the effect of spiral structure in the model and also made use of the increased number of independent distances (74) and the overall dependence of pulsar DMs on Galactic longitude. For the first time, they also took into account observations of interstellar scattering. Their "TC93" model included both thin and thick Galactic disks and made use of the $\operatorname{sech}^{2}(x)$ function ${ }^{3}$ for the $z$-dependencies and some $r$-dependencies of $n_{e}$ (see also Gómez et al. 2001). The $\operatorname{sech}^{2}(x)$ function has a physical basis (see Spitzer 1942) and (unlike the exponential function) has no cusp at $x=0$, but is asymptotically exponential at large $x$.

Since 2002, the "NE2001" model (Cordes \& Lazio 2002; Cordes \& Lazio 2003) has been the de facto standard for estimation of pulsar distances. This model makes use of 112 independent pulsar distances and scattering measures (SMs) for 269 pulsars to define a model which includes both a quasi-smooth $n_{e}$ distribution and large-scale variations in the strength of the fluctuations in $n_{e}$ that result in interstellar scattering. The model includes multiple components: thin and thick axisymmetric disks, spiral arms, and local components including a local arm, a local hot bubble surrounding the Sun and relatively large super-bubbles in the first and third Galactic quadrants, together with overdense components representing the Gum Nebula, the Vela supernova remnant, Galactic Loop I and a small region around the Galactic Center. Finally, NE2001 adds clumps toward pulsars with excess DM or scattering and toward active galactic nuclei (AGNs) that have excess scattering, and voids toward pulsars that have DMs below those predicted by the quasi-smooth component. The model also uses $\operatorname{sech}^{2}(z)$ dependencies and includes 67 parameters describing the main components, including parameters that describe the $n_{e}$ fluctuation amplitudes in each component. Another 493 parameters were used to describe 82 clumps and 137 parameters to describe 17 voids. An iterative approach was used to fit the parameters to the available data, first solving for the parameters of the large-scale quasi-smooth components, then adding the clumps and voids and other local features and refitting as necessary (Cordes \& Lazio 2002; Cordes \& Lazio 2003).

Compared to the TC93 model, distances obtained from NE2001 are generally smaller, especially for high-latitude pulsars which in many cases had unbounded distances in the TC93 model. This mainly results from NE2001 having a denser thick disk $\left(n_{1_{0}}=0.035 \mathrm{~cm}^{-3}, H_{1}=950 \mathrm{pc}\right)$ compared to TC93 $\left(n_{1_{0}}=0.019 \mathrm{~cm}^{-3}, H_{1}=880 \mathrm{pc}\right)$, where $n_{1_{0}}$ is the mid-plane electron density for the thick disk and $H_{1}$ is its scale height.

In the next few years, the population of known pulsars increased significantly, mainly as a result of the Parkes Multibeam Pulsar Survey, and several groups (e.g., Lorimer et al. 2006) began to notice that NE2001 systematically under-estimates the $z$-distance of high-latitude pulsars. To address this, Gaensler et al. (2008) analysed just highlatitude pulsars $\left(|b|>40^{\circ}\right)$ that are not contaminated by HII regions or spiral arms along the path. They obtained a smaller mid-plane density, $n_{1_{0}}=0.014 \pm 0.001 \mathrm{~cm}^{-3}$, and a much larger (exponential) scale height $H_{1}=1830_{-250}^{+120} \mathrm{pc}$. However, Savage \& Wakker (2009) pointed out a bias in the fitting method used by Gaensler et al. (2008) and obtained a revised scale height of $1410_{-210}^{+260} \mathrm{pc}$. For both the exponential and $\operatorname{sech}^{2}(z)$ distributions, the $n_{1_{0}} H_{1}$ product is equal to the "perpendicular DM" $\left(\mathrm{DM}_{\perp}\right)$, i.e., the DM integrated to infinity perpendicular to the Galactic disk. For the TC93, N2001, Gaensler et al. (2008) and Savage \& Wakker (2009) fits, $\mathrm{DM}_{\perp}$ is, respectively, $16.5 \mathrm{~cm}^{-3} \mathrm{pc}, 33.0 \mathrm{~cm}^{-3} \mathrm{pc}$, $25.6 \mathrm{~cm}^{-3} \mathrm{pc}$ and $21.9 \mathrm{~cm}^{-3}$ pc. Gaensler et al. (2008) also estimated a mid-plane volume filling factor $\left(f_{v}\right)$ for the

$$
{ }^{3} \operatorname{sech}^{2}(x)=1 / \cosh ^{2}(x)=4 /\left(e^{x}+e^{-x}\right)^{2}
$$


thick-disk electrons of 0.014 , rising to around 0.3 at $z \sim 1000$ pc. $^{4}$

Based on accurate parallax measurements using the VLBA, Chatterjee et al. (2009) also showed that the NE2001 model under-predicts distances for several high-latitude pulsars by a factor of two, but also over-predicts distances for several relatively local pulsars. These results illustrate the clumpy nature of the ISM on scales of $100-1000$ pc. An analysis by Schnitzeler (2012) considered a range of modified TC93 and NE2001 models with different parameters for the thick disk. These were tested using a sample of 41 pulsars with $|b|>5^{\circ}$, having well-determined independent distances and unaffected by HII regions in the path. A modified TC93 model with a scale height of $1590 \pm 300 \mathrm{pc}$ and the same $n_{1_{0}} H_{1}$ product as TC93 gave the most consistently accurate predictions.

In this work we present a new "YMW16" model for the large-scale distribution of free electrons in the Galaxy, the Magellanic Clouds and the intergalactic medium. The Galactic part of the model has the same basic structure as TC93 and NE2001 but also some important differences compared to these earlier models. For example, we adopt a four-armed spiral pattern (plus a "local arm") with the location and form of the arms as given by Hou \& Han (2014) based on observations of more than 1800 HII regions across the Galaxy. The YMW16 model is fitted to a compilation of 189 independent pulsar distance estimates, using global and local optimization algorithms. There are seven local features in the YMW16 model: the Local Bubble (LB), two regions of enhanced $n_{e}$ on the periphery of the Local Bubble (LB1, LB2), the Gum Nebula (GN), Loop I (LI), a region of enhanced $n_{e}$ in the Carina arm and a region of reduced $n_{e}$ in the Sagittarius tangential region. Most of these features are also included in the NE2001 model.

In contrast to both TC93 and NE2001, we do not make use of observations of interstellar scattering in building the model. Numerous observations (e.g., Stinebring 2006; Trang \& Rickett 2007; Brisken et al. 2010) have shown that interstellar scattering is typically dominated by just a few regions of very strong $n_{e}$ fluctuations along the path to a pulsar. This makes it essentially impossible to satisfactorily model the large-scale distribution of interstellar scattering and leads to the very large scatter seen in plots of SM and scattering delay $\tau_{\text {sc }}$ versus DM (e.g., Lewandowski et al. 2015; Krishnakumar et al. 2015).

Another major difference between NE2001 and YMW16 is that we do not attempt to correct discrepant model distances for individual pulsars by invoking clumps or voids in their direction. We believe that this procedure should be deprecated as it is likely to lead to poor distance estimates for future discoveries (or even for currently known pulsars) that have adjacent lines of sight. We have only invoked additional features beyond the large-scale model where a number of pulsars in a given region have discrepant distances and/or where there is good independent evidence for such features. This policy of course leads to more pulsars having over-estimated or under-estimated distances, but this is an unavoidable consequence of our current inability to adequately model the small-scale structure in the ISM.

With the advent of FRB astronomy and the likely increase in the number and distribution of extra-galactic pulsars with increasingly sensitive searches, there is a strong motivation to include extra-galactic components in the electrondensity model. We model the DM contributions of the Large and Small Magellanic Clouds (LMC, SMC) based on prior information on their shape, size and distance and making use of the DMs of the 27 pulsars believed to be associated with either the LMC or SMC. We also include components for the IGM and the host galaxy to allow distance estimates for FRBs.

The arrangement of our paper is as follows. Pulsars with DM-independent distance estimates or limits and the methods by which these are obtained are discussed in $\S 2$. In $\S 3$ we describe the functional form of the model components and in $\S 4$ we describe the model fitting procedure and the algorithms used for global optimization and local optimization. Results from the model fitting are presented and compared with the predictions of the TC93 and NE2001 models in $\S 5$. A summary our results and concluding remarks are given in $\S 6$. Tables of DM-independent distances and limits, and corresponding model distances are given in Appendix A. Details of the algorithms used to compute perpendicular distances from spiral arms and the Gum Nebula are given in Appendix B. Coordinate conversions for the LMC are given in Appendix C and the YMw16 program code and outputs are described in Appendix D.

\section{MODEL-INDEPENDENT DISTANCES}

There are a variety of ways that pulsar distances can be estimated independent of their DM and the Galactic $n_{e}$ model. As described above, these independent distances are essential for the calibration of any model for the $n_{e}$ distribution. We have obtained independent distance data for 189 pulsars from the ATNF Pulsar Catalogue (V1.54), only including pulsars with both upper and lower limits on the estimated distance and known DMs. Only one pulsar from each globular cluster association (and the Double Pulsar) is included. Parallax measurements of low significance

\footnotetext{
4 The volume filling factor $\left(f_{v}\right)$ is related to the line-of-sight filling factor $\left\langle n_{e}^{2}\right\rangle /\left\langle n_{e}\right\rangle^{2}$ by a form-dependent factor which is of order unity (Berkhuijsen et al. 2006).
} 
are also omitted. In this section we discuss the different methods of obtaining independent distances; the adopted distances are listed in Appendix A for each category.

\subsection{Distances from annual parallax}

Measurements of annual parallax give a direct measure of the pulsar distance. Such measurements can be made in two different ways: a) fitting for annual parallax in precision timing solutions and b) direct measurements of position shifts due to annual parallax using very-long-baseline interferometry (VLBI). Of the 70 parallax measurements listed in Table A1, about 40 are based on VLBI observations, one (PSR J0633+1746) on optical astrometry with the Hubble Space Telescope, and the remainder on pulse timing analyses. Measured parallaxes of low significance are biased by the "Lutz-Kelker" effect (Lutz \& Kelker 1973). This has been considered in the context of pulsar parallax measurements by Verbiest et al. (2012) and we have adopted their corrected distance estimates where available. Otherwise, we have ignored parallax measurements with a value less than three times the quoted uncertainty.

For three of the entries in Table A1 (PSRs J0437-4715, J1537+1155 and J2129-5721), the distance estimate is based on a measure of the time-derivative of the orbital period $\dot{P}_{b}$. If a signficant excess $\dot{P}_{b}$ (over that expected from general-relativistic decay of the orbit) is measured, then this excess can be attributed to the Shklovskii effect (Shklovskii 1970) and a distance estimate obtained (Bell \& Bailes 1996). For these three pulsars, the $\dot{P}_{b}$ distance is the most precise distance estimate available.

\subsection{Kinematic distances}

For low-latitude pulsars, 21-cm absorption spectra resulting from dense HI clouds in the path can be used to obtain pulsar distance limits by using a Galactic rotation model to convert radial velocities of clouds to distances. Lower and upper distance limits correspond to the presence and absence of absorption features, but upper distance limits are always more difficult to estimate. Also for "inner" lines of sight $\left(0^{\circ}<l<90^{\circ}\right.$ and $\left.270^{\circ}<l<360^{\circ}\right)$ difficulties arise due to ambiguities about whether a given spectral feature corresponds to absorbing gas at the "near" distance (closer than the tangent point) or the "far" distance (beyond the tangential point). Generally, emission features with $T_{B} \gtrsim 35 \mathrm{~K}$ correspond to significant absorption and this has been used a criterion to decide on distance limits (Weisberg et al. 1979; Frail \& Weisberg 1990). Table A2 gives kinematic distances derived from HI absorption and emission spectra for 62 pulsars. Parallax distances are always underestimated because of the Lutz-Kelker bias but kinematic distances tend to be over-estimated because of a luminosity bias. Again, this bias was considered by Verbiest et al. (2012) and we have adopted their limits when available. Because of the difficulties in interpretation of observed spectra, HI kinematic distances are generally less reliable than those derived using other methods.

\subsection{Association with globular clusters}

Many (mostly) millisecond pulsars (MSPs) are associated with globular clusters - it is clear that the dense cores of these clusters have conditions that are favourable for the recycling of pulsars to form MSPs. Distances to clusters are obtained by a variety of methods including astrometry (e.g., McLaughlin et al. 2006a) and photometry (e.g., Ortolani et al. 2007). In our database we include just one pulsar for each cluster, but assign to it a DM averaged over the pulsars in that cluster. Table A3 lists the 27 clusters and pulsars for which distances have been obtained from observations of the associated globular cluster. Globular clusters are all at large distances from the Sun and these associations are of great importance in defining the distance model, especially for larger $z$-distances.

\subsection{Association with nebulae}

About 60 young pulsars are associated with supernova remnants (SNR) but many of these were discovered at X-ray or $\gamma$-ray wavelengths and have no radio counterpart. Consequently their DM is unknown and they are not useful as independent distances for $n_{e}$ modelling. Table A4 lists 14 pulsars that are associated with SNR or pulsar wind nebulae (PWN), have a known DM and an estimated distance based on the association. In this Table we also include a pulsar (PSR J0248+6021) that is believed to lie in or near the HII region W5 (Theureau et al. 2011) and the magnetar PSR J1745-2900 that is believed to lie close to the Galactic Center (Eatough et al. 2013; Rea et al. 2013).

\subsection{Binary companion stars}

Although about 250 pulsars are members of a binary system, in orbit with another star, in only a few cases is the companion star optically identified. Table A5 gives the nine published independent distance estimates based on optical identifications. 


\subsection{Extra-galactic pulsars}

Up to now, pulsars believed to lie outside our Galaxy have only been found in the Magellanic Clouds, our nearest neighbor galaxies. A total of 29 pulsars are known in the Clouds, six in the SMC and 23 in the LMC. However, only 27 of these have known DM, with one pulsar in each of the SMC and LMC being detected only at high energies. These 27 pulsars are listed in Table A6 along with their nominal distance estimates and limits which refer to the center of each Cloud. The Magellanic Clouds form an important step in the extra-galactic distance ladder and so extensive studies of Cepheid and RR Lyrae variables have been carried out in order to estimate their distances. For the LMC we have adopted the distance modulus of $18.48 \pm 0.05$ given by Walker (2012), which corresponds to a distance of $49.7 \pm 1.1 \mathrm{kpc}$. For the SMC, we adopt the value from Storm et al. (2004): distance modulus $18.88 \pm 0.13$ corresponding to a distance of $59.7_{-3.5}^{+3.8} \mathrm{kpc}$.

\subsection{Fast radio bursts}

Currently, there are 17 known FRBs as listed in Table A7 (Petroff et al. 2016). Only one of these, FRB150418, has an identification of the host galaxy, an elliptical galaxy at $z=0.492 \pm 0.008$ (Keane et al. 2016), although this identification has been disputed (Akiyama \& Johnson 2016; Williams \& Berger 2016).

\section{COMPONENTS OF THE MODEL}

In this section we describe the various components of the YMW16 model for the distribution of free electrons in the Galaxy, the Magellanic Clouds and the intergalactic medium. We also describe the model for interstellar scattering delays. For the Galaxy we use a right-handed coordinate system with origin at the Galactic Center, $x$ axis parallel to $l=90^{\circ}$ and $y$ axis parallel to $l=180^{\circ}$ where $(l, b)$ are the usual Sun-centred Galactic coordinates. The Sun is located at $\left(x=0, y=R_{\odot}, z=z_{\odot}\right)$. We adopt a distance of the Sun from the Galactic Center, $R_{\odot}=8300 \mathrm{pc}$ (Brunthaler et al. $2011)^{5}$. Recent estimates of $z_{\odot}$ range between $+6 \mathrm{pc}$ and $+30 \mathrm{pc}$ (Joshi et al. 2016). Based on an analysis of nearby open clusters Joshi et al. (2016) obtain $z_{\odot}=+6.2 \pm 1.1 \mathrm{pc}$; we fix $z_{\odot}$ at $+6.0 \mathrm{pc}$. The Galactic Cartesian coordinates of any object are $(x, y, z)=\left(D \sin l \cos b, R_{\odot}-D \cos l \cos b, z_{\odot}+D \sin b\right)$, where $D$ is its distance from the Sun. We also use the Galactocentric cylindrical coordinate system $(R, \phi, z)$, which is defined with $R=\left(x^{2}+y^{2}\right)^{1 / 2}$ and $\phi$ measured counter-clockwise from the $+x$ direction, i.e., toward the $+y$ direction.

There is extensive evidence from HI surveys (see Kalberla \& Kerp 2009, for a review) and stellar distributions (e.g., Urquhart et al. 2014) that the Galactic disk has a pronounced warp in its outer regions, most probably induced by gravitational interactions with the Magellanic Clouds. Robin et al. (2003) have modelled the warp as follows:

$$
\begin{gathered}
z_{w}=z_{c} \cos \left(\phi-\phi_{w}\right), \\
z_{c}=\gamma_{w}\left(R-R_{w}\right),
\end{gathered}
$$

with the parameters $\phi_{w}=0^{\circ},{ }^{6}$ where we take $R_{w}=8400 \mathrm{pc}$ and fit for $\gamma_{w}$. That is, the warp begins just outside the solar radius and is toward positive $z$ in the $+x$ direction.

\subsection{Thick disk}

The presence of an extensive diffuse ionised medium in the disk of our Galaxy was proposed more than five decades ago by Hoyle \& Ellis (1963) based on evidence for free-free absorption in the spectrum of the Galactic synchrotron background. Further evidence came from observations of diffuse $\mathrm{H} \alpha$ and other recombination lines (Reynolds et al. 1973; Haffner et al. 1999; Madsen et al. 2006), pulsar DMs (Manchester \& Taylor 1981; Nordgren et al. 1992; Cordes \& Lazio 2002; Gaensler et al. 2008; Savage \& Wakker 2009; Schnitzeler 2012) and interstellar scattering (Readhead \& Duffett-Smith 1975; Cordes et al. 1991; Cordes \& Lazio 2002), all suggesting mid-plane electron densities of $0.02-0.03 \mathrm{~cm}^{-3}$ and scale heights of $\sim 1000 \mathrm{pc}$ with no strong dependence of either mid-plane $n_{e}$ or scale height on Galactocentric radius out to the edge of the Galactic disk. Haffner et al. (1999) used observations of diffuse H $\alpha$ from the Perseus arm region, about $2.5 \mathrm{kpc}$ from the Sun at Galactic longitudes $125^{\circ}-150^{\circ}$, corresponding to $R \sim 10.5 \mathrm{kpc}$, to estimate a scale height $H_{n_{e}}$ for the WIM in this region of $1000 \pm 100 \mathrm{pc}$. This estimate assumes a constant filling factor $f_{v}$ for the ionised gas, so that $H_{n_{e}}=2 H_{n_{e}^{2}}$. Hill et al. (2014) used similar methods to investigate the WIM in the vicinity of the

\footnotetext{
${ }^{5}$ Both Taylor \& Cordes (1993) and Cordes \& Lazio (2002) used $R_{\odot}=8500$ pc. We ignore the small systematic offset introduced by this difference.

${ }^{6}$ Note that Robin et al. (2003) measure $\phi$ counterclockwise from the $-y$ direction, whereas we measure it counterclockwise from the $+x$ direction.
} 
Scutum-Centaurus arm at a distance of $3.5 \pm 0.3 \mathrm{kpc}$ from the Sun at Galactic longitudes $320^{\circ}-340^{\circ}$, corresponding to $R \sim 5 \mathrm{kpc}$. They estimate an $n_{e}^{2}$ scale height for the WIM in this region of about $430 \mathrm{pc}$, corresponding to $H_{n_{e}} \sim 860 \mathrm{pc}$ given the same assumptions. These results suggest that there is no strong dependence of $H_{n_{e}}$ on Galactocentric radius out to the edge of the Galactic disk. They also support the relative independence of WIM H $\alpha$ intensity and hence of $n_{e}$ as a function of Galactocentric radius. Preliminary fits to the independent distance data including $R$-dependencies of $n_{e}$ and/or $H_{n_{e}}$ were less successful than those omitting them.

We therefore model the thick disk as a plane-parallel disk of scale height $H_{1}$ :

$$
n_{1}=n_{1_{0}} g_{d} \operatorname{sech}^{2}\left(\frac{z-z_{w}}{H_{1}}\right)
$$

where $n_{1_{0}}$ is the mid-plane electron density and $H_{1}$ is the scale height. The extent of the Galactic disk is defined by the parameters $A_{d}$ and $B_{d}$ which are respectively the scale length of the cutoff and the Galactocentric radius at which the cutoff begins. The factor $g_{d}=1$ for $R<B_{d}$, and

$$
g_{d}=\operatorname{sech}^{2}\left(\frac{R-B_{d}}{A_{d}}\right)
$$

for $R \geq B_{d}$.

The radial extent of the thick disk (and other disk components) affects the model distance of distant pulsars and FRBs, especially those located toward the Galactic anticenter region. We have no independent distances for pulsars with $R>15 \mathrm{kpc}$ and so we cannot calibrate the cutoff radius in this way. While there is ample evidence of Galactic HI extending to $R \sim 20 \mathrm{kpc}$ (e.g., McClure-Griffiths et al. 2004), the distribution of young stars that could ionize this gas is evidently more limited. A survey of distant HII regions by Anderson et al. (2015) finds the last significant concentration peaking at $R \sim 12 \mathrm{kpc}$, with only a few at $R>15 \mathrm{kpc}$. We therefore fix $B_{d}$ at $15 \mathrm{kpc}$ and $A_{d}$ at $2.5 \mathrm{kpc}$, which results in the disk density being reduced to $\sim 15 \%$ of its $R=15 \mathrm{kpc}$ value at $R \sim 19 \mathrm{kpc}$. This allows for some ionisation beyond most of the known HII regions.

Despite the assumption of a plane-parallel thick disk, there are good reasons to suggest that the density of the thick disk is reduced in the vicinity of the Galactic Center. Firstly, there is a group of globular cluster pulsars with $|l| \lesssim 10^{\circ}$, $|b| \gtrsim 10^{\circ}$ at distances comparable to the Galactic Center, e.g., PSR J1835-3259A in NGC6652 and PSR J1823-3021A in NGC6624, for which the mean $n_{e}$ along the path is less than the nominal mid-plane density of the thick disk. Secondly, observations of the so-called "Fermi Bubbles" (see, e.g., Crocker et al. 2015, and references therein) indicate that powerful winds from the Galactic Center region have evacuated large bubbles extending to $z$-distances of $\sim 8 \mathrm{kpc}$ on both sides of the Galactic plane. In a slight modification of the prescription given by Su et al. (2010), we model the Fermi Bubbles as ellipsoidal cavities in the thick disk that extend to $l= \pm 20^{\circ}$ and $b= \pm 50^{\circ}$ and touch at the Galactic centre. The semi-major and semi-minor axes of each ellipsoid are therefore $a_{\mathrm{FB}}=0.5 R_{\odot} \tan 50^{\circ}$ and $b_{\mathrm{FB}}=R_{\odot} \tan 20^{\circ}$ respectively, and their centers are at $\left(x_{\mathrm{FB}}, y_{\mathrm{FB}}, z_{\mathrm{FB}}\right)=\left(0,0, \pm 0.5 R_{\odot} \tan 50^{\circ}\right)$, respectively. The boundary of each bubble is then defined by:

$$
P_{\mathrm{FB}}=\left(\frac{x}{b_{\mathrm{FB}}}\right)^{2}+\left(\frac{y}{b_{\mathrm{FB}}}\right)^{2}+\left(\frac{z-z_{\mathrm{FB}}}{a_{\mathrm{FB}}}\right)^{2}=1 .
$$

If $P_{\mathrm{FB}}<1$, then $n_{1}$ is replaced by $n_{1}^{\prime}=J_{\mathrm{FB}} n_{1}$, where $J_{\mathrm{FB}}$ is a constant scaling factor relating the electron density inside the bubbles to the unperturbed density of the thick disk at the same position.

\subsection{Thin disk}

As for the earlier TC93 and NE2001 models, the thin disk in our model represents the region of high gas density and massive star formation often referred to as the "molecular ring". Studies of the distribution of massive stars, molecular gas and HII regions in the inner Galaxy (e.g., Nakanishi \& Sofue 2006; Urquhart et al. 2014; Anderson et al. 2015) show that the gas density peaks at a Galactocentric radius of $4-5 \mathrm{kpc}$. Analyses of the $z$-distribution of tracers of high-density gas in the Galactic disk and spiral arms such as neutral hydrogen (HI) (e.g., Kalberla et al. 2007) and molecular gas (e.g., Nakanishi \& Sofue 2006) give scale heights in the range 50 - 70 pc at Galactocentric radii of 4 $6 \mathrm{kpc}$, whereas mid-infrared observations of massive stars in the Galactic disk (e.g., Urquhart et al. 2014) and open star clusters (Joshi et al. 2016) give scale heights less than half as large, $20-25$ pc. $^{7}$ All of these tracers show an

\footnotetext{
7 The thickness of the Galactic disk is parameterised by different authors in different ways. An exponential distribution with scale height $h_{e}$, i.e., $n_{e} \sim \exp \left(-|z| / h_{e}\right)$, is often assumed, whereas studies of the distribution of atomic and molecular gas generally give either the full width at half-maximum or the half-width at half-maximum (HWHM). In this paper we use the square of the hyperbolic secant, $\operatorname{sech}^{2}\left(|z| / h_{s}\right)$ to represent $z$-distributions (as well as some other distributions). The different definitions of scale height are significantly different with HWHM $=h_{e} \ln (2) \sim 0.693 h_{e}$ and HWHM $=h_{s} \ln (\sqrt{2}+1) \sim 0.881 h_{s}$, although these differences are often ignored.
} 
increasing scale height with increasing Galactocentric radius, with a pronounced flaring at radii $\gtrsim 10 \mathrm{kpc}$. Based on the CO observations of Nakanishi \& Sofue (2006), we parameterise this dependence of scale height (in pc) on $R$ by a quadratic function:

$$
H=32+1.6 \times 10^{-3} R+4.0 \times 10^{-7} R^{2}
$$

where $R$ is in pc. The scale height is then $K_{i} H$, where $K_{i}$ is a constant factor for the $i$ th component.

We model the thin disk with $\operatorname{sech}^{2}(x)$ functions for both the radial and $z$ variations as follows:

$$
n_{2}=n_{2_{0}} g_{d} \operatorname{sech}^{2}\left(\frac{R-B_{2}}{A_{2}}\right) \operatorname{sech}^{2}\left(\frac{z-z_{w}}{K_{2} H}\right)
$$

where $n_{2_{0}}$ is mid-plane electron density at the ring central radius $R=B_{2}, g_{d}$ is defined in $\S 3.1$. We fix $A_{2}=1200 \mathrm{pc}$ and $B_{2}=4000 \mathrm{pc}$ and fit for $n_{2_{0}}$ and $K_{2}$. Since the Galactic tracers basically determine or are determined by the distribution of $n_{e}^{2}$, we expect $K_{2} \sim 2$.

\subsection{Spiral arms}

Outside the molecular ring, the distribution of free electrons in the Galactic disk is dominated by spiral structure. This structure was first identified in our Galaxy with observations of HI (Oort et al. 1958) and was first clearly delineated by Georgelin \& Georgelin (1976) using kinematic distances of HII regions supplemented by optical observations of massive stars. Many other tracers such as CO emission, especially from giant molecular clouds (e.g. Grabelsky et al. 1988) and methanol masers (e.g. Green et al. 2011) have helped to define the spiral structure. The importance of spiral structure for models of the Galactic $n_{e}$ distribution was first indicated (Johnston et al. 1992; Taylor \& Cordes 1993) by the asymmetry in the distribution of pulsar DMs between the first (northern) and fourth (southern) Galactic quadrants as illustrated in Figure 1.

In a recent study, Hou \& Han (2014) updated the catalogs of spiral arm tracers, including more than 2500 HII regions, 1300 giant molecular clouds, and $9006.7-\mathrm{GHz}$ methanol masers. They used these data to investigate the spiral structure of the Galaxy, assuming a logarithmic spiral form:

$$
\ln \left(\frac{R}{R_{a_{i}}}\right)=\left(\phi-\phi_{a_{i}}\right) \tan \psi_{a_{i}}
$$

where $R_{a_{i}}, \phi_{a_{i}}$ and $\psi_{a_{i}}$ are the initial radius, the start azimuth angle and the pitch angle for the $i$ th arm. As the HII-region data have the most complete and reliable distance estimates and four-armed spirals are preferred, we have adopted the corresponding Hou \& Han (2014) fit with solar galactocentric radius $R_{\odot}=8.3 \mathrm{kpc}$ and circular velocity

for the Local Standard of Rest of $239 \mathrm{~km} \mathrm{~s}^{-1}$ to define the spiral structure in our model (apart from a minor

\begin{tabular}{|c|c|c|c|c|}
\hline Arm name & Index & $\begin{array}{c}R_{a_{i}} \\
(\mathrm{kpc})\end{array}$ & $\begin{array}{l}\phi_{a_{i}} \\
\left(^{\circ}\right)\end{array}$ & $\begin{array}{c}\psi_{a_{i}} \\
\left(^{\circ}\right)\end{array}$ \\
\hline Norma - Outer & 1 & 3.35 & 44.4 & 11.43 \\
\hline Perseus & 2 & 3.71 & 120.0 & 9.84 \\
\hline Carina - Sagittarius & 3 & 3.56 & 218.6 & 10.38 \\
\hline Crux - Scutum & 4 & 3.67 & 330.3 & 10.54 \\
\hline Local & 5 & 8.21 & 55.1 & 2.77 \\
\hline
\end{tabular}
modification to the start position of the Perseus arm). The adopted arm parameters are given in Table 1.

Table 1. Adopted spiral-arm parameters

We adopt a $\operatorname{sech}^{2}\left(s_{a}\right)$ cross-section for the electron density in the arms, where $s_{a}$ is the perpendicular distance to the arm axis in the $x-y$ plane. We also adopt a $\operatorname{sech}^{2}(x)$ dependence for the radial variation in $n_{e}$, joining with the thin disk at $R=B_{2}$. The radial dependence of scale height defined by Equation 8 is assumed for all arms. Although we solve for the spiral-arm scale factor $K_{a}$, an indication of its size can be obtained from the $z$-dependence of $\mathrm{H} \alpha$ intensity shown by Hill et al. (2014). This suggests a FWHM for the Scutum arm HII-region component at $R \sim 5 \mathrm{kpc}$ of about $170 \mathrm{pc}$, corresponding to a $\operatorname{sech}^{2}(x)$ scale height for $n_{e}^{2} \sim 95 \mathrm{pc}$. Hence, the $n_{e}$ scale height $H_{a_{4}} \sim 190 \mathrm{pc}$, 
corresponding to $K_{a} \sim 3.8$. Although the spiral-arm $n_{e}$ is dominated by individual HII regions, except for a few relatively local features, we assume that these average to a uniform arm density over the typically long paths to pulsars.

The electron density contributed by the spiral arms is therefore defined as follows:

$$
n_{a}=\sum_{i=1}^{n} g_{d} n_{a_{i}} \operatorname{sech}^{2}\left(\frac{s_{a_{i}}}{w_{a_{i}}}\right) \operatorname{sech}^{2}\left(\frac{R-B_{2}}{A_{a}}\right) \operatorname{sech}^{2}\left(\frac{z-z_{w}}{K_{a} H}\right)
$$

where, for arm $i, n_{a_{i}}$ is the mid-plane density at $R=B_{2}$. The electron densities $n_{a_{i}}$ refer to the point were the arms join the thin disk, at $B_{2}=4.0 \mathrm{kpc}$. The arms are assumed to have the same radial termination $g_{d}$ as the thick disk (Equation 6). Where the thin disk and an arm overlap, the larger of the two densities $n_{2}$ and $n_{a_{i}}$ is taken. Because of covariance with the arm electron densities $n_{a_{i}}$, the widths of the arms, $w_{a_{i}}$ were held fixed in the final fit at values determined from preliminary global fits.

Preliminary model fits showed consistently over-estimated model distances for pulsars in and beyond the Carina tangential zone $\left(l \sim 285^{\circ}-300^{\circ}\right)$ and, conversely, consistently under-estimated model distances for pulsars in and beyond the Sagittarius tangential region $\left(l \sim 45^{\circ}-55^{\circ}\right)$. This asymmetry is also visible in Figure 1 which shows that DMs in the Sagittarius region are lower than those in the Carina region. To overcome these problems, we have defined an over-dense region in the Carina arm and an underdense region in Sagittarius, modifying the density of spiral arm 3, as follows:

$$
n_{a_{3}}^{\prime}= \begin{cases}n_{a_{3}}\left\{1+n_{\mathrm{CN}} \exp \left[-\left(\frac{\phi-\phi_{\mathrm{CN}}}{\Delta \phi_{\mathrm{CN}}}\right)^{2}\right]\right\}\left\{1-n_{\mathrm{SG}} \exp \left[-\left(\frac{\phi-\phi_{\mathrm{SG}}}{\Delta \phi_{\mathrm{SG}}}\right)^{2}\right]\right\}, & \phi<\phi_{\mathrm{CN}} \\ n_{a_{3}}\left\{1+n_{\mathrm{CN}}\right\}\left\{1-n_{\mathrm{SG}} \exp \left[-\left(\frac{\phi-\phi_{\mathrm{SG}}}{\Delta \phi_{\mathrm{SG}}}\right)^{2}\right]\right\}, & \phi>\phi_{\mathrm{CN}}\end{cases}
$$

where $n_{\mathrm{CN}}$ and $n_{\mathrm{SG}}$ represent the over-density and under-density of the Carina arm and Sagittarius region, respectively, and $\phi_{\mathrm{CN}}, \Delta \phi_{\mathrm{CN}}$ and $\phi_{\mathrm{SG}}, \Delta \phi_{\mathrm{SG}}$ are the central azimuth and half-width in azimuth of the low- $\phi$ side of the Carina arm and the Sagittarius under-density, respectively.

\subsection{Galactic Center}

The Galactic Center region is unique in the Galaxy because of the $4 \times 10^{6} \mathrm{M}_{\odot}$ black hole, seen as the point source Sgr A*, at its center, its high gas density, high star-formation rate, high magnetic fields and numerous other energetic phenomena occuring in the region (see Genzel et al. 2010, for a review). These phenomena lead to a high density of ionised gas which will affect signals from any pulsar in or behind the region. In the ATNF Pulsar Catalogue (V1.54), there are eight known pulsars that have $|l|<1^{\circ}$ and $|b|<0.25^{\circ}$. All eight have DMs $\gtrsim 1000 \mathrm{~cm}-3 \mathrm{pc}$ and could plausibly lie close to the Galactic Center. However, only for the closest one, PSR J1745-2900 which is only 2!! 4 from Sgr A* (Bower et al. 2015), has a case been made for a physical association (Rea et al. 2013; Bower et al. 2015). As well as having the highest DM of the eight, it also has a very large rotation measure (Shannon \& Johnston 2013; Eatough et al. 2013), strengthening the case for a physical association. With only one pulsar, we cannot constrain the form or extent of the ionised gas from the pulsar observations. However, we can use observations of molecular gas (e.g. Oka et al. 2012), radio thermal continuum emission (e.g. Law et al. 2008) and radio recombination lines (Law et al. 2009; Alves et al. 2015) to establish a sufficiently reliable definition of the region as follows:

$$
\begin{gathered}
R_{\mathrm{GC}}=\left[\left(x-x_{\mathrm{GC}}\right)^{2}+\left(y-y_{\mathrm{GC}}\right)^{2}\right]^{1 / 2} \\
n_{\mathrm{GC}}=n_{\mathrm{GC}_{0}} \exp \left[-\left(\frac{R_{\mathrm{GC}}}{A_{\mathrm{GC}}}\right)^{2}\right] \operatorname{sech}^{2}\left(\frac{z-z_{\mathrm{GC}}}{H_{\mathrm{GC}}}\right)
\end{gathered}
$$

where $\left(x_{\mathrm{GC}}, y_{\mathrm{GC}}, z_{\mathrm{GC}}\right)=(+50 \mathrm{pc}, 0 \mathrm{pc},-7 \mathrm{pc})$ is the center of the disk, $R_{\mathrm{GC}}$ is the radial distance from the center of the disk in the $x-y$ plane, $A_{\mathrm{GC}}$ is its radial scale length and the $H_{\mathrm{GC}}$ is its scale height. We fix $A_{\mathrm{GC}}=160 \mathrm{pc}$ and $H_{\mathrm{GC}}=35 \mathrm{pc}$, based largely on the CO observations of Oka et al. (2012).

\subsection{Gum Nebula}

The Gum Nebula is the largest known optical emission nebula in the southern sky and contributes significantly to the DM of pulsars within and behind it. All pulsar-based $n_{e}$ models since Manchester \& Taylor (1981) have included the Gum Nebula as a component. On the basis of an image from the Southern H $\alpha$ Sky Survey Atlas, (Finkbeiner 
2003), Purcell et al. (2015) modelled the Gum Nebula with a spherical shell of outer angular radius $\sim 23^{\circ}$ as seen from the Sun (corresponding to a physical radius of about $170 \mathrm{pc}$ ) and thickness about 20 pc. Based on the DMs of pulsars in the region and the $\mathrm{H} \alpha$ image (Purcell et al. 2015), we adopt a modified version of the Purcell et al. (2015) model with an ellipsoidal shell, extended in the $z$-direction, and centered at $l=264^{\circ}, b=-4^{\circ}$ at distance 450 pc:

$$
\left(\frac{x-x_{\mathrm{GN}}}{A_{\mathrm{GN}}}\right)^{2}+\left(\frac{y-y_{\mathrm{GN}}}{A_{\mathrm{GN}}}\right)^{2}+\left(\frac{z-z_{\mathrm{GN}}}{K_{\mathrm{GN}} A_{\mathrm{GN}}}\right)^{2}=1
$$

where $\left(x_{\mathrm{GN}}, y_{\mathrm{GN}}, z_{\mathrm{GN}}\right)=\left(-446 \mathrm{pc}, R_{\odot}+47 \mathrm{pc},-31 \mathrm{pc}\right)$ is the center of the shell, $K_{\mathrm{GN}}$ is the ratio of the $z$-axis dimension to that in the $x-y$ plane and $A_{\mathrm{GN}}$ is the mid-line radius of the shell in the $x-y$ plane. The shell electron density is assumed to have a gaussian profile with $1 / \mathrm{e}$ half-width $W_{\mathrm{GN}}$ :

$$
n_{\mathrm{GN}}=n_{\mathrm{GN}_{0}} \exp \left[-\left(\frac{s_{\mathrm{GN}}}{W_{\mathrm{GN}}}\right)^{2}\right]
$$

where $s_{\mathrm{GN}}$ is the perpendicular distance to the mid-point of the ellipsoidal shell. Eight pulsars with model-independent distances and known DMs are affected by the Gum Nebula. The value of $K_{\mathrm{GN}}$ was fixed at 1.4 in the global parameter fit.

\subsection{Local Bubble region}

Several tracers of the local ISM indicate that the Sun resides in a relatively low-density cavity often called the Local Bubble. Low densities within 100 pc or so of the Sun are indicated by low interstellar reddening of nearby stars (Reis et al. 2011; Lallement et al. 2014), HI 21-cm spectral-line observations (see Snowden et al. 2015), NaI and CaII absorption lines in the spectra of nearby early-type stars (e.g., Welsh et al. 2010) and observations of the "diffuse interstellar bands" (Bailey et al. 2016). A background of diffuse soft X-ray emission (Snowden et al. 1995) is generally attributed to hot $\left(10^{6} \mathrm{~K}\right)$ gas in the Local Bubble (Puspitarini et al. 2014; Snowden et al. 2015) although this remains somewhat controversial with X-ray emission from solar-wind charge-exchange interactions possibly contributing to the background (see Reis et al. 2011). The stellar absorption-line observations of Welsh et al. (2010) also indicate the presence of partially ionised "cloudlets" of lower temperature gas within the Local Bubble as well as clouds of colder and denser gas around its boundary that would be expected to have ionised outer layers. Even if much of the volume of the Local Bubble is occupied by high-temperature low-density gas, these ionised lower-temperature regions will contribute to pulsar dispersion within the bubble.

Observations of NaI absorption in nearby stars with accurate distances, either from EUV observations (Welsh et al. 1999) or Hipparcos parallaxes (Lallement et al. 2003), led to a model for the Local Bubble consisting of a tilted chimney-like cavity extending out of the Galactic disk on both sides and bounded by regions of high-density gas within the disk. We have implemented this model with a cylinder of radius $R_{\mathrm{LB}}=110 \mathrm{pc}$, tilted at $20^{\circ}$ toward the Galactic anticentre (i.e., in the $+y$ direction) above the plane and centered $40 \mathrm{pc}$ from the Sun in the same direction, together with two regions of enhanced electron density on its boundary. The radial distance from the cylinder axis is given by:

$$
r_{\mathrm{LB}}=\left\{\left[0.94\left(y-R_{\odot}-40\right)-0.34 z\right]^{2}+x^{2}\right\}^{1 / 2}
$$

and the electron density within the cylinder (i.e., $r_{\mathrm{LB}}<R_{\mathrm{LB}}$ and $\left.\left[n_{1}+\max \left(\mathrm{n}_{2}, \mathrm{n}_{\mathrm{a}}\right)\right]>\left[\mathrm{n}_{\mathrm{LB} 1}+\mathrm{n}_{\mathrm{LB} 2}\right]\right)$ is:

$$
n_{\mathrm{LB}}=J_{\mathrm{LB}} n_{1}+\max \left(n_{2}, n_{a}\right)
$$

where $n_{1}$ is the electron density of the thick disk, $n_{a}$ is the summed density of spiral arms (Equation 11$)$ and $J_{\mathrm{LB}}$ is a scale factor similar to $J_{\mathrm{FB}}(\S 3.1)$. The two regions of enhanced density on the boundary of the Local Bubble, LB1 and LB2, are defined by

$$
\begin{aligned}
& n_{\mathrm{LB} 1}=n_{\mathrm{LB} 1_{0}} \operatorname{sech}^{2}\left(\frac{l-l_{\mathrm{LB} 1}}{\Delta l_{\mathrm{LB} 1}}\right) \operatorname{sech}^{2}\left(\frac{r_{\mathrm{LB}}-R_{\mathrm{LB}}}{W_{\mathrm{LB} 1}}\right) \operatorname{sech}^{2}\left(\frac{z}{H_{\mathrm{LB} 1}}\right) \\
& n_{\mathrm{LB} 2}=n_{\mathrm{LB} 2_{0}} \operatorname{sech}^{2}\left(\frac{l-l_{\mathrm{LB} 2}}{\Delta l_{\mathrm{LB} 2}}\right) \operatorname{sech}^{2}\left(\frac{r_{\mathrm{LB}}-R_{\mathrm{LB}}}{W_{\mathrm{LB} 2}}\right) \operatorname{sech}^{2}\left(\frac{z}{H_{\mathrm{LB} 2}}\right) .
\end{aligned}
$$

Although guided by the stellar absorption-line results, the central longitudes and angular widths of LB1 and LB2 are fitted for in the global model (along with the respective densities) and hence are primarily determined by the DMs of relatively local pulsars. 


\subsection{Loop $I$}

After fitting for the model components described above, we noticed that a number of relatively nearby pulsars in the region $0^{\circ} \lesssim l \lesssim 30^{\circ}$ and $b \gtrsim 0^{\circ}$ had over-estimated distances. This region roughly defines the location of the North Polar Spur, apparently the brightest feature of the bubble known as Loop I (Berkhuijsen et al. 1971). Although not universally accepted (see, e.g., Bland-Hawthorn \& Cohen 2003; Puspitarini et al. 2014), we adopt the view that the North Polar Spur is associated with Loop I and that Loop I is relatively local, probably associated with outflow from the Scorpio - Centaurus OB association (see, e.g., Wolleben 2007, and references therein). Following Wolleben (2007), we model Loop I as a spherical shell centered $200 \mathrm{pc}$ from the Sun in the direction $l=346^{\circ}, b=3^{\circ}$ and the ionisation as a spherical cap centred in the north-eastern part of the shell. The defining equations for the electron density at a point $\left(r_{\mathrm{LI}}, \theta_{\mathrm{LI}}\right)$, where $r_{\mathrm{LI}}$ is the radial distance from the center of the shell and $\theta_{\mathrm{LI}}$ is the angle to the $+x$ axis, are therefore:

$$
\begin{gathered}
n_{\mathrm{LI}}=n_{\mathrm{LI}_{0}} \exp \left[-\left(\frac{r_{\mathrm{LI}}-R_{\mathrm{LI}}}{W_{\mathrm{LI}}}\right)^{2}\right] \exp \left[-\left(\frac{\theta_{\mathrm{LI}}}{\Delta \theta_{\mathrm{LI}}}\right)^{2}\right] \\
r_{\mathrm{LI}}=\left[\left(x-x_{\mathrm{LI}}\right)^{2}+\left(y-y_{\mathrm{LI}}\right)^{2}+\left(z-z_{\mathrm{LI}}\right)^{2}\right]^{1 / 2} \\
\theta=\arccos \left[\frac{\left(x-x_{\mathrm{LI}}\right) \cos \theta_{\mathrm{LI}}+\left(z-z_{\mathrm{LI}}\right) \sin \theta_{\mathrm{LI}}}{r_{\mathrm{LI}}}\right]
\end{gathered}
$$

where $R_{\mathrm{LI}}$ is the shell mid-line radius, $W_{\mathrm{LI}}$ is the shell half-thickness, $\theta_{\mathrm{LI}}$ is the angle between the direction of the center of the spherical cap and the $+x$ direction, $\Delta \theta_{\mathrm{LI}}$ is the extent of the cap in $\theta_{\mathrm{LI}},\left(x_{\mathrm{LI}}, y_{\mathrm{LI}}, z_{\mathrm{LI}}\right)=(-48 \mathrm{pc}, 8106 \mathrm{pc}, 10 \mathrm{pc})$ is the center of the shell.

\subsection{Total Galactic electron density}

Given the above equations defining each component of the Galactic electron density, the $n_{e}$ at any $(x, y, z)$ within the Galaxy is defined to be:

$$
n_{\mathrm{Gal}}=\left\{1-w_{\mathrm{LB}}\right\}\left\{\left[1-w_{\mathrm{GN}}\right]\left[\left(1-w_{\mathrm{LI}}\right)\left(n_{0}+n_{\mathrm{GC}}\right)+w_{\mathrm{LI}} n_{\mathrm{LI}}\right]+w_{\mathrm{GN}} n_{\mathrm{GN}}\right\}+w_{\mathrm{LB}}\left(n_{\mathrm{LB} 1}+n_{\mathrm{LB} 2}\right)
$$

where

$$
n_{0}=n_{1}+\max \left(n_{2}, n_{a}\right)
$$

except that within the Fermi Bubbles (Equation 7)

$$
n_{0}=J_{\mathrm{FB}} n_{1}+\max \left(n_{2}, n_{a}\right) .
$$

Within the Local Bubble, i.e., $r_{\mathrm{LB}}<R_{\mathrm{LB}}=110$ pc (Equation 17),

$$
n_{0}=J_{\mathrm{LB}} n_{1}+\max \left(n_{2}, n_{a}\right)
$$

and, if $\left(n_{\mathrm{LB} 1}+n_{\mathrm{LB} 2}\right)>n_{0}, w_{\mathrm{LB}}=1$, otherwise $w_{\mathrm{LB}}=0$. Outside the Local Bubble, if $\left(n_{\mathrm{LB} 1}+n_{\mathrm{LB} 2}\right)>n_{0}$ and $\left(n_{\mathrm{LB} 1}+n_{\mathrm{LB} 2}\right)>n_{\mathrm{GN}}, w_{\mathrm{LB}}=1$, otherwise $w_{\mathrm{LB}}=0$. For Loop I, if $n_{L I}>n_{0}, w_{\mathrm{LI}}=1$, otherwise $w_{\mathrm{LI}}=0$, and similarly for the Gum Nebula.

In total, the Galactic model has 96 parameters, of which 64 are fixed and 32 are fitted.

\subsection{The Magellanic Clouds}

Since the known extra-galactic pulsars are all associated with the Magellanic Clouds, we include these Clouds in our electron-density model. Following van der Marel \& Cioni (2001) and Haschke et al. (2012a), we model the LMC as a thick inclined disk with inclination angle $i=32^{\circ}$ and line of nodes at astronomical position angle $\Theta=116^{\circ}$. It is convenient to define coordinate systems $\left(x_{c}, y_{c}, z_{c}\right)$ with $x_{c}, y_{c}$ in the plane of the sky, $x_{c}$ in the direction of decreasing right ascension (toward west), $y_{c}$ toward north and $z_{c}$ toward the observer, and $\left(x^{\prime}, y^{\prime}, z^{\prime}\right)$, with $x^{\prime}$ along the line of nodes at an angle $\theta=\Theta+90^{\circ}$ to the $x_{c}$ axis, $y^{\prime}$ in the plane of the galaxy on the far side and $z^{\prime}$ normal to the galaxy plane toward the observer. Both coordinate systems have their origin at the center of the LMC, which we define to lie in the direction $\left(\alpha_{\mathrm{LMC}}, \delta_{\mathrm{LMC}}\right)=\left(05^{\mathrm{h}} 24^{\mathrm{m}},-69^{\circ} 45^{\prime}\right)$ at distance $D_{\mathrm{LMC}}=49.7 \mathrm{kpc}$ (Walker 2012). Relations for converting between Galactic $(l, b, D)$, Galactic $(x, y, z)$, the celestial $\left(x_{c}, y_{c}, z_{c}\right)$ system and the $\left(x^{\prime}, y^{\prime}, z^{\prime}\right)$ system are given in Appendix C. 
We model the electron density of the LMC, $n_{\mathrm{LMC}}$, as a thick disk centered at the origin of the $\left(x^{\prime}, y^{\prime}, z^{\prime}\right)$ frame, together with an additional component, $n_{30 \mathrm{D}}$, representing the giant HII complex 30 Doradus:

$$
\begin{gathered}
R_{\mathrm{LMC}}=\left(x^{\prime 2}+y^{\prime 2}\right)^{1 / 2} \\
n_{\mathrm{LMC}}=n_{\mathrm{LMC}_{0}} \exp \left[-\left(\frac{R_{\mathrm{LMC}}}{A_{\mathrm{LMC}}}\right)^{2}\right] \operatorname{sech}^{2}\left(\frac{z^{\prime}}{H_{\mathrm{LMC}}}\right)
\end{gathered}
$$

where $A_{\mathrm{LMC}}$ is the radial scale of the disk, $H_{\mathrm{LMC}}$ is its scale height.

The giant HII region 30 Doradus is a major feature of the LMC and warrants separate treatment. It is almost certainly responsible for the anomalously high DM of PSR J0537-69 (Table A6). As seen in radio continuum (Hughes et al. 2007 ) and $\mathrm{H} \alpha$ (Gaustad et al. 2001), 30 Doradus is not far from the nominal center of the LMC at $\left(\alpha_{30 \mathrm{D}}, \delta_{30 \mathrm{D}}\right)=$ $\left(05^{\mathrm{h}} 40^{\mathrm{m}},-69^{\circ} 00^{\prime}\right)$ and it is believed to be at a similar distance. We model its electron density as a spherical Gaussian distribution:

$$
\begin{gathered}
R_{30 \mathrm{D}}=\left[\left(x^{\prime}-x_{30 \mathrm{D}}^{\prime}\right)^{2}+\left(y^{\prime}-y_{30 \mathrm{D}}^{\prime}\right)^{2}+z^{\prime 2}\right]^{1 / 2} \\
n_{30 \mathrm{D}}=n_{30 \mathrm{D}_{0}} g_{30 \mathrm{D}} \exp \left[-\left(\frac{R_{30 \mathrm{D}}}{A_{30 \mathrm{D}}}\right)^{2}\right]
\end{gathered}
$$

where $A_{30 \mathrm{D}}$ is the radial scale length. Note that we have placed the center of 30 Doradus in the plane of the LMC, i.e., $z_{30 \mathrm{D}}^{\prime}=0$.

We fix $A_{\mathrm{LMC}}=3000 \mathrm{pc}$ and $H_{\mathrm{LMC}}=800 \mathrm{pc}$, based largely on the distribution of the Cepheid variables in the LMC, which represent the younger stellar population (Haschke et al. 2012a), and fit for $n_{\mathrm{LMC}_{0}}$ based on the LMC pulsars with known DM. Similarly we fix $A_{30 \mathrm{D}}=450 \mathrm{pc}$ based on the radio continuum size and fit for $n_{30 \mathrm{D}_{0}}$.

Compared to the LMC, the SMC has a much greater depth and, while there is some evidence for a flattened distribution of young stars (e.g., Haschke et al. 2012b), the parameters are relatively uncertain. Because of this and the relatively small number of associated radio pulsars, we have chosen a simple model for the SMC, a spherical Gaussian nebula centered at $\left(\alpha_{\mathrm{SMC}}, \delta_{\mathrm{SMC}}\right)=\left(00^{\mathrm{h}} 51^{\mathrm{m}},-73^{\circ} 06^{\prime}\right)$ at distance $D_{\mathrm{SMC}}=59.7 \mathrm{kpc}$ :

$$
\begin{gathered}
R_{\mathrm{SMC}}=\left[\left(x-x_{\mathrm{SMC}}\right)^{2}+\left(y-y_{\mathrm{SMC}}\right)^{2}+\left(z-z_{\mathrm{SMC}}\right)^{2}\right]^{1 / 2} \\
n_{\mathrm{SMC}}=n_{\mathrm{SMC}_{0}} \exp \left[-\left(\frac{R_{\mathrm{SMC}}}{A_{\mathrm{SMC}}}\right)^{2}\right]
\end{gathered}
$$

where $A_{\mathrm{SMC}}$ is the radial scale length. We fix $A_{\mathrm{SMC}}=3000 \mathrm{pc}$ based on the distribution of Cepheid variables (Haschke et al. 2012b) and fit for $n_{\mathrm{SMC}_{0}}$.

In total, the Magellanic Cloud model has 18 parameters of which 15 are fixed and three are fitted.

\subsection{Intergalactic medium}

In order to provide a convenient method for estimating the redshifts and distances of current, future and simulated FRBs from their DM (and vice versa), YMW16 includes a model for the free electron density in the intergalactic medium. Following Katz (2016), in the approximation of zero curvature, the comoving distance $D$ to an FRB is given by:

$$
D=\frac{c}{H_{0}} \ln (1+z)
$$

where $H_{0}$ is the Hubble Constant and the redshift $z$ is given by:

$$
z=\frac{\mathrm{DM}_{\mathrm{IGM}} \mathrm{H}_{0}}{c n_{\mathrm{IGM}}}=\frac{\left[\mathrm{DM}-\left(\mathrm{DM}_{\mathrm{Gal}}+\mathrm{DM}_{\mathrm{MC}}+\mathrm{DM}_{\mathrm{Host}}\right)\right] H_{0}}{c n_{\mathrm{IGM}}}
$$

where DM is the observed FRB dispersion measure, $\mathrm{DM}_{\mathrm{Gal}}$ is the total Galactic DM along the path to the FRB, $\mathrm{DM}_{\mathrm{MC}}$ is any Magellanic Cloud contribution, $\mathrm{DM}_{\mathrm{IGM}}$ is the contribution from the IGM and $\mathrm{DM}_{\text {Host }}$ is the contribution of the FRB host galaxy to the observed DM. We adopt a value for $H_{0}$ of $67.3 \mathrm{~km} \mathrm{~s}^{-1} \mathrm{Mpc}^{-1}$ (Planck Collaboration 2014) and adopt the Katz (2016) value for $n_{\mathrm{IGM}}, 0.16 \mathrm{~m}^{-3}$. 
We know little about the host galaxies of FRBs and so it is difficult to estimate $\mathrm{DM}_{\text {Host }}$. Furthermore, the observed dispersive delay is a factor $(1+z)$ larger than the delay at the host galaxy, where $z$ is the host galaxy redshift, and the radio frequency at the host galaxy is a factor of $(1+z)$ larger than the observed radio frequency. The net effect of these two factors is that, in the host galaxy frame, $\mathrm{DM}_{\text {Host }, \mathrm{z}}=(1+z) \mathrm{DM}_{\text {Host }}$. Lorimer et al. (2007) assumed a $\mathrm{DM}_{\text {Host }}$ of $200 \mathrm{~cm}^{-3} \mathrm{pc}$ in their analysis of the original FRB010724, but DM contributions from elliptical galaxies or randomly oriented spirals are likely to be less than that, especially given the time dilation factor. Following Thornton et al. (2013) and Caleb et al. (2016) we adopt $\mathrm{DM}_{\text {Host }}=100 \mathrm{~cm}^{-3} \mathrm{pc}$ as the default value, but allow the optional entry of a different value.

To avoid pulsars with DMs that are larger than the maximum YMW16 model prediction in that direction being placed at (obviously incorrect) cosmological distances, Equations 34 and 35 are invoked only if an "IGM" input flag is set in the DM $-D$ routine. Otherwise, reasonable upper bounds are placed on the model distance.

\subsection{Interstellar and intergalactic scattering}

As discussed in $\S 1$, we do not include interstellar scattering results as an input to the YMW16 model. However, it is useful to give an estimate of scattering timescales $\left(\tau_{\mathrm{sc}}\right)$ as part of the output of the model. In the absence of reliable data about the distribution and strength of scattering regions in the Galaxy, we adopt a simple approach to this, using the relation obtained by Krishnakumar et al. (2015) for the DM dependence of observed $\tau_{\mathrm{sc}}$ values (in units of seconds and scaled to $1 \mathrm{GHz}$ assuming $\left.\tau_{\mathrm{sc}} \propto \nu^{-4.0}\right)$ :

$$
\tau_{\mathrm{sc}}=4.1 \times 10^{-11} \mathrm{DM}^{2.2}\left(1.0+0.00194 \mathrm{DM}^{2.0}\right) .
$$

Observed values of $\tau_{\text {sc }}$ have an rms scatter about the fitted line of close to an order of magnitude, but the variation of $\tau_{\mathrm{sc}}$ over the range of observed DMs is about eight orders of magnitude, so it is a useful predictor.

For the Magellanic Clouds, scattering will occur in both the Galaxy and the Clouds. (Dispersion and scattering in the intervening IGM is negligible.) Equation 36 estimates the scatter-broadening for a pulsar having a given DM. On average, the scattering screen will be about half-way between the Sun and the pulsar. For a pulsar in the Magellanic Clouds, the wave incident on the Galaxy is essentially plane. Therefore, the Galactic component of the scattering delay is just half of the $\tau_{\mathrm{sc}}$ computed from Equation 36 with $\mathrm{DM}=\mathrm{DM}_{\mathrm{Gal}}$, again assuming that the scattering screen is halfway along the Galactic path (Rickett 1990), that is, $\tau_{\text {Gal }}=0.5 \tau_{\mathrm{sc}}\left(\mathrm{DM}_{\mathrm{Gal}}\right)$. If we assume that the scattering properties of the Magellanic Clouds are roughly the same as those of our Galaxy, we can apply these considerations to the Clouds as well, just reversing the direction of propagation. That is, $\tau_{\mathrm{MC}}=0.5 \tau_{\mathrm{sc}}\left(\mathrm{DM}_{\mathrm{MC}}\right)$. Since the two scattering distributions are statistically independent, the net effect is given by the convolution of the two scattering functions. This convolved distribution is given by

$$
f(t)=\frac{1}{\tau_{\text {Gal }}} \exp \left(-t / \tau_{\text {Gal }}\right) * \frac{1}{\tau_{\mathrm{MC}}} \exp \left(-t / \tau_{\mathrm{MC}}\right)
$$

where $\tau_{\text {Gal }}=0.5 \tau_{\mathrm{sc}}$ from Equation 36 with $\mathrm{DM}=\mathrm{DM}_{\mathrm{Gal}}$, and similarly for $\tau_{\mathrm{MC}}$ with $\mathrm{DM}_{\mathrm{MC}}$. The convolution integral for $t \geq 0$ is

$$
f(t)=\frac{1}{\tau_{\mathrm{Gal}} \tau_{\mathrm{MC}}} \int_{0}^{t} d t^{\prime} \exp \left[-\left(t-t^{\prime}\right) / \tau_{\mathrm{Gal}}\right] \exp \left[-t^{\prime} / \tau_{\mathrm{MC}}\right]
$$

and hence

$$
f(t)=\frac{1}{\left(\tau_{\mathrm{MC}}-\tau_{\mathrm{Gal}}\right)}\left[\exp \left(-t / \tau_{\mathrm{MC}}\right)-\exp \left(-t / \tau_{\mathrm{Gal}}\right)\right] .
$$

In the limit of $\tau_{\mathrm{Gal}}=\tau_{\mathrm{MC}}=\tau$, this reduces to

$$
f(t)=\frac{t}{\tau^{2}} \exp (-t / \tau)
$$

(cf., Williamson 1972). Equation 39 shows that, for short times, the rise of $f(t)$ is determined by the inverted decay of the term with the shorter scattering time whereas, at longer times, the decay of $f(t)$ is dominated by the longer scattering time (or by the scattering time of each screen if they are equal). This longer timescale will determine measured values of FRB scattering times. We therefore model the scattering of Magellanic Cloud pulsars using

$$
\tau_{\mathrm{sc}}(\mathrm{MC})=\max \left(\tau_{\mathrm{Gal}}, \tau_{\mathrm{MC}}\right)
$$

Scattering timescales have been measured for ten of the 17 known FRBs, with upper limits for the remaining seven (Table A7). The origin of this broadening is a matter of considerable debate. With the possible exceptions of the 
three known FRBs at low Galactic latitude (Table A7), scattering in our Galaxy (or the SMC for FRB010724) is too small to account for the observed $\tau_{\mathrm{sc}}$. This leaves the IGM and the host galaxy as possible sources of the observed scattering.

For the host galaxy, scattering could arise in the ISM along the path (see, e.g., Xu \& Zhang 2016 ; Cordes et al. 2016) or in the immediate environs of the FRB source (see, e.g., Masui et al. 2015). Because of the extremely small lever-arm factor $a(1-a)$, where the screen is at $a D$ and $D$ is the distance to the source (cf., Williamson 1972), origin of the observed scattering in the FRB environs would require extremely large scattering angles. While this is not impossible, it requires most (but not all) FRB sources to lie in very dense and turbulent nebulae. This is exacerbated by time dilation of the observed scatter broadening. Similar to the effect on DM as discussed above, the observed scatter broadening is a factor $(1+z)$ larger than the broadening in the host frame and the radio frequency at host galaxy is a factor of $(1+z)$ larger than the observed radio frequency. Since scatter broadening scales approximately as $\nu^{-4.0}$ the observed scattering time is reduced by a factor $\sim(1+z)^{3}$ (cf., Macquart \& Koay 2013). In view of these factors, we believe that FRB scattering is unlikely to arise in the immediate environs of the FRB source.

Scattering in the ISM of the host galaxy can be modelled in a similar way to scattering in our Galaxy, that is, based on the $\tau_{\mathrm{sc}}-\mathrm{DM}$ relation (Equation 36). This scatter broadening is also reduced by the $\sim(1+z)^{3}$ factor, but there is a significant additional factor. As discussed above in $\S 3.10$, the DM in the host galaxy frame is a factor $(1+z)$ larger than $\mathrm{DM}_{\text {Host }}$, the observed DM contribution attributed to the host galaxy. Following the same logic as for scattering in the Magellanic Clouds and using Equation 36 in the frame of the host galaxy, we adopt $\tau_{\text {Host }, z}=0.5 \tau_{\mathrm{sc}}[(1+z) \mathrm{DM}$ Host $]$. Since $\tau_{\text {sc }}$ is roughly proportional to $\mathrm{DM}^{2.2}$ (Equation 36), the net effect of time dilation is that the observed scatter broadening due to the host galaxy $\tau_{\text {Host }} \sim \tau_{\text {Host }, z} /(1+z)$. Consequently, even for large star-forming galaxies similar to our own Galaxy, the host-galaxy ISM is unlikely to contribute significantly to the observed FRB scattering.

The remaining location for FRB scattering is the IGM between us and the host galaxy. Luan \& Goldreich (2014) have argued that the level of turbulence in the IGM is too small to account for the observed scattering delays. On the other hand, Macquart \& Koay (2013) argue that relatively dense intracluster gas or individual galaxies along the path to the FRB could account for the observed scattering. For known FRBs with estimated $z \lesssim 2$, the expected number of such intervening screens is low, $\lesssim 2$. This small number of effective screens provides a natural explanation for the large variation in observed scattering times. FRBs with only upper limits on scatter broadening simply have no strong scattering screen along the path.

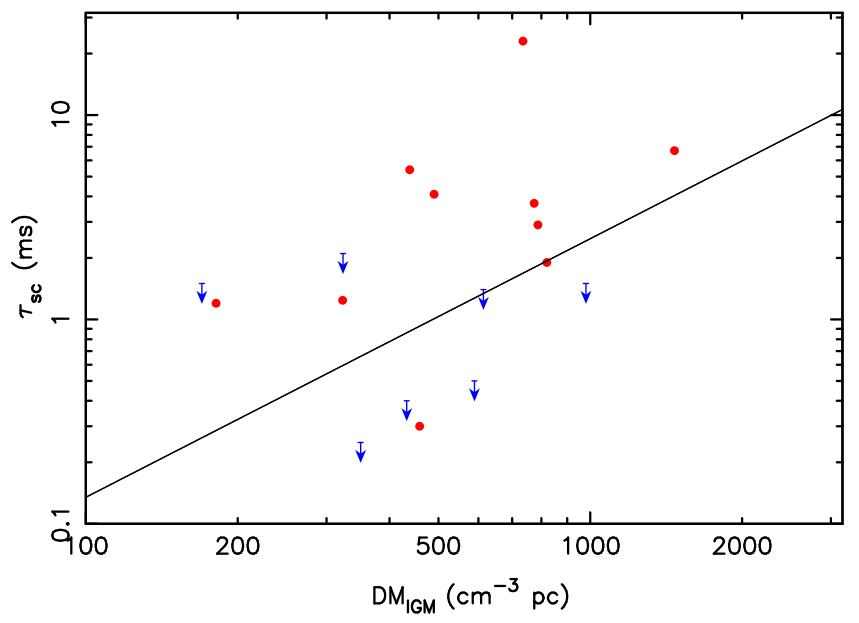

Figure 2. Observed FRB scattering times, normalised to $1 \mathrm{GHz}$ assuming $\tau_{\mathrm{sc}} \sim \nu^{-4.0}$, versus $\mathrm{DM}_{\mathrm{IGM}}=\mathrm{DM}_{-}-\mathrm{DM}_{\mathrm{Gal}}-\mathrm{DM}_{\mathrm{MC}}-$ $\mathrm{DM}_{\text {Host }}$, where we have assumed $\mathrm{DM}_{\text {Host }}=100 \mathrm{~cm}^{-3} \mathrm{pc}$. The fitted line is derived using a "survival analysis" technique to properly account for the upper limits on $\tau_{\mathrm{sc}}$.

Observationally, as Figure 2 shows, there is a clear tendency for scattering times to increase with DM $\mathrm{IGM}_{\mathrm{I}}$, supporting the idea that the IGM is a significant, if not the major contributor to the observed DM. In view of the relatively large number of upper limits, we have used a "survival analysis" technique, implemented in the routine ASURV (Lavalley et al. 1992), to fit a straight line to this trend, giving the result

$$
\log \left(\tau_{\mathrm{IGM}}\right)=(1.27 \pm 0.72) \log \left(\mathrm{DM}_{\mathrm{IGM}}\right)-(3.4 \pm 2.0)
$$

with a $90 \%$ probability that the correlation is real. Compared to the Krishnakumar et al. (2015) relation for Galactic 
scattering which is basically proportional to $\mathrm{DM}^{2.2}$, FRB scattering evidently has a somewhat flatter DM-dependence, $\sim \mathrm{DM}^{1.3}$, although the index has large uncertainty. The observed $\tau_{\mathrm{sc}}$ values implicitly include the lever-arm effect, and given the small number and large scatter of the observed $\tau_{\mathrm{sc}}$ values, we do not attempt to take the redshift dependencies (Macquart \& Koay 2013) into account at this stage.

Therefore, following the same reasoning as that used for the Magellanic Clouds, we take

$$
\tau_{\mathrm{FRB}}=\max \left(\tau_{\mathrm{Gal}}, \tau_{\mathrm{MC}}, \tau_{\mathrm{IGM}}, \tau_{\mathrm{Host}}\right) .
$$

as a predictor of FRB scattering times. Finally, we note that the ability to vary the assumed $D M_{\text {Host }}$, goes some way toward accommodating possible different assumptions about the origin of FRB scattering.

\section{DATA FITTING}

We wish to fit the electron density model described in the previous section to the data set comprising $N_{D}=189$ Galactic pulsars with independently determined distances and the 27 Magellanic Cloud pulsars with known DMs as described in $\S 2$ and listed in Tables A1 - A6. Seeking a global solution to this problem requires an algorithm that is optimised for non-linear, derivative-free and multi-dimensional parameter optimisation. We have investigated three such algorithms. The first is the modified evolutionary algorithm ESCH from the NLOPT library of non-linear optimisation routines (da Silva Santos et al. 2010) ${ }^{8}$ which analyses the generational evolution of candidate solutions. The second, PSwarm (Vaz \& Vincente 2007) ${ }^{9}$, is based on a "particle swarm" algorithm and the third is PoLYChord (Handley et al. 2015) ${ }^{10}$, a Bayesian routine using Markov-Chain Monte Carlo nested sampling methods. Both PSwarm and PolyChord are enabled for use with the Message Passing Interface MPI ${ }^{11}$ enabling faster execution on multi-processor computing systems. In each case, we have followed the global optimisation with a local parameter optimisation using the Nelder-Mead simplex algorithm MEAD routine from the NLOPT library.

As a goodness-of-fit statistic we seek to minimise the following function:

$$
F=\sum_{n=1}^{N_{D}} F_{n}
$$

where $F$ is summed over all (Galactic) pulsars with DM-independent distances and

$$
F_{n}= \begin{cases}\ln \left(\frac{D_{l}}{D_{m}}\right), & D_{m}<D_{l} \\ 0.5\left[\left(\frac{D_{i}-D_{m}}{D_{i}-D_{l}}\right)^{2}-1\right], & D_{l} \leq D_{m}<D_{i} \\ 0.5\left[\left(\frac{D_{m}-D_{i}}{D_{u}-D_{i}}\right)^{2}-1\right], & D_{i} \leq D_{m} \leq D_{u} \\ \ln \left(\frac{D_{m}}{D_{u}}\right), & D_{m}>D_{u}\end{cases}
$$

with $D_{m}$ is the model distance for a given pulsar, $D_{i}$ is the best estimate of its DM-independent distance and $D_{l}, D_{u}$ are the lower and upper limits to the independent distance, respectively. Figure 3 shows the goodness-of-fit function for PSR J1048-5832, chosen to illustrate the function for a pulsar with asymmetric and widely spaced limits.

Although all three optimisation routines we tested gave consistent global solutions, we found that PSwARM + MEAD gave the best results in terms of the final value of the goodness-of-fit statistic $F$ and speed of execution, and so the results presented here are based on these routines.

As described in $\S 3$, we fix many parameters of the model based on independent information, e.g., the form and location of the spiral arms, the size and shape of the Galactic Center disk, and the central position, distance and form of features such as the Gum Nebula and the Magellanic Clouds. There are no free parameters in the model for the intergalactic medium (see §3.10). Even after fixing these parameters, the number of model parameters to be fitted for is a significant fraction of the number of data values, which is a recipe for large covariances between parameters and poor convergence of the fitting process. For some parameters that are poorly constrained because of the small number of pulsars whose line-of-sight path passes through the particular model component, we fix additional parameters based on the results of preliminary fits to the data set.

\footnotetext{
8 http://ab-initio.mit.edu/nlopt

9 http://www.norg. uminho.pt/aivaz/pswarm

10 https://ccpforge.cse.rl.ac.uk/gf/project/polychord

${ }^{11}$ For a description of MPI, see, e.g., https://software.intel.com/en-us/articles/mpi-parallelizes-work-among-multiple-processors-or-hosts
} 


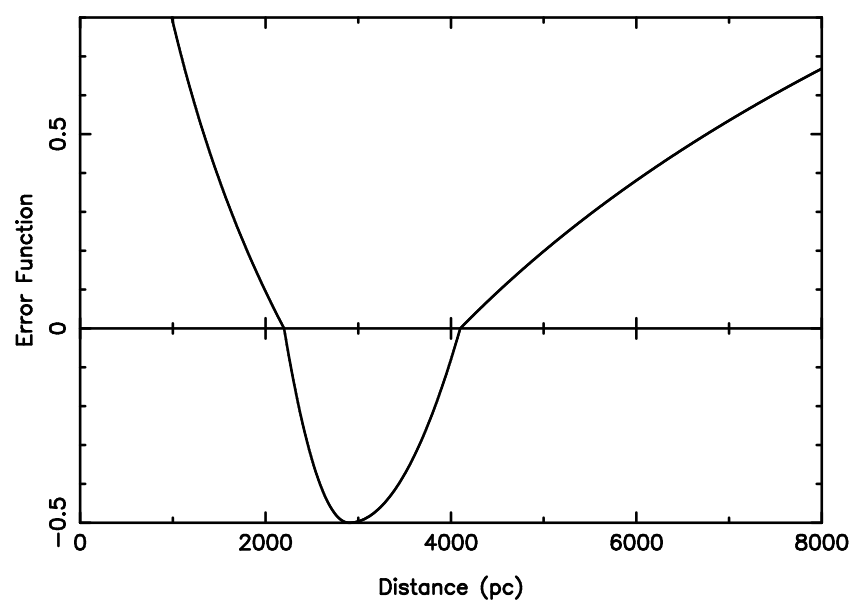

Figure 3. Error function $F$ for PSR J1048-5832. For this pulsar the DM-independent distance estimate is $D_{i}=2900$ pc, with lower and upper limits of $D_{l}=2200 \mathrm{pc}$ and $D_{u}=4100 \mathrm{pc}$, respectively (Verbiest et al. 2012).

The Fermi Bubbles are a special case. As described in §3.1, we modeled these as large cavities in the thick disk above and below the Galactic Center with electron densities given by $J_{\mathrm{FB}} n_{1}$, where $n_{1}$ is the local thick-disk electron density. Only two pulsars (PSRs J1748-2021A and J1750-3703A, associated with globular clusters NGC 6440 and NGC 6441 at distances $8.2 \pm 0.6 \mathrm{kpc}$ and $13.5 \pm 1.0 \mathrm{kpc}$, respectively) are within or behind the Fermi Bubbles. Preliminary fits for $J_{\mathrm{FB}}$ had relatively large uncertanties, but were consistent with a value of 1.0. In view of the large uncertainties, we decided to fix $J_{\mathrm{FB}}$ at 1.0. This obviously means that the Fermi Bubbles currently have no effect. However, we did not remove them from the model, as future pulsar discoveries and distance determinations will undoubtedly be made, allowing a more realistic determination of $J_{\mathrm{FB}}$ in a future version of the model.

The three adjustable parameters of the model for the Magellanic Clouds are separately fitted using just the pulsars known or believed to lie in the Magellanic Clouds (Table A6) while holding the Galactic model fixed. As is discussed further in $\S 5.4$, J0131-7301 was omitted from the fit because of its relatively large DM compared to the DMs of the other SMC pulsars.

Overall, the YMW16 model has 117 parameters, of which 35 are fitted by the parameter optimisation and 82 are fixed. Of the fitted parameters, 32 are for Galactic components of the model and three are for the Magellanic Clouds. Of the fixed parameters, 64 define the location, size and shape of Galactic components, including 15 defining the location of the spiral arms (Table 1). In addition to the three fitted parameters, the Magellanic Cloud components are defined by 15 fixed parameters. The model for the IGM has three fixed parameters, one of which, $\mathrm{DM}_{\text {Host }}$, the contribution of the FRB host galaxy to the observed DM, is adjustable but not fitted for.

As a means of assessing the performance of the model (and earlier models) we define a "distance error", $D_{\text {err }}$ for each pulsar having an independent distance estimate as follows:

$$
D_{e r r}= \begin{cases}0, & D_{l} \leq D_{m} \leq D_{u} \\ \frac{D_{l}}{D_{m}}-1, & D_{m}<D_{l} \\ \frac{D_{m}}{D_{u}}-1, & D_{m}>D_{u}\end{cases}
$$

In general, if $D_{\text {err }}$ is less than 20 per cent, we consider the model distance satisfactory.

As a check of the convergence of the model fit, we repeated the fitting 100 times randomly choosing initial values for the parameters within their allowed range. There was no significant evidence for secondary error-function minima in any of the parameters, although a few of the more poorly determined ones had a relatively wide and non-Gaussian distribution. Except for these cases, the peak of the distribution was used as the starting value for each parameter in the final global fit. For the non-gaussian cases a central starting value was chosen.

Parameter uncertainties are not easy to estimate because of the relatively small number of data points compared to the number of fitted parameters. The "bootstrap with replacement" method ${ }^{12}$ gave a good indication of uncertainties for some parameters, but not in cases where the parameter was determined by only a few data points, e.g., the central density of Galactic Center disk, which is determined by just one pulsar, PSR J1745-2900. As an alternative, we chose to

12 See, e.g., Efron (1981) for a description of bootstrap methods. 
estimate parameter uncertainties by randomly varying the values of $D_{i}$ within the range $D_{l}$ to $D_{u}$ for all pulsars in the data set and then refitting for the model parameters. This was repeated 200 times to give a distribution of values for each model parameter. These distributions were generally approximately Gaussian and hence gave reasonable values for the 1- $\sigma$ parameter uncertainties. The principal exceptions were $n_{a_{1}}$, the electron density of the Norma-Outer arm, and $n_{\mathrm{GC}_{0}}$, the central density of Galactic Center disk. As discussed above, $n_{\mathrm{GC}_{0}}$ was determined by just one pulsar and so this procedure would not be expected to lead to reliable results. For $n_{a_{1}}$, wide scatter may result from the fact that, within the zone of the thin disk, the larger of $n_{a_{i}}$ and $n_{2}$, the density of the thin disk, was taken as the model density (see §3.3). Despite these issues, we took the rms scatter of the 200 trial values to represent the 1- $\sigma$ uncertainties for all fitted parameters.

\section{RESULTS}

\subsection{The Galaxy}

Table 2 gives the allowed ranges, final-fit values and uncertainties for the 35 fitted parameters of the model and the assumed values for the 19 of the fixed parameters that are based on preliminary fits to the data set or independent evidence about the structure of model components. Other fixed parameters are described in $\S 3$.

Table 2. Parameters for the YMW16 electron-density model

\begin{tabular}{|c|c|c|c|c|c|}
\hline Component & Parameter & Lower limit & Upper limit & Value & Uncertainty \\
\hline \multirow[t]{4}{*}{ Thick disk } & $A_{d}(\mathrm{pc})$ & 2500 & 2500 & 2500 & - \\
\hline & $B_{d}(\mathrm{pc})$ & 15000 & 15000 & 15000 & - \\
\hline & $n_{1_{0}}\left(\mathrm{~cm}^{-3}\right)$ & 0.008 & 0.016 & 0.01132 & 0.00043 \\
\hline & $H_{1}(\mathrm{pc})$ & 1200 & 2000 & 1673 & 53 \\
\hline \multirow[t]{4}{*}{ Thin disk } & $A_{2}(\mathrm{pc})$ & 1200 & 1200 & 1200 & - \\
\hline & $B_{2}(\mathrm{pc})$ & 4000 & 4000 & 4000 & - \\
\hline & $n_{2_{0}}\left(\mathrm{~cm}^{-3}\right)$ & 0.3 & 0.5 & 0.404 & 0.051 \\
\hline & $K_{2}$ & 1.0 & 3.0 & 1.54 & 0.16 \\
\hline \multirow[t]{18}{*}{ Spiral arms } & $n_{a_{1}}\left(\mathrm{~cm}^{-3}\right)$ & 0.03 & 0.15 & 0.135 & 0.024 \\
\hline & $n_{a_{2}}\left(\mathrm{~cm}^{-3}\right)$ & 0.03 & 0.15 & 0.129 & 0.011 \\
\hline & $n_{a_{3}}\left(\mathrm{~cm}^{-3}\right)$ & 0.03 & 0.15 & 0.103 & 0.006 \\
\hline & $n_{a_{4}}\left(\mathrm{~cm}^{-3}\right)$ & 0.03 & 0.15 & 0.116 & 0.008 \\
\hline & $n_{a_{5}}\left(\mathrm{~cm}^{-3}\right)$ & 0.003 & 0.02 & 0.0057 & 0.0013 \\
\hline & $w_{a_{1}}(\mathrm{pc})$ & 300 & 300 & 300 & - \\
\hline & $w_{a_{2}}(\mathrm{pc})$ & 500 & 500 & 500 & - \\
\hline & $w_{a_{3}}(\mathrm{pc})$ & 300 & 300 & 300 & - \\
\hline & $w_{a_{4}}(\mathrm{pc})$ & 500 & 500 & 500 & - \\
\hline & $w_{a_{5}}(\mathrm{pc})$ & 300 & 300 & 300 & - \\
\hline & $A_{a}(\mathrm{pc})$ & 7000 & 15000 & 11680 & 1490 \\
\hline & $K_{a}$ & 3.0 & 6.0 & 5.01 & 0.15 \\
\hline & $n_{\mathrm{CN}}$ & 1.0 & 3.0 & 2.40 & 0.26 \\
\hline & $\phi_{\mathrm{CN}}\left(^{\circ}\right)$ & 109 & 109 & 109 & - \\
\hline & $\Delta \phi_{\mathrm{CN}}$ & 2.0 & 15.0 & 8.2 & 1.1 \\
\hline & $n_{\mathrm{SG}}$ & 0.1 & 1.0 & 0.626 & 0.068 \\
\hline & $\phi_{\mathrm{SG}}\left({ }^{\circ}\right)$ & 60 & 100 & 75.8 & 2.2 \\
\hline & $\Delta \phi_{\mathrm{SG}}\left(^{\circ}\right)$ & 20 & 20 & 20 & - \\
\hline Galactic warp & $\gamma_{w}$ & 0 & 0.3 & 0.140 & 0.066 \\
\hline \multirow[t]{3}{*}{ Galactic Ctr } & $n_{\mathrm{GC}_{0}}\left(\mathrm{~cm}^{-3}\right)$ & 1.0 & 10.0 & 6.2 & 2.6 \\
\hline & $A_{\mathrm{GC}}(\mathrm{pc})$ & 160 & 160 & 160 & - \\
\hline & $H_{\mathrm{GC}}(\mathrm{pc})$ & 35 & 35 & 35 & - \\
\hline \multirow[t]{3}{*}{ Gum Nebula } & $n_{\mathrm{GN}_{0}}\left(\mathrm{~cm}^{-3}\right)$ & 1.0 & 3.0 & 1.84 & 0.12 \\
\hline & $W_{\mathrm{GN}}(\mathrm{pc})$ & 10 & 20 & 15.1 & 0.8 \\
\hline & $A_{\mathrm{GN}}(\mathrm{pc})$ & 120 & 130 & 125.8 & 0.8 \\
\hline
\end{tabular}


Table 2 (continued)

\begin{tabular}{|c|c|c|c|c|c|}
\hline Component & Parameter & Lower limit & Upper limit & Value & Uncertainty \\
\hline \multirow{12}{*}{ Local Bubble } & $K_{\mathrm{GN}}$ & 1.4 & 1.4 & 1.4 & - \\
\hline & $J_{\mathrm{LB}}$ & 0.4 & 1.2 & 0.480 & 0.063 \\
\hline & $n_{\mathrm{LB} 1_{0}}\left(\mathrm{~cm}^{-3}\right)$ & 0.5 & 1.5 & 1.094 & 0.073 \\
\hline & $\theta_{\mathrm{LB} 1}\left(^{\circ}\right)$ & 190 & 200 & 195.4 & 1.2 \\
\hline & $\Delta \theta_{\mathrm{LB} 1}\left(^{\circ}\right)$ & 20 & 40 & 28.4 & 1.1 \\
\hline & $W_{\mathrm{LB} 1}(\mathrm{pc})$ & 10 & 20 & 14.2 & 0.9 \\
\hline & $H_{\mathrm{LB} 1}(\mathrm{pc})$ & 80 & 130 & 112.9 & 3.9 \\
\hline & $n_{\mathrm{LB} 2_{0}}\left(\mathrm{~cm}^{-3}\right)$ & 1.0 & 3.0 & 2.33 & 0.15 \\
\hline & $\theta_{\mathrm{LB} 2}\left(^{\circ}\right)$ & 260 & 300 & 278.2 & 1.1 \\
\hline & $\Delta \theta_{\mathrm{LB} 2}\left(^{\circ}\right)$ & 10 & 60 & 14.7 & 0.7 \\
\hline & $W_{\mathrm{LB} 2}(\mathrm{pc})$ & 10 & 20 & 15.6 & 1.1 \\
\hline & $H_{\mathrm{LB} 2}(\mathrm{pc})$ & 10 & 60 & 43.6 & 2.6 \\
\hline \multirow[t]{5}{*}{ Loop I } & $n_{\mathrm{LI}_{0}}\left(\mathrm{~cm}^{-3}\right)$ & 0.0 & 3.0 & 1.907 & 0.096 \\
\hline & $R_{\mathrm{LI}}(p c)$ & 80 & 80 & 80 & - \\
\hline & $W_{\mathrm{LI}}(p c)$ & 15 & 15 & 15 & - \\
\hline & $\Delta \theta_{\mathrm{LI}}\left(^{\circ}\right)$ & 30 & 30 & 30 & - \\
\hline & $\theta_{\text {LI }}\left(^{\circ}\right)$ & 40 & 40 & 40 & - \\
\hline Fermi Bubbles & $J_{\mathrm{FB}}$ & 1.0 & 1.0 & 1.0 & - \\
\hline \multirow[t]{2}{*}{ LMC } & $n_{\mathrm{LMC}_{0}}\left(\mathrm{~cm}^{-3}\right)$ & 0.05 & 0.3 & 0.066 & 0.007 \\
\hline & $n_{30 \mathrm{D}_{0}}\left(\mathrm{~cm}^{-3}\right)$ & 0.05 & 0.5 & 0.32 & 0.17 \\
\hline $\mathrm{SMC}$ & $n_{\mathrm{SMC}_{0}}\left(\mathrm{~cm}^{-3}\right)$ & 0 & 0.3 & 0.045 & 0.017 \\
\hline
\end{tabular}

The model electron density in the Galactic plane $(z=0)$ is illustrated in Figure 4. This figure emphasizes the high degree of symmetry of our model. As discussed in $\S 1$, we have tried to minimise the number of special features, only defining one when a localised group of pulsars showed consistently over-estimated or under-estimated model distances. Figure 4 also illustrates the way in which the spiral arms emerge from the thin disk annulus.

The radial dependence of the electron density on the Galactic plane from the Galactic Center outwards in the direction of the Sun is shown in Figure 5. In the inner Galaxy the Galactic Center disk and the thin disk dominate, whereas in the outer Galaxy, the spiral arms dominate. At higher Galactic latitudes the thick disk is dominant. We have also plotted the total model Galactic DM at $b=0$ as a function of Galactic longitude $l$ in Figure 1 . This clearly shows the asymmetry in the spiral structure with much greater integrated electron densities in the fourth Galactic quadrant, especially for the Carina arm. Since most known pulsars are relatively local and at non-zero Galactic latitude, most points in Figure 1 are well below the maximum DM line. Exceptions are PSR J0248+6021 (at $\left.l=137^{\circ}\right)$, believed to lie close to the HII region W5 (§2.4), and four pulsars near $l=305^{\circ}$ in the Carina region. Our model already has an increased electron density in the Carina arm (§3.3) but these four pulsars clearly have a greater-than-average contribution from HII regions in the path.

For the thick disk, the YMW16 model gives $n_{1_{0}}=0.01132 \pm 0.00043 \mathrm{~cm}^{-3}$ and $H_{1}=1673 \pm 53 \mathrm{pc}$ (Table 2), corresponding to a $n_{1_{0}} H_{1}$ product of $18.9 \pm 0.9 \mathrm{~cm}^{-3}$ pc. This is just over half of the perpendicular DM for the NE2001 model, but comparable to those for the TC93, Gaensler et al. (2008) and Savage \& Wakker (2009) models. Correspondingly, the YMW16 mid-plane density is about one third the NE2001 value and the scale height is about $70 \%$ larger than the NE2001 value. However, the YMW16 scale-height estimate is consistent within the uncertainties with the estimates of Gaensler et al. (2008), Savage \& Wakker (2009) and Schnitzeler (2012).

As Figures 4 and 5 show, the thin disk is relatively dense with a peak $n_{e} \sim 0.4 \mathrm{~cm}^{-3}$ at $R=4 \mathrm{kpc}$. Although the thin disk doesn't extend very far in Galactocentric radius, based on the independent measurements discussed in $\S 3.2$, we use Equation 8 to define the scale height dependence on $R$ and fit for the scale factor $K_{2}$. This scale height dependence is illustrated in Figure 6.

Except for the Local arm, the reference spiral arm electron densities $n_{a_{i}}$ at $R=4 \mathrm{kpc}$ are all about $0.1 \mathrm{~cm}^{-3}$ (Table 2). The Local arm is much less dense, with $n_{a_{5}}$ just $0.0057 \mathrm{~cm}^{-3}$, only about half of the density of the thick disk. The radial scale length of the spiral arms $A_{a}$, common to all arms, is relatively large, $11.7 \pm 1.5 \mathrm{kpc}$, and the 


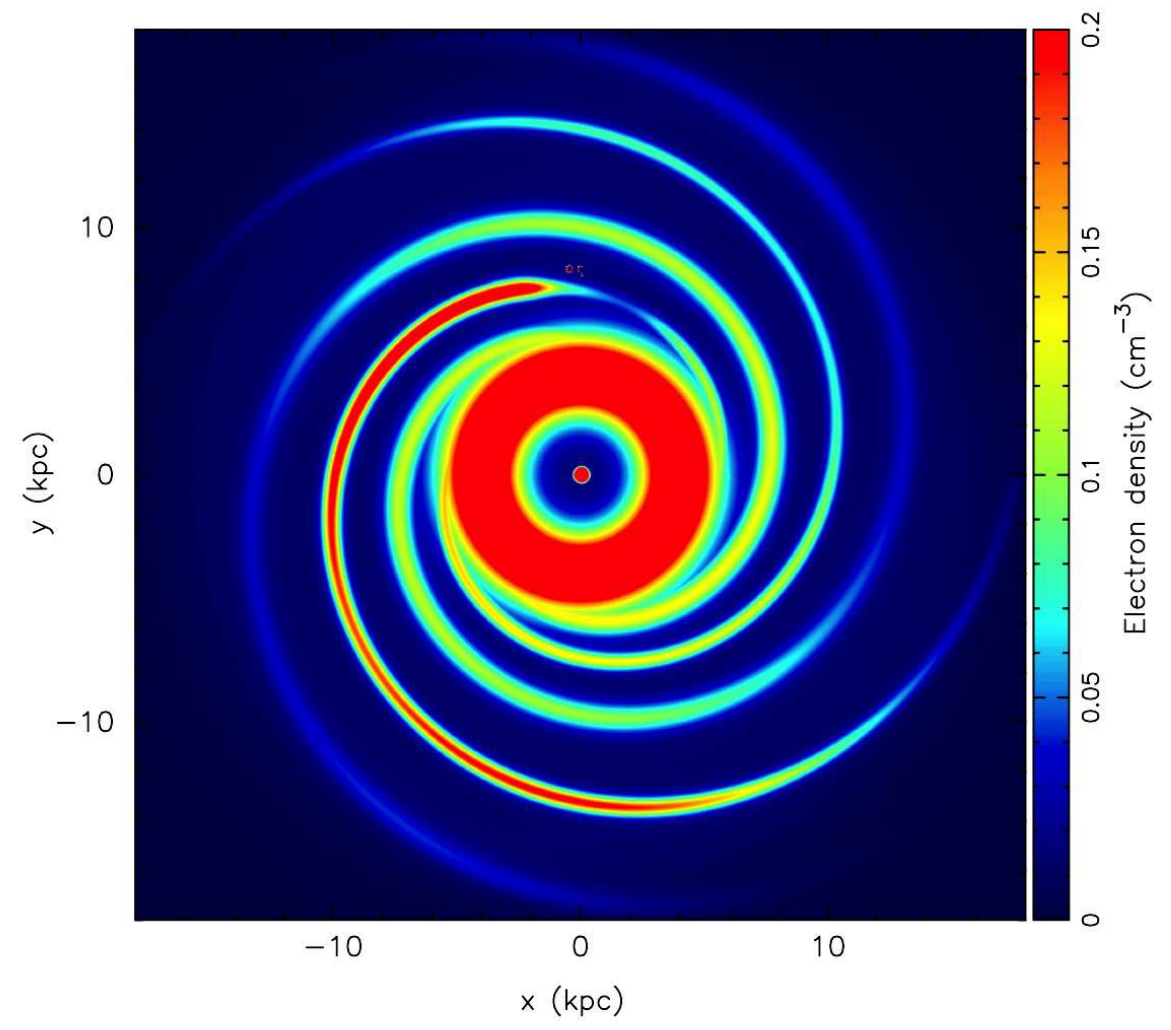

Figure 4. Electron density in the Galactic plane $(z=0)$ for the YMW16 model. The Galactic Center is at the origin and the Sun is at $x=0, y=+8.5 \mathrm{kpc}$. The dense annulus of central radius $4 \mathrm{kpc}$ is the "thin disk" which represents the Galactic molecular ring. Spiral arms have a pre-determined logarithmic spiral form and generally decay exponentially at large Galactocentric radii. Exceptions are in the Carina - Sagittarius arm where there are over-dense and under-dense regions in Carina and Sagittarius respectively. The Gum Nebula and Local Bubble features are faintly visible near the position of the Sun.

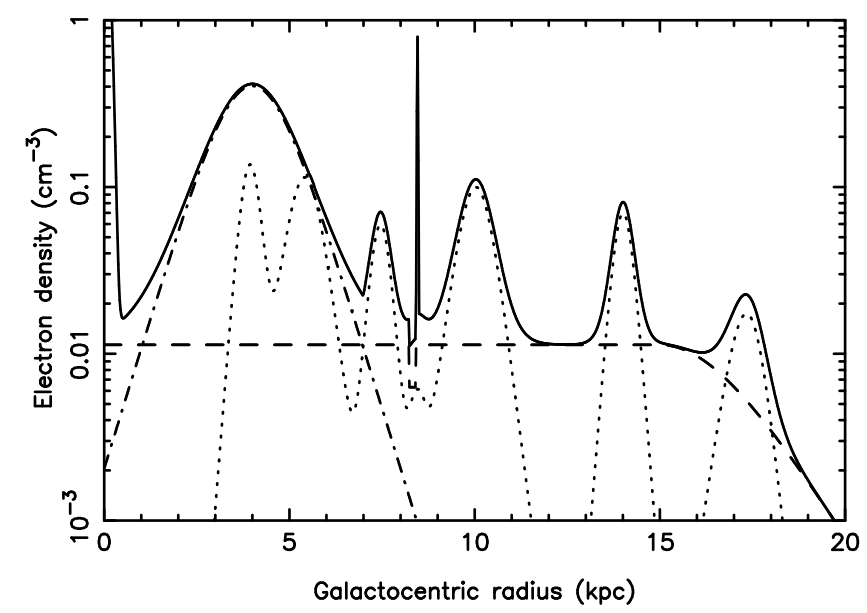

Figure 5. Electron density versus Galactocentric radius along the $+y$ axis (toward the Sun) for the YMW16 model. The major components are shown separately: thick disk (dashed), thin disk (dot-dashed) and spiral arms (dotted). The Galactic Center component peaks at $5.8 \mathrm{~cm}^{-3} \mathrm{pc}$ and the Local Bubble depression and associated over-density LB1 can be seen at $8.5 \mathrm{kpc}$.

scale-height factor $K_{a}$ is $5.01 \pm 0.15$ (Figure 5). The over-dense Carina region and under-dense Sagittarius region 




Figure 6. Dependence of thin-disk and spiral-arm scale height on Galactocentric radius. The full line corresponds to the function $H$ (Equation 8), the dot-dashed line to the thin disk with $K_{2} \approx 1.5$ and the dashed line is for the spiral arms with $K_{a} \approx 5.0$.

reflect the asymmetry seen in the latitude distribution of DMs (Figure 1). Parameters of these regions, defined by Equation 12, are given in Table 2. Angular widths were estimated from preliminary fits and were held fixed for the global fit.

The Galactic warp only affects only a few pulsars with our present data set. We never-the-less have fitted for its amplitude and obtain a value for $\gamma_{w}=0.140 \pm 0.066$. This is a little less than, but consistent with, the value of 0.18 adopted by Robin et al. (2003). The largest change resulting from introduction of the warp is for PSR J2229+6114, where the model distance is reduced by $\sim 870 \mathrm{pc}$ to $\sim 5040 \mathrm{pc}$, moving it closer to the independent distance of $3000 \mathrm{pc}$ (Table A4). Eleven pulsars with independent distances have their model distance changed by $100 \mathrm{pc}$ or more.

The Galactic Center disk and the Gum Nebula ellipsoid are relatively straight-forward. As mentioned in $\S 4$, the Galactic Center electron density is currently determined by just one pulsar, but eight pulsars (including the Vela pulsar and the Double Pulsar) lie within or beyond the Gum Nebula and have a DM contribution from it. The derived radius of the ellipsoidal shell in the $x-y$ plane is about $125 \mathrm{pc}$, the shell half-thickness is $W_{\mathrm{GN}} \sim 15 \mathrm{pc}$ and the electron density at the shell mid-line is $n_{\mathrm{GN}_{0}} \sim 1.8 \mathrm{~cm}^{-3}$. Consequently, the DM contribution of a perpendicular traverse of one side of the shell is $\sqrt{\pi} n_{\mathrm{GN}_{0}} W_{\mathrm{GN}} \sim 50 \mathrm{~cm}^{-3} \mathrm{pc}$.

As discussed in $§ 3.6$, there is good evidence for a relatively low-density region surrounding the Sun. The size and shape of this region in our model is determined by the stellar absorption results and we just fit for the internal electron density as a multiple of the thick-disk density as part of the global fit. The derived scale factor $J_{\mathrm{LB}} \sim 0.48$ indicating that, on average, $n_{e}$ within the Local Bubble is about half the density of the surrounding thick disk plus local spiral arm contributions. The stellar absorption results also indicate the presence of relatively dense swept-up regions surrounding the Local Bubble. We modelled these as described in $\S 3.6$, obtaining the parameters listed in Table 2 as part of the global fit. The two modelled over-dense regions cover about half of the circumference of the local bubble with central electron densities of $1.0 \mathrm{~cm}^{-3}$ and $2.3 \mathrm{~cm}^{-3}$ respectively.

Most of the parameters of Loop I (and the roughly hemispherical ionised shell which appears to be associated with the North Polar Spur) were held fixed as discussed in §3.7. Only the reference electron density, defined by Equation 21, was solved for as part of the global fit; the derived value is quite high, about $1.9 \mathrm{~cm}^{-3}$, suggesting the presence of a relatively dense ionised shell associated with the North Polar Spur.

Four representative plots showing the contribution of most model components to the DM as a function of distance along the path to the pulsar are shown in Figure 7. The top-left plot is for PSR J1745-2900 which is located very close to the Galactic Center. The dominant contributors to the DM are the thin disk, spiral arms and, near the pulsar, the Galactic Center disk. This pulsar is the only one determining the density of the Galactic Center disk which is adjusted by the fit to correctly model the pulsar distance. The top-right plot is for a pulsar behind the Gum Nebula with the plot showing the increments of about $50 \mathrm{~cm}^{-3} \mathrm{pc}$ with the traversal of the near side and far side of the ellipsoidal shell. At $\sim 100 \mathrm{pc}$ distance, the Local Bubble feature LB2 contributes about $30 \mathrm{~cm}^{-3} \mathrm{pc}$.

A pulsar, PSR J0248+6021, whose distance is greatly over-estimated is shown in the bottom-left plot. Only the thick disk and spiral arms contribute to the DM of this pulsar and they are insufficient to account for the DM of $370 \mathrm{~cm}^{-3} \mathrm{pc}$. In the discovery paper for this pulsar, Theureau et al. (2011) argue that this pulsar is associated with the giant HII region W5 at a distance of approximately $2 \mathrm{kpc}$ and this distance is adopted as the independent distance. The excess 
DM (i.e., DM - $\mathrm{DM}_{\mathrm{Gal}}$ ), about $160 \mathrm{~cm}^{-3} \mathrm{pc}$ with the NE2001 model and about $60 \mathrm{~cm}^{-3} \mathrm{pc}$ with our model can be attributed to W5. In contrast, the bottom-right plot shows the DM components for PSR J1744-1134, a relatively nearby millisecond pulsar whose distance is significantly under-estimated by the model. This plot shows that the underestimate is largely due to a contribution from Loop I, building up from about $120 \mathrm{pc}$ distance. This contribution is clearly not needed for this pulsar. However, of the 13 pulsars within or behind Loop I, ten have satisfactory model distances $\left(D_{\text {err }}<20 \%\right)$, one is over-estimated and two (including PSR J1744-1134) are under-estimated. This shows that, on average, Loop I makes a useful contibution to the model but that, not surprisingly, reality is more complex than our relatively simple hemispherical shell model. The PSR J1744-1134 plot is also interesting in that it shows the effect of the Local Bubble on the contribution from the thick disk, with the DM gradient increasing sharply at approximately $70 \mathrm{pc}$, the edge of the Local Bubble in this direction.


Figure 7. Plots showing the contributions from different components of the model to the pulsar DM as a function of distance along the path for four selected pulsars. For each subplot, the pulsar name, the Galactic coordinates, the DM, the independent distance estimate and the model distance estimate are given. The upper two plots are for pulsars with $D_{\text {err }} \leq 20 \%$, the lower-left plot is for a pulsar with an over-estimated distance and the lower-right plot is for a pulsar with an under-estimated distance. Model components are as follows: thick disk (red dotted line), thin disk (blue dot-dashed line), spiral arms (cyan dashed line), Local Bubble shell (magenta line), Gum Nebula (orange line), Loop I (green line), Galactic Center disk (orange dashed line) and the total (full black line). The red circle on each subplot marks the independent distance $D_{i}$ and the observed DM. For PSR J1744-1134, the actual $D_{i}$ is beyond the right edge of the subplot.

Model distances and associated $D_{\text {err }}$ values are listed for all 189 Galactic pulsars with independent distances in Appendix TablesA1 - A5. Of these 189 Galactic pulsars, 86 have model distances within the uncertainties of the independent distances and hence have $D_{e r r}=0$ and a further 38 have $D_{e r r} \leq 20 \%$, giving 124 independent distances, or $65 \%$ of the total, that are satisfactorily represented by the model. Of the 65 pulsars with $D_{\text {err }}>20 \%, 35$ have over-estimated distances, that is insufficient $n_{e}$ along the path to account for the DM, and 30 have under-estimated distances. These pulsars are listed in Tables 3 and 4 respectively. Figures 8 and 9 show the distribution of the 189 pulsars in Galactic coordinates and projected onto the Galactic plane, respectively, with over-estimated and under- 
estimated distances marked by different symbols. Within statistical fluctuations, under-estimated, over-estimated and correctly estimated distances are reasonably evenly distributed in both projections.

Table 3. Parameters for 35 pulsars with overestimated distances

\begin{tabular}{|c|c|c|c|c|c|c|c|c|}
\hline $\begin{array}{l}\text { J2000 } \\
\text { Name }\end{array}$ & $\begin{array}{c}l \\
\left({ }^{\circ}\right)\end{array}$ & $\begin{array}{c}b \\
\left({ }^{\circ}\right)\end{array}$ & $\begin{array}{c}\mathrm{DM} \\
\left(\mathrm{cm}^{-3} \mathrm{pc}\right)\end{array}$ & $\begin{array}{c}D_{1} \\
(\mathrm{pc})\end{array}$ & $\begin{array}{c}D_{i} \\
(\mathrm{pc})\end{array}$ & $\begin{array}{l}D_{u} \\
(\mathrm{pc})\end{array}$ & $\begin{array}{l}D_{m} \\
(\mathrm{pc})\end{array}$ & $\begin{array}{c}D_{e r r} \\
(\%)\end{array}$ \\
\hline $\mathrm{J} 2144-3933$ & 2.794 & -49.466 & 3.35 & 150 & 160 & 180 & 289 & 61 \\
\hline $\mathrm{J} 1756-2251$ & 6.499 & 0.948 & 121.18 & 490 & 730 & 1330 & 2806 & 111 \\
\hline $\mathrm{J} 1801-2304$ & 6.837 & -0.066 & 1073.9 & 3000 & 4000 & 5000 & 6522 & 30 \\
\hline $\mathrm{J} 1824-1945$ & 12.279 & -3.106 & 224.65 & 2800 & 3700 & 4300 & 5612 & 30 \\
\hline $\mathrm{J} 1543+0929$ & 17.811 & 45.775 & 35.24 & 5400 & 5900 & 6500 & 25000 & 285 \\
\hline $\mathrm{J} 1820-0427$ & 25.456 & 4.733 & 84.44 & 100 & 300 & 900 & 2918 & 224 \\
\hline $\mathrm{J} 1903+0135$ & 35.727 & -1.955 & 245.17 & 2800 & 3300 & 3900 & 6000 & 54 \\
\hline $\mathrm{J} 1901+0716$ & 40.569 & 1.056 & 252.81 & 2700 & 3400 & 4300 & 7237 & 68 \\
\hline $\mathrm{J} 1342+2822 \mathrm{~A}$ & 42.209 & 78.709 & 26.5 & 9600 & 9900 & 10200 & 25000 & 145 \\
\hline $\mathrm{J} 1939+2134$ & 57.509 & -0.29 & 71.04 & 1200 & 1500 & 2000 & 2897 & 45 \\
\hline $\mathrm{J} 2129+1210 \mathrm{~A}$ & 65.012 & -27.312 & 67 & 12900 & 13550 & 14200 & 25000 & 76 \\
\hline $\mathrm{J} 2021+3651$ & 75.222 & 0.111 & 367.5 & 400 & 1800 & 3500 & 10512 & 200 \\
\hline $\mathrm{J} 2032+4127$ & 80.224 & 1.028 & 114.65 & 1400 & 1500 & 1700 & 4623 & 172 \\
\hline $\mathrm{J} 2214+3000$ & 86.855 & -21.665 & 22.56 & 909 & 1000 & 1111 & 1674 & 51 \\
\hline $\mathrm{J} 2157+4017$ & 90.488 & -11.341 & 70.86 & 2500 & 2900 & 3400 & 4750 & 40 \\
\hline $\mathrm{J} 2229+6114$ & 106.647 & 2.949 & 204.97 & 2400 & 3000 & 3600 & 5037 & 40 \\
\hline $\mathrm{J} 2337+6151$ & 114.284 & 0.233 & 58.41 & 600 & 700 & 800 & 2079 & 160 \\
\hline $\mathrm{J} 0248+6021$ & 136.903 & 0.697 & 370 & 1800 & 2000 & 2200 & 25000 & 1036 \\
\hline $\mathrm{J} 0358+5413$ & 148.19 & 0.811 & 57.14 & 900 & 1000 & 1200 & 1594 & 33 \\
\hline J0452-1759 & 217.078 & -34.087 & 39.9 & 300 & 400 & 600 & 2710 & 352 \\
\hline $\mathrm{J} 0922+0638$ & 225.42 & 36.392 & 27.27 & 1000 & 1100 & 1300 & 1908 & 47 \\
\hline J0630-2834 & 236.952 & -16.758 & 34.47 & 280 & 320 & 370 & 2072 & 460 \\
\hline J0614-3329 & 240.501 & -21.827 & 37.05 & 760 & 890 & 1020 & 2691 & 164 \\
\hline J0514-4002A & 244.514 & -35.036 & 52.15 & 12100 & 12650 & 13200 & 25000 & 89 \\
\hline $\mathrm{J} 1017-7156$ & 291.558 & -12.553 & 94.22 & 196 & 256 & 370 & 1807 & 388 \\
\hline $\mathrm{J} 1243-6423$ & 302.051 & -1.532 & 297.25 & 0 & 2000 & 4000 & 9411 & 135 \\
\hline $\mathrm{J} 1326-5859$ & 307.504 & 3.565 & 287.3 & 2000 & 3000 & 5000 & 10688 & 113 \\
\hline $\mathrm{J} 1603-7202$ & 316.63 & -14.496 & 38.05 & 370 & 530 & 570 & 1129 & 98 \\
\hline $\mathrm{J} 1550-5418$ & 327.237 & -0.132 & 830 & 3500 & 4000 & 4500 & 6291 & 40 \\
\hline $\mathrm{J} 1312+1810$ & 332.954 & 79.763 & 24 & 17200 & 18900 & 20600 & 25000 & 21 \\
\hline $\mathrm{J} 2129-5721$ & 338.005 & -43.57 & 31.85 & 1700 & 3200 & 4700 & 6170 & 31 \\
\hline $\mathrm{J} 1623-2631$ & 350.976 & 15.96 & 62.86 & 1600 & 1800 & 2000 & 3651 & 82 \\
\hline $\mathrm{J} 1614-2230$ & 352.636 & 20.192 & 34.5 & 400 & 700 & 1000 & 1395 & 39 \\
\hline $\mathrm{J} 1740-3015$ & 358.294 & 0.238 & 152.15 & 100 & 400 & 2100 & 2945 & 40 \\
\hline $\mathrm{J} 1745-3040$ & 358.553 & -0.963 & 88.37 & 0 & 200 & 1300 & 2343 & 80 \\
\hline
\end{tabular}

Table 4. Parameters for 30 pulsars with underestimated distances

\begin{tabular}{|c|c|c|c|c|c|c|c|c|}
\hline $\begin{array}{l}\text { J2000 } \\
\text { Name }\end{array}$ & $\begin{array}{c}l \\
\left({ }^{\circ}\right)\end{array}$ & $\begin{array}{c}b \\
\left({ }^{\circ}\right)\end{array}$ & $\begin{array}{c}\mathrm{DM} \\
\left(\mathrm{cm}^{-3} \mathrm{pc}\right)\end{array}$ & $\begin{array}{c}D_{1} \\
(\mathrm{pc})\end{array}$ & $\begin{array}{c}D_{i} \\
(\mathrm{pc})\end{array}$ & $\begin{array}{l}D_{u} \\
(\mathrm{pc})\end{array}$ & $\begin{array}{l}D_{m} \\
(\mathrm{pc})\end{array}$ & $\begin{array}{c}D_{\text {err }} \\
(\%)\end{array}$ \\
\hline $\mathrm{J} 1835-3259 \mathrm{~A}$ & 1.532 & -11.371 & 63.35 & 10200 & 10700 & 11200 & 2711 & 276 \\
\hline $\mathrm{J} 1823-3021 \mathrm{~A}$ & 2.788 & -7.913 & 86.88 & 7800 & 8400 & 9000 & 3145 & 148 \\
\hline J1721-1936 & 4.857 & 9.738 & 75.7 & 7800 & 8400 & 9000 & 3070 & 154 \\
\hline
\end{tabular}


Table 4 (continued)

\begin{tabular}{|c|c|c|c|c|c|c|c|c|}
\hline $\begin{array}{l}\text { J2000 } \\
\text { Name }\end{array}$ & $\begin{array}{c}l \\
\left({ }^{\circ}\right)\end{array}$ & $\begin{array}{c}b \\
\left({ }^{\circ}\right)\end{array}$ & $\begin{array}{c}\mathrm{DM} \\
\left(\mathrm{cm}^{-3} \mathrm{pc}\right)\end{array}$ & $\begin{array}{c}D_{1} \\
(\mathrm{pc})\end{array}$ & $\begin{array}{c}D_{i} \\
(\mathrm{pc})\end{array}$ & $\begin{array}{l}D_{u} \\
(\mathrm{pc})\end{array}$ & $\begin{array}{l}D_{m} \\
(\mathrm{pc})\end{array}$ & $\begin{array}{c}D_{\text {err }} \\
(\%)\end{array}$ \\
\hline $\mathrm{J} 1824-2452 \mathrm{~A}$ & 7.797 & -5.578 & 120.5 & 5200 & 5500 & 5800 & 3737 & 39 \\
\hline $\mathrm{J} 1744-1134$ & 14.794 & 9.18 & 3.14 & 384 & 395 & 406 & 148 & 159 \\
\hline $\mathrm{J} 2140-2310 \mathrm{~A}$ & 27.179 & -46.837 & 25.06 & 9200 & 9450 & 9700 & 3112 & 196 \\
\hline $\mathrm{J} 1713+0747$ & 28.751 & 25.223 & 15.99 & 1136 & 1176 & 1220 & 919 & 24 \\
\hline $\mathrm{J} 1905+0154 \mathrm{~A}$ & 36.208 & -2.201 & 193.69 & 13950 & 14450 & 14950 & 5509 & 153 \\
\hline $\mathrm{J} 1906+0746$ & 41.598 & 0.147 & 217.75 & 6000 & 7400 & 9900 & 4814 & 25 \\
\hline $\mathrm{J} 1917+1353$ & 48.26 & 0.624 & 94.54 & 4000 & 5000 & 6000 & 2940 & 36 \\
\hline $\mathrm{J} 1953+1846 \mathrm{~A}$ & 56.744 & -4.563 & 117 & 6000 & 6450 & 6900 & 4505 & 33 \\
\hline $\mathrm{J} 1932+2220$ & 57.356 & 1.554 & 219.2 & 10100 & 10900 & 12200 & 7999 & 26 \\
\hline J0454+5543 & 152.617 & 7.547 & 14.49 & 1130 & 1180 & 1250 & 631 & 79 \\
\hline $\mathrm{J} 0337+1715$ & 169.99 & -30.039 & 21.32 & 1220 & 1300 & 1380 & 817 & 49 \\
\hline J0633+1746 & 195.134 & 4.266 & 2.89 & 170 & 250 & 480 & 138 & 23 \\
\hline J0659+1414 & 201.108 & 8.258 & 13.98 & 250 & 280 & 310 & 159 & 57 \\
\hline J0953+0755 & 228.908 & 43.697 & 2.96 & 256 & 261 & 266 & 186 & 37 \\
\hline $\mathrm{J} 1023+0038$ & 243.49 & 45.782 & 14.32 & 1328 & 1367 & 1410 & 1057 & 26 \\
\hline J1024-0719 & 251.702 & 40.516 & 6.49 & 800 & 1100 & 1500 & 381 & 110 \\
\hline J1048-5832 & 287.425 & 0.577 & 129.1 & 2200 & 2900 & 4100 & 1793 & 23 \\
\hline $\mathrm{J} 1119-6127$ & 292.151 & -0.537 & 707.4 & 8000 & 8400 & 8800 & 6414 & 25 \\
\hline $\mathrm{J} 1227-4853$ & 298.965 & 13.796 & 43.42 & 1800 & 1900 & 2000 & 1244 & 45 \\
\hline $\mathrm{J} 1224-6407$ & 299.984 & -1.415 & 97.47 & 2000 & 4000 & 6000 & 1534 & 31 \\
\hline $\mathrm{J} 0024-7204 \mathrm{C}$ & 305.923 & -44.892 & 24.4 & 3650 & 4000 & 4350 & 2547 & 43 \\
\hline $\mathrm{J} 1453-6413$ & 315.733 & -4.427 & 71.07 & 2000 & 2800 & 4100 & 1432 & 40 \\
\hline $\mathrm{J} 1602-5100$ & 330.688 & 1.286 & 170.93 & 7300 & 8000 & 8900 & 3407 & 114 \\
\hline J1559-4438 & 334.54 & 6.367 & 56.1 & 2000 & 2300 & 2800 & 1480 & 36 \\
\hline J1910-5959A & 336.525 & -25.73 & 33.28 & 4490 & 4550 & 4610 & 1642 & 174 \\
\hline $\mathrm{J} 1701-3006 \mathrm{~A}$ & 353.578 & 7.322 & 114.97 & 6470 & 7050 & 7630 & 4881 & 33 \\
\hline J1909-3744 & 359.731 & -19.596 & 10.39 & 1230 & 1234 & 1239 & 564 & 118 \\
\hline
\end{tabular}

Figure 10 shows an expanded view of Figure 9 for the local region. The Gum Nebula and Local Bubble are circular in the $x-y$ plane and, as Figure 11 illustrates, only the tip of the Loop I shell crosses the $x-y$ plane. With a peak $n_{e}$ of just $0.0057 \mathrm{~cm}^{-3}$, the Local spiral arm is not visible in Figure 10. Because of their extent and proximity to the Sun, a relatively large number of pulsars are affected by these local features.

The Local Bubble of course surrounds the Sun and so affects model distances for all pulsars. However, it only has a significant effect for those which are close to the Sun, say within 1 kpc. For PSR J0437-4715, not only does the entire path lie within the Local Bubble but also it has a very precisely measured distance. It clearly has a strong influence on the value of $J_{\mathrm{LB}}$ since the model distance is equal to the independent distance, both $156 \mathrm{pc}$. Of the seven pulsars with $D_{i} \leq 250 \mathrm{pc}$, five have distances based on parallax measurements and three of these are accurately modelled with $D_{\text {err }}=0$. The other two, PSRs J2144-3933 and J0633+1746, have model distances that are off by about a factor of two, PSR J2144-3933 over-estimated and PSR J0633+1746 under-estimated. The remaining two (PSRs J1745-3040 and J1752-2806) have kinematic distance estimates which are less precise; for the first, $D_{m}$ is about twice the upper limit of $D_{i}$, and for the second $D_{e r r}<20 \%$ but $D_{m}$ is at the upper limit of $D_{i}$ (Table 3). The Local Bubble is a significant feature of the model, with most of the local pulsars strongly affected by it having accurate model distances.

The associated density enhancements, LB1 and LB2, are relatively dense and, because of their size and proximity to the Sun, they significantly affect the model distances to a large number of pulsars lying at Galactic longitudes between about $150^{\circ}$ and $315^{\circ}$. Of the 55 pulsars with a significant DM contribution from LB1/2, 35 have satisfactorily modelled distances with $D_{\text {err }}<20 \%$, eight have over-estimated distances and 12 have under-estimated distances. Most of the over-estimated distances are for pulsars at negative latitudes, whereas most of the under-estimated distances are for pulsars at positive latitudes. This suggests that LB1/2 should be centered at $z<0$ rather than at $z=0$ as in the present model. However, at present we have insufficient data to quantify this offset. Most of the 35 well-modelled 


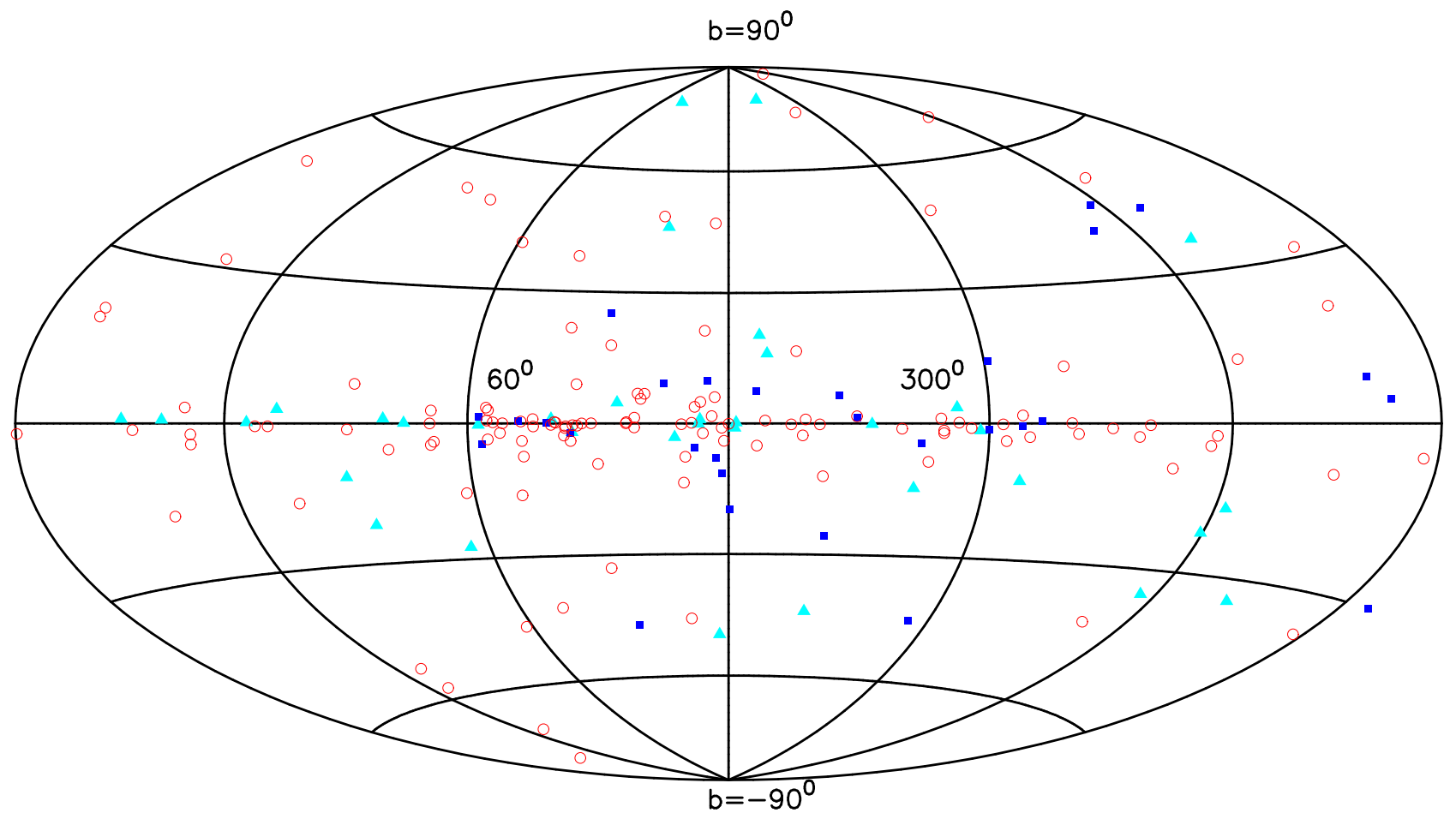

Figure 8. Positions of the 189 pulsars with model-independent distances plotted in Galactic coordinates. Pulsars with $D_{\text {err }}<$ $20 \%$ are plotted as red open circles, those with under-estimated distances as blue squares and those with over-estimated distances as green triangles.

distances would be over-estimated in the absence of LB1/2 and so these features are critical to the model.

As discussed above, the Gum Nebula shell has a relatively high density and makes a signficant contribution to the DM of pulsars within or behind it. Of the eight pulsars affected by the Gum Nebula, six have model distances within the independent-distance limits. The two exceptions, PSRs J0742-2822 and J0835-4510 (the Vela pulsar), have slightly over-estimated distances (Tables A2 and A1 respectively). Similarly, of the 13 pulsars having a DM contribution from Loop I (Figure 11), two (PSRs J1721-1936 and J1744-1134) have under-estimated distances (Table 4), one (PSR J1820-0427) has an over-estimated distance and the remaining ten have accurate model distances. Clearly, both the Gum Nebula and Loop I make important contributions to the model.

Table 3 shows that there are six pulsars for which the YMW16 model cannot account for the observed DM and therefore have nominal model distances of 25000 pc. Only one of these, PSR J0248+6021, discussed above in connection with Figure 7, is at low Galactic latitude; the remaining five are all have $|b| \gtrsim 30^{\circ}$. A further five pulsars in Table 3 also have $|b| \gtrsim 30^{\circ}$, but do have DMs within the model range. On the other hand, six of the 30 pulsars with under-estimated distances (Table 4) are also at high Galactic latitudes. These results show that the thick disk is well modelled on average, but that structure within it results in some over-estimated distances and some under-estimated distances.

Since nearly half of the 189 pulsars with independent distances have $D_{\text {err }}=0$, that is, a model distance within the uncertainty range of the independent distance, the distribution of $D_{\text {err }}$ is very asymmetric. The mean $D_{\text {err }}$ is $42 \%$ but the median is just $5 \%$. It is difficult to estimate the reliability of the model distances just using the known independent distances, since the model has been fitted to these distances. To overcome this problem, we adopt the following strategy:

1. Randomly select five pulsars from the list of 189 having independently determined distances

2. Do a full global plus local fit for model parameters as described in $\S 4$ on the remaining 184 pulsars

3. Use this model to compute distances $D_{m p}$ to the five omitted pulsars and store the results

4. Repeat steps 1-3 100 times, generating 500 "predicted" distances

5. Compute the relative distance error $\epsilon_{m p}=\left(D_{m p}-D_{i}\right) / D_{i}$ for each of these 500 predicted distances.

The left-top panel of Figure 12 shows the distribution of relative distance errors $\epsilon_{m}=\left(D_{m}-D_{i}\right) / D_{i}$ for all 189 pulsars used to detemine the YMW16 model. Similarly, the right-top panel shows the distribution of $\epsilon_{m p}$ for the 


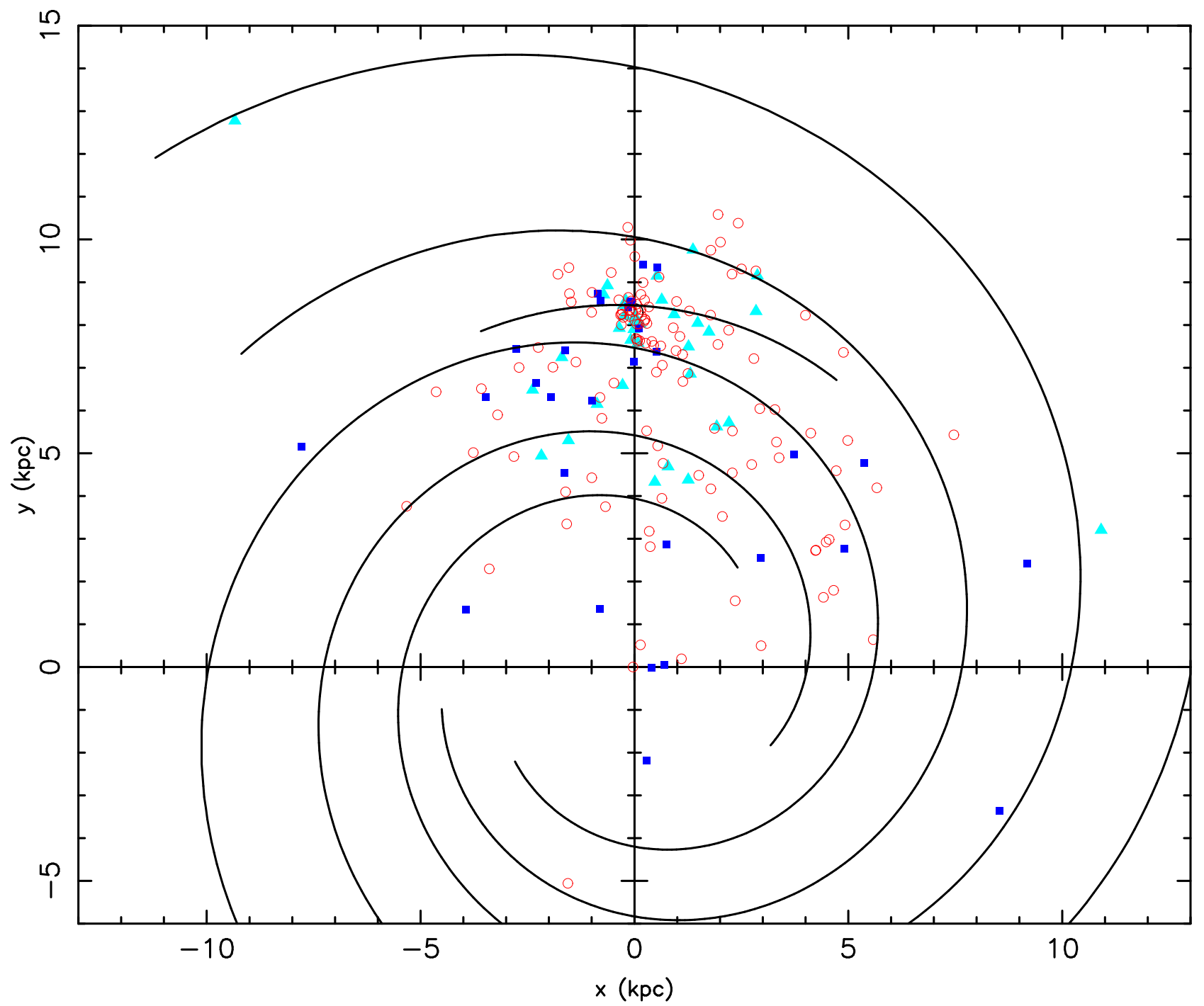

Figure 9. Positions of the 189 pulsars with model-independent distances projected onto the Galactic plane at the position of their independently estimated distance. Pulsars are plotted with the same symbols as in Figure 8.

above-described predicted distances. In both cases, there is a significant excess of over-estimated distances. For about half of this excess, $D_{m}$ (or $D_{m p}$ ) is at the limit, $25000 \mathrm{pc}$, for pulsars where the model cannot account for the observed DM. A Gaussian model has been fitted to both distributions with the $10 \%$ of pulsars having the largest absolute relative errors omitted from the fit in order to give a more "robust" estimate of the distribution parameters. Table 5 gives the results of this Gassian fitting showing that the rms deviation of $\epsilon_{m}$ is a little under $40 \%$ and for $\epsilon_{m p}$ about 43\%. Based on the latter, we conclude that the YMW16 model predicts pulsar distances based on their DM with a $95 \%$ confidence limit of approximately $90 \%$. That is, we estimate that $95 \%$ of all model predictions will have a relative error of less than a factor of 0.9 .

Table 5. Parameters of distance-error distributions

\begin{tabular}{lrcc}
\hline \hline \multicolumn{1}{c}{ Model } & Amp. & Mean & Rms devn \\
\hline YMW16 (189 dist.) & 81.5 & 0.012 & 0.398 \\
YMW16 (Pred. dist.) & 203.7 & 0.008 & 0.426 \\
NE2001 (189 dist.) & 60.7 & 0.060 & 0.571 \\
& & & \\
\hline
\end{tabular}

Table 5 continued on next page 


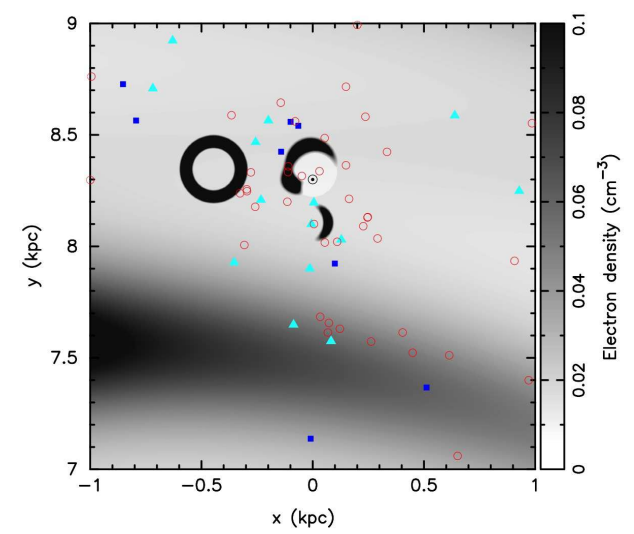

Figure 10. Pulsar positions projected onto the Galactic plane and $n_{e}$ distribution in the plane for the local region of the Galaxy. The position of the Sun is marked with $\odot$. Identifiable model features are the Carina enhancement and Carina - Sagittarius spiral arm, the Gum Nebula, the Local Bubble and surrounding density enhancements and Loop I (at $x, y=0.1,8.1 \mathrm{kpc}$ ). Pulsars are plotted with the same symbols as in Figure 8.



Figure 11. Pulsar positions in Galactic coordinates in the vicinity of Loop I, with the grey-scale showing the DM contribution of Loop I. Pulsars are plotted with the same symbols as in Figure 8.

Table 5 (continued)

\begin{tabular}{lrcc}
\hline \multicolumn{1}{c}{ Model } & Amp. & Mean & Rms devn \\
\hline NE2001 (Pred. dist.) & 21.7 & 0.033 & 0.599 \\
TC93 (189 dist.) & 41.0 & 0.284 & 0.856 \\
TC93 (Pred. dist.) & 14.8 & 0.303 & 0.887 \\
& & & \\
\hline
\end{tabular}

\subsection{Comparison of YMW16 with earlier Galactic $n_{e}$ models}

The most commonly used methods of estimating pulsar distances from their DM are based on the Galactic electron density models NE2001 and TC93. It is therefore of interest to compare the Galactic model and predictions of YMW16 with these earlier models and their predictions. All three models have the same basic structure for the Galactic $n_{e}$ with 

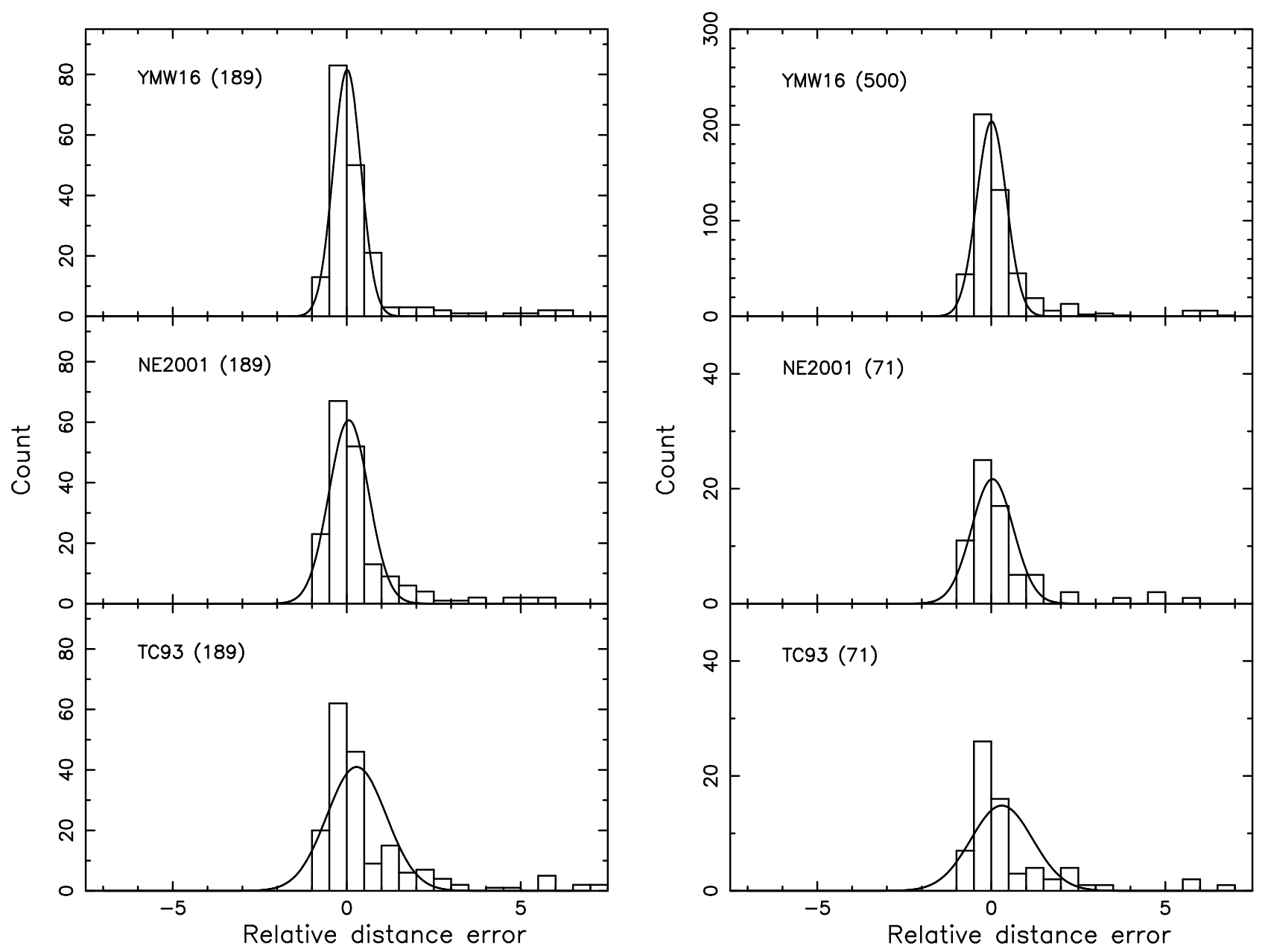

Figure 12. Histograms of relative distance errors $\left(D_{m}-D_{i}\right) / D_{i}$, where $D_{m}$ is the model distance based on the observed DM and $D_{i}$ is the independently determined distance, for three Galactic $n_{e}$ models: YMW16, NE2001 and TC93. Plots in the left column show the distributions for all 189 pulsars with independent distances, whereas plots in the right column show distributions for the "predictive" data sets. For all cases, the results of a "robust" fit of a Gaussian model to the distribution is shown. (See text for details.)

an extended thick disk, a thin disk largely confined to the inner Galaxy, spiral arms and a greater or lesser number of local features.

The model DM for high-latitude pulsars is primarily determined by the thick disk. Figure 13 shows the variation of $\mathrm{DM}_{\perp}=\mathrm{DM} \sin |b|$, with $|z|=D_{i} \sin |b|$, where $b$ is the Galactic latitude, for the 189 pulsars with independent distances. Curves giving the $\mathrm{DM}_{\perp}$ contribution of the thick-disk component for other Galactic $n_{e}$ models are also shown. For pulsars at low latitudes, there is large $\mathrm{DM}_{\perp}$ contribution from other components of the Galactic $n_{e}$ distribution, especially the spiral arms and the thin disk. However, even for high-latitude pulsars, there is some contribution from these other components. Figure 13 shows the effect of subtracting these other components for the YMW16 model, to give the thick-disk component alone.

Recent models, including YMW16, have largely converged on a larger scale height $\sim 1600 \mathrm{pc}$ and a lower $\mathrm{DM}_{\perp} \sim$ $20 \mathrm{~cm}^{-3}$ pc for the thick disk. For NE2001, the thick disk clearly includes a much larger contribution from low- $z$ components that are assigned to spiral arms or the thin disk in other models. As discussed in $\S 1$, it also substantially over-estimates the high- $z$ DMs or, equivalently, under-estimates the distances of high $z$ pulsars which are mostly at high $|b|$. It is notable that this is not the case for the earlier TC93 model.

The thin disk is very similar in YMW16, NE2001 and TC93, an annulus with radius about 4 kpc, although for YMW16 the disk scale height is about 65 pc compared to 150 pc for TC93 and 140 pc for NE2001.

For the spiral arms, YMW16 adopts a simple logarithmic-spiral pattern based on HII-region distances (Hou \& Han 2014) in contrast to the somewhat more complex modified spiral pattern used by TC93 and NE2001. Part of the modified pattern used by these earlier models was to reduce $n_{e}$ in the nominal Sagittarius tangential region. In 


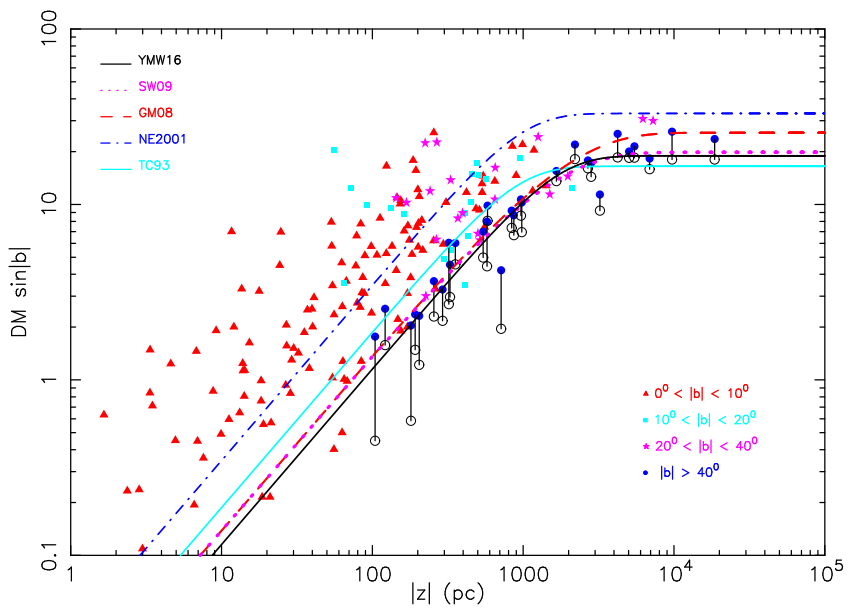

Figure 13. $\mathrm{DM}_{\perp}=\mathrm{DM} \sin |b|$ versus $|z|=D_{i} \sin |b|$ for Galactic pulsars. The lines show the variation of $\mathrm{DM}_{\perp}$ for the thickdisk component of several different models of the Galactic $n_{e}$ distribution. For SW09 (Savage \& Wakker 2009) and GM08 (Gaensler et al. 2008) the line is based on fits of just the thick disk to DMs and distances for high-latitude pulsars, but for TC93, NE2001 and YMW16, the line represents the thick-disk component resulting from a global fit to the distance data. The thick-disk DM components for the YMW16 model are shown by open circles for the high-latitude pulsars.

YMW16, this is accomplished by defining an under-dense zone in the Sagittarius arm. An over-dense zone in the Carina arm is also included in YMW16.

TC93 has just one local feature, the Gum Nebula, whereas NE2001 has 82 local clumps, including the Gum Nebula and a clump surrounding the Vela pulsar, 17 voids and four other local features including the Local Bubble and Loop I. As described in $\S 3$ and $\S 5$ above, YMW16 takes a more conservative approach with just seven local features chosen on the basis that each affected the model distance for a group of pulsars either within or behind the feature. The parameters of these features are based on relatively recent studies unavailable to both TC93 and NE2001.

Finally, YMW16 includes components representing the Magellanic Clouds and the intergalactic medium. These components were not included in any previous model for pulsar distance estimation.

Comparisons of the YMW16 model predictions with those of TC93 and NE2001 are difficult since these earlier models were based on a smaller sample of independent distances (74 pulsars for TC93 and 112 for NE2001) than that used for our model. However, for the moment, we will ignore this issue and directly compare the distance predictions of the YMW16 model with those of earlier models for the currently known 189 pulsars with independent distance data.

In Table 6 we summarise the distribution of distance errors $D_{\text {err }}$ for the YMW16, NE2001 and TC93 models. It is clear that the YMW16 model has benefited greatly from recent parallax measurements for relatively high-latitude pulsars from VLBI (e.g., Chatterjee et al. 2009) and pulsar timing array projects (Reardon et al. 2016; Matthews et al. 2016). These have allowed a much better definition of the thick disk and consequently smaller distance errors for highlatitude pulsars with 19 of the 29 high-latitude pulsars having $D_{\text {err }}<20 \%$. Overall, the YMW16 model has significantly smaller distance errors than NE2001 and much smaller distance errors than TC93.

Table 6. Comparison of distance errors with previous models

\begin{tabular}{|c|c|c|c|c|c|c|c|c|}
\hline \multirow[b]{2}{*}{ Model } & \multicolumn{4}{|c|}{29 pulsars with $|b|>40^{\circ}$} & \multicolumn{4}{|c|}{189 pulsars } \\
\hline & $0 \%$ & $0 \%-20 \%$ & $20 \%-40 \%$ & $>40 \%$ & $0 \%$ & $0 \%-20 \%$ & $20 \%-40 \%$ & $>40 \%$ \\
\hline YMW16 & 14 & 5 & 4 & 6 & 86 & 38 & 25 & 40 \\
\hline NE2001 & 6 & 5 & 3 & 15 & 77 & 32 & 19 & 61 \\
\hline TC93 & 6 & 5 & 4 & 14 & 45 & 36 & 29 & 79 \\
\hline
\end{tabular}

In Figure 14 we directly compare the model and independent distances for the YMW16, NE2001 and TC93 models. 
We also show the results of weighted least-square fits of a linear relationship

$$
\log D_{m}=a \log D_{i}+b
$$

with weights equal to $1 /\left(\log D_{u}-\log D_{i}\right)$. Table 7 gives the results of the fits and also the correlation coefficients computed using the same weights. Not surprisingly, the YMW16 model is a better fit to the independent distance data and has a higher correlation coefficient with it. Compared to the earlier models, many fewer distances are overestimated. However, these over-estimated distances generally have large distance undertainties. In fact, more of the high-weight distances are under-estimated by the earlier models, resulting in fitted lines of smaller slope located largely below the line of equality. Somewhat surprisingly though, the TC93 model has both a slope closer to 1.0 and a higher correlation coefficient compared to the NE2001 model.

Table 7. Distance model fits

\begin{tabular}{lccc}
\hline \hline & \multicolumn{2}{c}{ Least-square fits } & Correlation \\
Model & $a$ & $b$ & coefficient \\
\hline YMW16 & 0.946 & 0.104 & 0.932 \\
NE2001 & 0.809 & 0.495 & 0.877 \\
TC93 & 0.894 & 0.239 & 0.892 \\
\hline
\end{tabular}

In $\S 5$ above, we estimated the reliability of the YMW16 model by repeatedly fitting the independent distance data to 184 pulsars randomly chosen from the 189 with independent distances currently available and then comparing model and independent distances for the omitted five pulsars. For NE2001 and TC93, the process is simpler, since many independent distances have been published since these models were created. Unfortunately, however, neither Taylor \& Cordes (1993) nor Cordes \& Lazio (2002) or Cordes \& Lazio (2003) published the list of independent distances used in constructing their model. We have therefore selected the set of 71 independent distance measurements published since 2008 in order to test the predictive abilities of these models. ${ }^{13}$ Distributions of $\epsilon_{m}$ and $\epsilon_{m p}$ are shown in Figure 12 and the results of the robust Gaussian fitting are given in Table 5. Unsurprisingly, the two earlier models are significantly inferior to YMW16 when tested against the full sample of 189 independent distances. However, this inferior performance also carries over to the "predictive" data sets. For NE2001 and TC93, the rms deviations $\epsilon_{m p}$ are about $60 \%$ and $89 \%$ respectively, corresponding to $95 \%$ confidence limits of approximately $120 \%$ and $180 \%$ for the two models.

Differences in the performance of the three models are also clearly illustrated by cumulative histograms. Following Schnitzeler (2012), we have used distance ratios, $\rho_{m}$, where $\rho_{m}=D_{m} / D_{i}$ for $D_{m} / D_{i} \geq 1.0$ and $\rho_{m}=D_{i} / D_{m}$ for $D_{m} / D_{i}<1.0$, rather than the relative distance errors used for the histograms in Figure 12, as this allows use of a $\operatorname{logarithmic} x$ axis for the cumulative histograms, and similarly for $\rho_{m p}$. Figure 15 shows these cumulative histograms for the three models. For both the current and predictive data sets, the superior performance of the YMW16 model is evident. Both Figure 12 and Figure 15 show that TC93 signficantly over-estimates many distances. Figure 15b shows that, for low values of $\rho_{m p}$ i.e. accurately predicted distances, despite the much smaller number of free parameters in the YMW16 model, but YMW16 does much better for larger $\rho_{m p}$.

\subsection{Interstellar scattering}

Predicted interstellar scattering times at 1.0 GHz, based on the Krishnakumar et al. (2015) model (Equation 36) for Galactic pulsars with independent distances are given in Tables A1 - A5. The Krishnakumar et al. (2015) model was based on a fit to a total of 358 measurements of scattering times for Galactic pulsars. In Figure 16 we plot the ratios of model predictions to observed scattering times, scaled to $1.0 \mathrm{GHz}$ assuming $\tau_{\mathrm{sc}} \propto \nu^{-4.0}$, for YMW16 and NE2001. In addition to $354 \tau_{\mathrm{sc}}$ values and corresponding observation frequencies provided to us by M. Krishnakumar (private communication), we include the recently measured scattering timescale for the Galactic Center pulsar, PSR

13 Despite the TC93 and NE2001 models using 74 and 112 independent distances respectively, the ATNF Pulsar Catalogue and Verbiest et al. (2012) show that only 41 distances were formally published before 1993 and 90 before 2002. We have therefore selected a cutoff date that gives somewhat less than $189-112=77$ distances. It is likely that some unpublished distances were used in constructing these models and also that updated distance estimates have been published for some pulsars. 




Figure 14. Distances from the YMW16, NE2001 and TC93 Galactic $n_{e}$ models versus independently measured distances and uncertainties for the 189 pulsars where these are known. The sloping black lines correspond to $D_{m}=D_{i}$ and the red dashed lines show the result of a weighted least-squares fit of $\log D_{m}$ as a function of $\log D_{i}$.
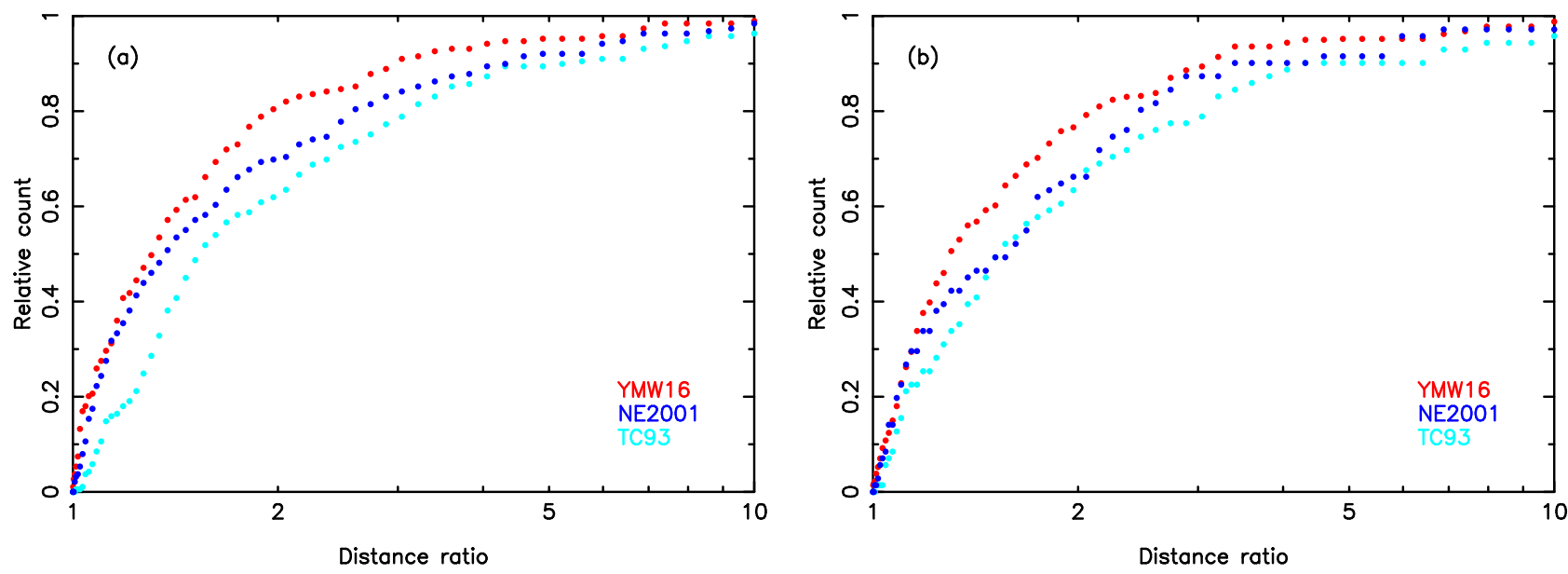

Figure 15. Cumulative histograms of distance ratios (a) $\rho_{m}$ and (b) $\rho_{m p}$ for the full sample of 189 independently determined distances and the predictive data sets, respectively, for the three Galactic $n_{e}$ models: YMW16, NE2001 and TC93. (See text for details.)

J1745-2900, which has a DM $=1778 \mathrm{~cm}^{-3}$ pc (Eatough et al. 2013) in this figure. Pennucci et al. (2015) measured $\tau_{\mathrm{sc}}=0.1330 \pm 0.0005 \mathrm{~s}$ at $2.0 \mathrm{GHz}$ and a frequency index $\alpha=-3.71 \pm 0.02$ for the scattering timescale of PSR J1745-2900. Scaling this to $1 \mathrm{GHz}$ assuming an index of -4.0 gives $\tau_{\mathrm{sc}}=2.13 \mathrm{~s}$, whereas an index of -3.71 gives $1.74 \mathrm{~s}$. Both of these are close to the YMW16 prediction of $3.55 \mathrm{~s}$ (cf. Table A4). For consistency, we have chosen 
to plot the former value. As discussed by Pennucci et al. (2015), there is some indication of time variability in the scattering timescale for this pulsar, with Spitler et al. (2014b) measuring $\tau_{\mathrm{sc}}=1.3 \pm 0.2 \mathrm{~s}$ at $1 \mathrm{GHz}$.

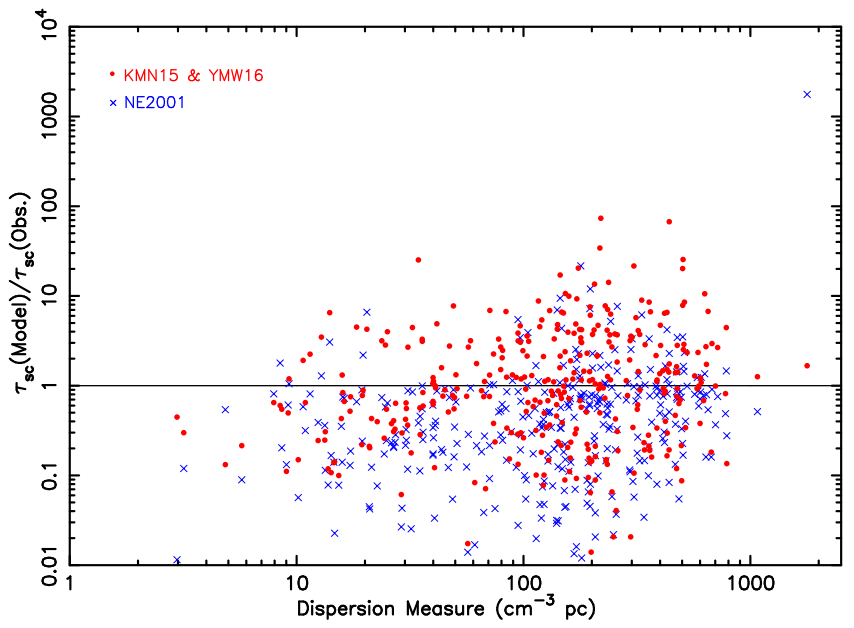

Figure 16. Ratio of model scattering times to observed scattering times, normalized to $1 \mathrm{GHz}$, for $355 \mathrm{Galactic}$ pulsars as a function of dispersion measure. The red dots are for the YMW16 model which is based on the results of Krishnakumar et al. (2015) and the blue crosses are for the predictions of the NE2001 model.

As expected, Figure 16 shows the KMN15/YMW16 points essentially uniformly distributed about $R_{\mathrm{sc}}=\tau_{\mathrm{sc}}($ Model $) / \tau_{\mathrm{sc}}($ Obs. $)$ $=1$ line. However, with the exception of the PSR J1745-2900 point, the NE2001 values are systematically biased low. For KMN15/YMW16, the mean and rms deviation of $\log R_{\mathrm{sc}}$ (excluding PSR J1745-2900) are $-0.080 \pm 0.039$ and 0.735, respectively, whereas for NE2001 the corresponding values are $-0.573 \pm 0.046$ and 0.863 . For NE2001, the mean offset in the log corresponds to $\tau_{\mathrm{sc}}$ being low on average by a factor of $\sim 3.7$. Different assumptions about scaling from observations at other frequencies may influence these results, but it is very unlikely that they can account for the general under-estimation of $\tau_{\mathrm{sc}}$ by NE2001.

The NE2001 model predicts a very large scattering timescale for pulsars within or behind the Galactic Center disk and, in particular for PSR J1745-2900 predicts $\tau_{\mathrm{sc}}=3750 \mathrm{~s}$, about a factor of 1800 greater than the observed value. Bower et al. (2014) argue that the principal scattering screen for PSR J1745-2900 is located about $5.9 \mathrm{kpc}$ from the Galactic Center, in the Scutum spiral arm, rather than in the Galactic Center region as assumed by Cordes \& Lazio (2002). The close agreement of the observed $\tau_{\text {sc }}$ for this pulsar with the prediction of the Krishnakumar et al. (2015) model for Galactic scattering shows that the scattering observed for this pulsar is not exceptional given its large DM and hence supports the conclusions of Bower et al. (2014).

\subsection{The Magellanic Clouds}

The location, size and shape of the Large and Small Magellanic Clouds are based primarily on the distribution of young stars and star-forming regions in the Clouds. The models are relatively simple as the number of pulsars known to be associated with the Clouds is small (Table A6) and, in most cases, we have no prior information on the location of individual pulsars within the Clouds. For the LMC, the derived central electron density is $0.066 \mathrm{~cm}^{-3}$, comparable to the electron density averaged over the Galactic disk. The derived central electron density of the separately-modelled giant HII region 30 Doradus is about $0.3 \mathrm{~cm}^{-3}$, but this is quite uncertain as it is primarly determined by one pulsar (PSR J0537-69) and we don't know the line-of-sight position of this pulsar relative to the nebula.

Of the five pulsars associated with the SMC (Table A6), four have relatively small DMs $\left(<100 \mathrm{~cm}^{-3} \mathrm{pc}\right)$. The fifth, PSR J0131-7310, has a DM of $175 \mathrm{~cm}^{-3}$ pc despite being located right at the edge of the SMC. The diffuse H $\alpha$ survey of Barger et al. (2013) shows a tongue of emission covering the position of PSR J0131-7310 and the HII region N90 (Lawton et al. 2010) is nearby. The SMC images in the Barbara A. Milulski Archive for Space Telescopes ${ }^{14}$ show an $\mathrm{H} \alpha$ shell surrounding the pulsar. There is no significant radio continuum feature at the pulsar position in the $4.8 \mathrm{GHz}$ image of Lawton et al. (2010). It is possible that this pulsar just happens to lie behind a relatively high-density clump of ionised gas, possibly part of the surrounding $\mathrm{H} \alpha$ shell. However, we choose not to explicity model this and omit

14 https://archive.stsci.edu/prepds/fuse_mc/overviewimages.html 
this pulsar from the SMC fit in order to give a more representative model for the SMC $n_{e}$ distribution. The derived central density for the SMC is $0.045 \mathrm{~cm}^{-3}$ (Table 2).

Model distances for the Magellanic Cloud pulsars are given in Table A6 along with the DM contributions of the Galaxy and Magellanic Clouds. Because of the relatively high Galactic latitudes of the Magellanic Clouds, the Galactic contributions to the DM are usually small compared to the Magellanic Cloud contributions. However, there are exceptions, in particular, for PSR J0451-67. The discovery paper for this pulsar (Manchester et al. 2006) recognised its uncertain association with the LMC, and we confirm that here. For NE2001, the Galactic model DM in this direction is $\sim 2 \mathrm{~cm}^{-3} \mathrm{pc}$ less than the observed DM $\left(45 \pm 1 \mathrm{~cm}^{-3} \mathrm{pc}\right)$ putting the pulsar in the LMC, but for YMW16, the Galactic model DM $\left(48.3 \mathrm{~cm}^{-3} \mathrm{pc}\right)$ is about $3 \mathrm{~cm}^{-3} \mathrm{pc}$ more than the observed DM, giving the pulsar an unrealistic model distance of $\sim 4.2 \mathrm{kpc}$ (Table A6). Future revisions of the distance model and/or better determination of the pulsar parameters may resolve this uncertainty about the association. For several other nominally LMC pulsars, $\mathrm{DM}_{\mathrm{Gal}}$ dominates the observed DM, but the association is likely to be correct in these cases. The total model DM in the direction of the SMC pulsar PSR J0131-7310, discussed above, is about $85 \mathrm{~cm}^{-3} \mathrm{pc}$, much less than the pulsar DM of $205 \mathrm{~cm}^{-3} \mathrm{pc}$, and so the model distance is just a nominal upper limit.

Predicted scattering timescales for the Magellanic Cloud pulsars are listed in Table A6. For 20 of the 27 pulsars listed, the overall scattering time is dominated by the scattering occuring in the Magellanic Clouds. The largest predicted scattering timescale is $0.27 \mathrm{~ms}$ for PSR J0537-69. This pulsar has a relatively short period of $112 \mathrm{~ms}$. Along with the rest of the Magellanic Cloud pulsars, PSR J0537-69 is relatively weak and its best profile (Ridley et al. 2013) has insufficient sensitivity to detect a scattering tail. In fact, no Magellanic Cloud pulsars have measured scattering timescales.

\subsection{Fast Radio Bursts}

Table A7 gives the model redshift and co-moving distance estimates for the 17 currently known FRBs based on the model of Katz (2016) which assumes a present-day baryon density $n_{\mathrm{IGM}}=0.16 \mathrm{~m}^{-3}$. Model Galactic, Magellanic Cloud and IGM contributions to the DM are listed in Table A7. As discussed in $\S 3.10$, by default, we adopt a value of $100 \mathrm{~cm}^{-3} \mathrm{pc}$ for $\mathrm{DM}_{\text {Host }}$, the observed $(z=0)$ DM contribution of the FRB host galaxy. Derived redshifts range between 0.2 and 2.1 and model distances from $900 \mathrm{Mpc}$ to 5 Gpc. Several aspects of these results are worth further comment.

For the first time, we estimate the contribution of the SMC to the DM of the original Lorimer Burst (Lorimer et al. 2007), FRB010724, obtaining $\mathrm{DM}_{\mathrm{MC}} \sim 61 \mathrm{~cm}^{-3}$ pc. Lorimer et al. (2007) assumed $\mathrm{DM}_{\mathrm{Host}}=200 \mathrm{~cm}^{-3} \mathrm{pc}$ and a distance of $500 \mathrm{Mpc}$ and hence a redshift $z \sim 0.12$. The YMW16 model gives a redshift of 0.254 and a co-moving distance of 1 Gpc. However, as discussed in $§ 5.4$, the SMC model is based on just four pulsars and omits one high-DM pulsar, PSR J0131-7310, so it is quite possible that our estimate of $\mathrm{DM}_{\mathrm{MC}}$ in this direction is under-estimated. In any case, it is clear that the SMC contribution to the DM of FRB010724 is significant.

For FRB150418, the only FRB with a (possible) identification (Keane et al. 2016), with no fine tuning whatsoever, the YMW16 model redshift is 0.492 (Table A7), exactly the redshift of the suggested galaxy identification. While it is interesting that the model redshift is close to the galaxy redshift, the exact match is no more than a remarkable coincidence. This is illustrated by the derived redshifts of 0.562 and 0.351 for $\mathrm{DM}_{\mathrm{Host}}$ equal to $50 \mathrm{~cm}^{-3} \mathrm{pc}$ and $200 \mathrm{~cm}^{-3}$ pc, respectively.

In the discovery paper, Spitler et al. (2014a) discuss the origin of FRB121102 and conclude that it is most likely to be extra-galactic. This choice was primarily based on the NE2001 estimate of the Galactic contribution, $188 \mathrm{~cm}^{-3} \mathrm{pc}$ being a small fraction of the total DM, $557 \mathrm{~cm}^{-3}$ pc. However, as Table A7 shows, the YMW16 model gives DM Gal $_{2} \sim$ $287 \mathrm{~cm}^{-3} \mathrm{pc}$, more than $50 \%$ of the total DM. While the residual DM is still relatively large, this new result does somewhat weaken the argument that the source is extragalactic. Furthermore, FRB121102 is the only FRB known to emit multiple pulses (Spitler et al. 2016). This also marks it as unusual since no repeating pulses have been found from other FRBs (Petroff et al. 2015b).

Both of these points lead one to consider the relationship of FRBs to RRATs. RRATs are radio sources that emit detectable pulses only sporadically (McLaughlin et al. 2006b). In most cases, their DMs suggest that they are Galactic sources and careful analyses of pulse arrival times have revealed periodicties within the range of pulsar periods (e.g., McLaughlin et al. 2006b; Karako-Argaman et al. 2015). Consequently, they are generally considered to be a class of Galactic pulsars with unusual emission properties. However, the "RRATalog" 15 , a list of currently known RRATs,

15 http://astro.phys.wvu.edu/rratalog/ 
lists 12 sources where only a single pulse has been detected. Several of these have DMs that are close to the estimated total Galactic DM in their direction, notably J1059-01, J1354+24 and J1610-17, which have DM/DM Gal,total ratios of $0.711,0.975$ and 0.815 , respectively. It is possible that these sources and maybe a few others could in fact be extragalactic FRBs (cf., Keane 2016). Conversely, the properties of FRB121102 suggest that it could be a Galactic RRAT, a possibilty that was indeed considered by Spitler et al. (2014a). Several other FRBs have low Galactic latitudes and large estimated $\mathrm{DM}_{\mathrm{Gal}}$ values (Table A7) leading to doubt about their extra-galactic origin (e.g., Bannister \& Madsen 2014). Given the uncertainties inherent in Galactic $n_{e}$ models, the identification of burst sources with DMs near the total Galactic DM in their direction as Galactic or extra-galactic will remain problematic. Consideration of other properties such as pulse shape and whether or not there are repeated pulses may necessary to discriminate between Galactic and extra-galactic origins for observed burst sources.

Predicted scattering timescales for FRBs are listed in the final two columns of Table A7. All but three of these are dominated by the IGM contribution. For the exceptions, FRB010621, FRB121102 and FRB150418, the observed scattering times are upper limits and the predicted Galactic scattering time is less than the upper limit. Consequently, these cases do not significantly affect our FRB scattering model (Equation 42). FRB130729 stands out with the observed scattering timescale (Champion et al. 2016) much larger than is predicted by the model. The observed profile for this FRB has a rather low signal-to-noise ratio and it is possible that it has an intrinsic double-peaked profile similar to FRB121002 (Champion et al. 2016). In this case the estimate of the scattering timescale would be much reduced.

\section{SUMMARY AND CONCLUSIONS}

In this paper we have constructed a new model, YMW16, for the distribution of thermal free electrons in the Galaxy with the main aim of providing more reliable distance estimates for real or simulated pulsars based on their dispersion measure. The model also includes contributions from the Magellanic Clouds and the intergalactic medium (IGM), enabling distance estimates for extra-galactic pulsars and FRBs. The Galactic model is based on independently determined distances for 189 pulsars. As tabulated in Appendix A, these model-indepependent distances are derived from measurements of annual parallax, HI absorption together with a kinematic model for Galactic rotation, associations with globular clusters, supernova remnants or (in one case) an HII region or distance estimates for binary companion stars. While we estimate the amount of pulse broadening due to interstellar scattering for Galactic and Magellanic Cloud pulsars and FRBs, we do not use scattering data as an input to the model.

The main components of the model are as follows: a plane parallel thick disk with a scale height of about $1600 \mathrm{pc}, \mathrm{a}$ thin disk of scale height about 65 pc mainly representing the "molecular ring" of the Galaxy around 4 kpc Galactocentric radius, spiral arms following a logarithmic spiral pattern based on the Galactic distribution of HII regions (Hou \& Han 2014), a Galactic Center disk of radius 160 pc and scale height 35 pc, an ellipsoidal shell with major axis in the $z$ direction representing the Gum Nebula, the Local Bubble, a region of reduced $n_{e}$ surrounding the Sun, together with regions of enhanced density on the periphery of the Bubble, and a hemispherical shell of enhanced density associated with the nearby Galactic Loop I. The Carina-Sagittarius spiral arm has a region of enhanced $n_{e}$ on the Carina side and a region of depressed $n_{e}$ on the Sagittarius side. All Galactic disk components follow a $\operatorname{sech}^{2}(z / H) z$-dependence, where $H$ is the scale height, and have a $\operatorname{sech}^{2}(R)$ cutoff at $R=15 \mathrm{kpc}$ with a scale length of $2.5 \mathrm{kpc}$. Unlike the NE2001 model, the YMW16 model does not have clumps or voids toward particular pulsars or highly-scattered galactic or extra-galactic sources. The $n_{e}$ distribution in the Magellanic Clouds is based on the distribution of star-formation regions and young stars. The SMC is modelled as a spherical distribution and the LMC as an inclined disk with an additional component representing the giant HII region 30 Doradus. The IGM model is based on the relations given by Katz (2016) with a mean present-day intergalactic $n_{e}$ of $0.16 \mathrm{~m}^{-3}$.

In total, the YMW16 model has 82 fixed parameters and 35 fitted parameters. A non-linear least-squares procedure was used to fit the 32 free parameters of the Galactic model to the 189 independently measured pulsar distances. The three free parameters of the Magellanic Cloud model were separately fitted to the 27 Magellanic Cloud pulsars with known DMs. Values of the fixed and fitted parameters and their uncertainties are given in Section 3 and Table 2 and the overall form of the model is shown in Figure 4. Of the 189 Galactic pulsars, the model distance is within the uncertainties of the independent distance for 86 pulsars and within $20 \%$ of the nearest limit for a further 38 pulsars. By repeatedly selecting a random sample containing 184 of the 189 pulsars, refitting the model to these 184 pulsars and checking the model prediction for the remaining five pulsars, we estimate that $95 \%$ of model predictions will have a relative error of less than $90 \%$.

Comparison of the YMW16 model distances with those of the TC93 and NE2001 models for the full sample of 189 pulsars showed that the new model performs substantially better, especially for high-latitude pulsars. However, this 
is not a fair comparison as we have the advantage of fitting the full 189-pulsar sample whereas TC93 and NE2001 did not. For a fairer comparison we compared the model predictions from the repeated fitting the randomly chosen 184pulsar sample with predictions of the TC93 and NE2001 models for a sample of 71 pulsars with recently determined independent distances. This showed that YMW16 is expected to perform about 50\% better than NE2001 and 100\% better than TC93 in estimating pulsar distances based on their DM.

While the YMW16 model performs significantly better than earlier models in predicting the distances of pulsars based on their DM, there is certainly room for improvement. At present less than $10 \%$ of known pulsars have independently determined distances and only about $8 \%$ have sufficiently accurate distance estimates to be useful. More than $75 \%$ of these are located within $5 \mathrm{kpc}$ of the Sun. New relatively precise independent distance estimates, especially for more distant pulsars, will enable us both to better test the model and to improve it. Currently the model contains 15 components describing the $n_{e}$ distribution in the Galaxy, including five spiral arms and seven relatively local features such as the Carina excess and the Gum Nebula. The highly anomalous prediction for PSR J0248+6021 highlights the effect of HII regions on the predicted distances. YMW16 includes the several relatively local over-dense regions, but does not attempt to model more distant HII regions such as W5. A future iteration of the model would benefit from inclusion of at least the larger and denser HII regions within a few kpc of the Sun. It is however impossible to model the detailed structure of the ISM throughout the Galaxy or even within the relatively local region, and so it is inevitable that there will be errors and uncertainties in the predicted distances.

The YMW16 model is the first to include models (albeit relatively simple models) for the $n_{e}$ distribution in the Large and Small Magellanic Clouds and for the intergalactic medium. These provide useful information on the expected DMs and distances for extra-galactic pulsars and redshift and distance estimates for known and hypothetical FRBs. Notable among the results is the significant $\mathrm{DM}_{\mathrm{MC}}$ for the Lorimer Burst.

We thank Bill Coles for help with the definition of the goodness-of-fit statistic, for suggesting the adopted method of estimating parameter uncertainties and for useful discussions on the effects of interstellar scattering, and M. Krishnakumar for providing us with tables of scattering data for Galactic pulsars. We also thank Matthew Bailes, Simon Johnston, JP Marquart and Ryan Shannon for useful discussions and George Hobbs and Hailong Zhang for help with the YMW16 web interface. Finally, we thank the referee for helpful comments. This work was supported by National Basic Research Program of China (973 Program 2015CB857100 and 2012CB821801), the Strategic Priority Research Programme (B) of the Chinese Academy of Science (No. XDB09000000), the National Natural Science Foundation of China (No. 11373006) and West Light Foundation of CAS (No. ZD201302). We acknowledge extensive use of the SAO/NASA Astrophysics Data System (http://www.adsabs.harvard.edu/) in the preparation of the work presented here.

\section{REFERENCES}

Akiyama, K., \& Johnson, M. D. 2016, ApJ, 824, L3

Alves, M. I. R., Calabretta, M., Davies, R. D., et al. 2015, MNRAS, 450, 2025

Anderson, L. D., Armentrout, W. P., Johnstone, B. M., et al. 2015, ApJS, 221, 26

Bailey, M., van Loon, J. T., Farhang, A., et al. 2016, A\&A, 585, A12

Bannister, K. W., \& Madsen, G. J. 2014, MNRAS, 440, 353

Barger, K. A., Haffner, L. M., \& Bland-Hawthorn, J. 2013, ApJ, 771,132

Bell, J. F., \& Bailes, M. 1996, ApJ, 456, L33

Berkhuijsen, E. M., Haslam, C. G. T., \& Salter, C. J. 1971, A\&A, 14, 252

Berkhuijsen, E. M., Mitra, D., \& Mueller, P. 2006, Astron. Nachrichten, 327, 82

Bland-Hawthorn, J., \& Cohen, M. 2003, ApJ, 582, 246

Bower, G. C., Deller, A., Demorest, P., et al. 2014, ApJ, 780, L2

—. 2015, ApJ, 798, 120

Brisken, W. F., Macquart, J.-P., Gao, J. J., et al. 2010, ApJ, 708, 232

Brunthaler, A., Reid, M. J., Menten, K. M., et al. 2011, Astron. Nachrichten, 332, 461

Burke-Spolaor, S., \& Bannister, K. W. 2014, ApJ, 792, 19
Caleb, M., Flynn, C., Bailes, M., et al. 2016, MNRAS, 458, 708

Champion, D. J., Petroff, E., Kramer, M., et al. 2016, MNRAS, 460, L30

Chatterjee, S., Brisken, W. F., Vlemmings, W. H. T., et al. 2009, ApJ, 698, 250

Cordes, J. M., \& Lazio, T. J. W. 2002, arXiv:astro-ph/0207156

Cordes, J. M., \& Lazio, T. J. W. 2003, arXiv:astro-ph/0301598

Cordes, J. M., Weisberg, J. M., Frail, D. A., Spangler, S. R., \& Ryan, M. 1991, Nature, 354, 121

Cordes, J. M., Wharton, R. S., Spitler, L. G., Chatterjee, S., \& Wasserman, I. 2016, arXiv:1605.05890

Crocker, R. M., Bicknell, G. V., Taylor, A. M., \& Carretti, E. 2015, ApJ, 808, 107

da Silva Santos, C. H., Gonçalves, M. S., \& Hernandez-Figueroa, H. E. 2010, IEEE Photonics Tech. Lett., 22, 1177

Eatough, R. P., Falcke, H., Karuppusamy, R., et al. 2013, Nature, 501, 391

Efron, B. 1981, Biometrika, 68, 589

Finkbeiner, D. P. 2003, ApJS, 146, 407

Frail, D. A., \& Weisberg, J. M. 1990, AJ, 100, 743

Gaensler, B. M., Madsen, G. J., Chatterjee, S., \& Mao, S. A. 2008, PASA, 25, 184 
Gaustad, J. E., McCullough, P. R., Rosing, W., \& Van Buren, D. 2001, PASP, 113, 1326

Genzel, R., Eisenhauer, F., \& Gillessen, S. 2010, Rev. Modern Physics, 82, 3121

Georgelin, Y. M., \& Georgelin, Y. P. 1976, A\&A, 49, 57

Gómez, G. C., Benjamin, R. A., \& Cox, D. P. 2001, AJ, 122, 908

Grabelsky, D. A., Cohen, R. S., Bronfman, L., \& Thaddeus, P. 1988, ApJ, 331, 181

Green, J. A., Caswell, J. L., McClure-Griffiths, N. M., et al. 2011, ApJ, 733, 27

Gum, C. S. 1952, Observatory, 72, 151

Haffner, L. M., Reynolds, R. J., \& Tufte, S. L. 1999, ApJ, 523, 223

Haffner, L. M., Reynolds, R. J., Tufte, S. L., et al. 2003, ApJS, 149,405

Han, J. L., Manchester, R. N., Lyne, A. G., Qiao, G. J., \& van Straten, W. 2006, ApJ, 642, 868

Handley, W. J., Hobson, M. P., \& Lasenby, A. N. 2015, MNRAS, 453, 4384

Haschke, R., Grebel, E. K., \& Duffau, S. 2012a, AJ, 144, 106

—. 2012b, AJ, 144, 107

Hill, A. S., Benjamin, R. A., Haffner, L. M., Gostisha, M. C., \& Barger, K. A. 2014, ApJ, 787, 106

Hill, A. S., Benjamin, R. A., Kowal, G., et al. 2008, ApJ, 686, 363

Hou, L. G., \& Han, J. L. 2014, A\&A, 569, A125

Hoyle, F., \& Ellis, G. R. A. 1963, Australian Journal of Physics, 16,1

Hughes, A., Staveley-Smith, L., Kim, S., Wolleben, M., \& Filipović, M. 2007, MNRAS, 382, 543

Johnston, S., Lyne, A. G., Manchester, R. N., et al. 1992, MNRAS, 255, 401

Joshi, Y. C., Dambis, A., Pandey, A. K., \& Joshi, S. 2016, arXiv: 1606.06425

Kalberla, P. M. W., Dedes, L., Kerp, J., \& Haud, U. 2007, A\&A, 469, 511

Kalberla, P. M. W., \& Kerp, J. 2009, Ann. Rev. Astr. Ap., 47, 27

Karako-Argaman, C., Kaspi, V. M., Lynch, R. S., et al. 2015, ApJ, 809, 67

Katz, J. I. 2016, ApJ, 818, 19

Keane, E. F. 2016, MNRAS, 459, 1360

Keane, E. F., Kramer, M., Lyne, A. G., Stappers, B. W., \& McLaughlin, M. A. 2011, MNRAS, 415, 3065

Keane, E. F., \& Petroff, E. 2015, MNRAS, 447, 2852

Keane, E. F., Johnston, S., Bhandari, S., et al. 2016, Nature, 530,453

Krishnakumar, M. A., Mitra, D., Naidu, A., Joshi, B. C., \& Manoharan, P. K. 2015, ApJ, 804, 23

Lallement, R., Vergely, J.-L., Valette, B., et al. 2014, A\&A, 561, A91

Lallement, R., Welsh, B. Y., Vergely, J. L., Crifo, F., \& Sfeir, D. 2003, A\&A, 411, 447

Lavalley, M., Isobe, T., \& Feigelson, E. 1992, in Astronomical Society of the Pacific Conference Series, Vol. 25, Astronomical Data Analysis Software and Systems I, ed. D. M. Worrall, C. Biemesderfer, \& J. Barnes, 245

Law, C. J., Backer, D., Yusef-Zadeh, F., \& Maddalena, R. 2009, ApJ, 695, 1070

Law, C. J., Yusef-Zadeh, F., Cotton, W. D., \& Maddalena, R. J. 2008, ApJS, 177, 255

Lawton, B., Gordon, K. D., Babler, B., et al. 2010, ApJ, 716, 453

Lewandowski, W., Rożko, K., Kijak, J., Bhattacharyya, B., \& Roy, J. 2015, MNRAS, 454, 2517

Lorimer, D. R., Bailes, M., McLaughlin, M. A., Narkevic, D. J., \& Crawford, F. 2007, Science, 318, 777

Lorimer, D. R., Faulkner, A. J., Lyne, A. G., et al. 2006, MNRAS, 372, 777

Luan, J., \& Goldreich, P. 2014, ApJ, 785, L26
Lutz, T. E., \& Kelker, D. H. 1973, PASP, 85, 573

Lyne, A. G., Manchester, R. N., \& Taylor, J. H. 1985, MNRAS, 213,613

Macquart, J.-P., \& Koay, J. Y. 2013, ApJ, 776, 125

Madsen, G. J., Reynolds, R. J., \& Haffner, L. M. 2006, ApJ, 652, 401

Manchester, R. N., Fan, G., Lyne, A. G., Kaspi, V. M., \& Crawford, F. 2006, ApJ, 649, 235

Manchester, R. N., \& Taylor, J. H. 1981, AJ, 86, 1953

Masui, K., Lin, H.-H., Sievers, J., et al. 2015, Nature, 528, 523

Matthews, A. M., Nice, D. J., Fonseca, E., et al. 2016, ApJ, 818, 92

McClure-Griffiths, N. M., Dickey, J. M., Gaensler, B. M., \& Green, A. J. 2004, ApJ, 607, L127

McLaughlin, D. E., Anderson, J., Meylan, G., et al. 2006a, ApJS, 166, 249

McLaughlin, M. A., Lyne, A. G., Lorimer, D. R., et al. 2006b, Nature, 439, 817

Nakanishi, H., \& Sofue, Y. 2006, PASJ, 58, 847

Nordgren, T. E., Cordes, J. M., \& Terzian, Y. 1992, AJ, 104, 1465

Oka, T., Onodera, Y., Nagai, M., et al. 2012, ApJS, 201, 14

Oort, J. H., Kerr, F. J., \& Westerhout, G. 1958, MNRAS, 118, 379

Ortolani, S., Barbuy, B., Bica, E., Zoccali, M., \& Renzini, A. 2007, A\&A, 470, 1043

Pennucci, T. T., Possenti, A., Esposito, P., et al. 2015, ApJ, 808, 81

Petroff, E., Bailes, M., Barr, E. D., et al. 2015a, MNRAS, 447, 246

Petroff, E., Johnston, S., Keane, E. F., et al. 2015b, MNRAS, 454, 457

Petroff, E., Barr, E. D., Jameson, A., et al. 2016, PASA, 33, e045

Planck Collaboration, Ade, P. A. R., Aghanim, N., et al. 2014, A\&A, 571, A16

Pshirkov, M. S., Tinyakov, P. G., \& Urban, F. R. 2015, MNRAS, 452, 2851

Purcell, C. R., Gaensler, B. M., Sun, X. H., et al. 2015, ApJ, 804, 22

Puspitarini, L., Lallement, R., Vergely, J.-L., \& Snowden, S. L. 2014, A\&A, 566, A13

Ravi, V., Shannon, R. M., \& Jameson, A. 2015, ApJ, 799, L5

Rea, N., Esposito, P., Pons, J. A., et al. 2013, ApJ, 775, L34

Readhead, A. C. S., \& Duffett-Smith, P. J. 1975, A\&A, 42, 151

Reardon, D. J., Hobbs, G., Coles, W., et al. 2016, MNRAS, 455, 1751

Reis, W., Corradi, W., de Avillez, M. A., \& Santos, F. P. 2011, ApJ, 734, 8

Reynolds, R. J., Scherb, F., \& Roesler, F. L. 1973, ApJ, 185, 869

Rickett, B. J. 1990, Ann. Rev. Astr. Ap., 28, 561

Ridley, J. P., Crawford, F., Lorimer, D. R., et al. 2013, MNRAS, 433, 138

Robin, A. C., Reylé, C., Derrière, S., \& Picaud, S. 2003, A\&A, 409, 523

Savage, B. D., \& Wakker, B. P. 2009, ApJ, 702, 1472

Schnitzeler, D. H. F. M. 2012, MNRAS, 427, 664

Shannon, R. M., \& Johnston, S. 2013, MNRAS, 435, L29

Shklovskii, I. S. 1970, Sov. Astron., 13, 562

Snowden, S. L., Koutroumpa, D., Kuntz, K. D., Lallement, R., \& Puspitarini, L. 2015, ApJ, 806, 120

Snowden, S. L., Freyberg, M. J., Plucinsky, P. P., et al. 1995, ApJ, 454, 643

Spitler, L. G., Cordes, J. M., Hessels, J. W. T., et al. 2014a, ApJ, 790, 101

Spitler, L. G., Lee, K. J., Eatough, R. P., et al. 2014b, ApJ, 780, L3 
Spitler, L. G., Scholz, P., Hessels, J. W. T., et al. 2016, Nature, 531,202

Spitzer, L. 1942, ApJ, 95, 329

Stinebring, D. R. 2006, Chin. J. Atron. Astrophys., Suppl. 2, 6, 204

Storm, J., Carney, B. W., Gieren, W. P., et al. 2004, A\&A, 415, 531

Su, M., Slatyer, T. R., \& Finkbeiner, D. P. 2010, ApJ, 724, 1044

Taylor, J. H., \& Cordes, J. M. 1993, ApJ, 411, 674

Theureau, G., Parent, D., Cognard, I., et al. 2011, A\&A, 525, A94+

Thornton, D., Stappers, B., Bailes, M., et al. 2013, Science, 341, 53

Trang, F. S., \& Rickett, B. J. 2007, ApJ, 661, 1064

Urquhart, J. S., Figura, C. C., Moore, T. J. T., et al. 2014, MNRAS, 437, 1791

van der Marel, R. P., \& Cioni, M.-R. L. 2001, AJ, 122, 1807
Van Eck, C. L., Brown, J. C., Stil, J. M., et al. 2011, ApJ, 728, 97 Vaz, A. I. F., \& Vincente, L. N. 2007, J. Global Optimisation, 39, 197

Verbiest, J. P. W., Weisberg, J. M., Chael, A. A., Lee, K. J., \& Lorimer, D. R. 2012, ApJ, 755, 39

Vivekanand, M., \& Narayan, R. 1982, J. Astrophys. Astr., 3, 399

Walker, A. R. 2012, Ap\&SS, 341, 43

Weisberg, J. M., Boriakoff, V., \& Rankin, J. 1979, A\&A, 77, 204

Welsh, B. Y., Lallement, R., Vergely, J.-L., \& Raimond, S. 2010, A\&A, 510, A54

Welsh, B. Y., Sfeir, D. M., Sirk, M. M., \& Lallememt, R. 1999, A\&A, 352, 308

Williams, P. K. G., \& Berger, E. 2016, Astron. Tel., 8946

Williamson, I. P. 1972, MNRAS, 157, 55+

Wolleben, M. 2007, ApJ, 664, 349

Xu, S., \& Zhang, B. 2016, arXiv:1608.03930 


\section{APPENDIX}

\section{A. DM-INDEPENDENT DISTANCES AND YMW16 MODEL DISTANCES}

In this Appendix we list by category the DM-independent distances used to derive the YMW16 Galactic electron density model. Each table gives the pulsar J2000 name, the Galactic coordinates $(l, b)$, the DM, the distance lower limit $D_{l}$, the estimate distance $D_{i}$ and the distance upper limit $D_{u}$. Detailed references for the source of these data can be obtained from the ATNF Pulsar Catalogue (V1.54). In each table, we also give $D_{m}$, the distance estimate based on the YMW16 Galactic electron density model, and the fractional distance error $D_{\text {err }}$ (expressed as a percentage) defined by Equation 46. Table A6 lists the 27 pulsars with known DM that are believed to be associated with the Magellanic Clouds along with the DM contributions from the Galaxy and the Magellanic Clouds and the model distance. Similarly, Table A7 lists details of the 17 currently known FRBs, the Galactic, Magellanic Cloud and IGM contributions to the DM and the derived FRB source redshifts and co-moving distances. The dispersion measure contribution of the FRB host galaxy, $\mathrm{DM}_{\text {Host }}$, is assumed to be $100 \mathrm{~cm}^{-3} \mathrm{pc}$. All tables list an estimate of the scattering timescale at $1 \mathrm{GHz}$ for each source.

Table A1. Independent distances from measurements of annual parallax

\begin{tabular}{|c|c|c|c|c|c|c|c|c|c|}
\hline $\begin{array}{l}\text { J2000 } \\
\text { Name }\end{array}$ & $\begin{array}{c}l \\
\left({ }^{\circ}\right)\end{array}$ & $\begin{array}{c}b \\
\left({ }^{\circ}\right)\end{array}$ & $\begin{array}{c}\mathrm{DM} \\
\left(\mathrm{cm}^{-3} \mathrm{pc}\right)\end{array}$ & $\begin{array}{c}D_{l} \\
(\mathrm{pc})\end{array}$ & $\begin{array}{c}D_{i} \\
(\mathrm{pc})\end{array}$ & $\begin{array}{c}D_{u} \\
(\mathrm{pc})\end{array}$ & $\begin{array}{l}D_{m} \\
(\mathrm{pc})\end{array}$ & $\begin{array}{c}D_{e r r} \\
(\%)\end{array}$ & $\begin{array}{c}\log \tau_{s c} \\
(\mathrm{~s})\end{array}$ \\
\hline J0023+0923 & 111.383 & -52.849 & 14.30 & 1000 & 4050 & 7100 & 1244 & 0 & -7.700 \\
\hline J0030+0451 & 113.141 & -57.611 & 4.33 & 286 & 303 & 323 & 345 & 7 & -8.971 \\
\hline J0034-0721 & 110.420 & -69.815 & 11.38 & 950 & 1030 & 1110 & 1052 & 0 & -7.966 \\
\hline J0108-1431 & 140.930 & -76.815 & 2.38 & 160 & 210 & 300 & 232 & 0 & -9.554 \\
\hline J0139+5814 & 129.216 & -4.044 & 73.78 & 2400 & 2600 & 2900 & 2006 & 20 & -5.215 \\
\hline J0218+4232 & 139.508 & -17.527 & 61.25 & 2550 & 3150 & 4000 & 2928 & 0 & -5.538 \\
\hline J0332+5434 & 144.995 & -1.221 & 26.76 & 900 & 1000 & 1100 & 1183 & 8 & -6.868 \\
\hline J0358+5413 & 148.190 & 0.811 & 57.14 & 900 & 1000 & 1200 & 1594 & 33 & -5.657 \\
\hline $\mathrm{J} 0437-4715^{\mathrm{a}}$ & 253.394 & -41.963 & 2.64 & 155 & 156 & 157 & 156 & 0 & -9.454 \\
\hline J0452-1759 & 217.078 & -34.087 & 39.90 & 300 & 400 & 600 & 2710 & 352 & -6.254 \\
\hline J0454+5543 & 152.617 & 7.547 & 14.49 & 1130 & 1180 & 1250 & 631 & 79 & -7.684 \\
\hline J0538+2817 & 179.719 & -1.686 & 39.57 & 1100 & 1300 & 1500 & 946 & 16 & -6.267 \\
\hline J0613-0200 & 210.413 & -9.305 & 38.78 & 950 & 1090 & 1270 & 1024 & 0 & -6.299 \\
\hline J0630-2834 & 236.952 & -16.758 & 34.47 & 280 & 320 & 370 & 2072 & 460 & -6.486 \\
\hline J0633+1746 & 195.134 & 4.266 & 2.89 & 170 & 250 & 480 & 138 & 23 & -9.366 \\
\hline J0636+5129 & 163.909 & 18.643 & 11.10 & 182 & 204 & 233 & 209 & 0 & -7.994 \\
\hline J0645+5158 & 163.963 & 20.251 & 18.20 & 625 & 769 & 1000 & 669 & 0 & -7.400 \\
\hline J0659+1414 & 201.108 & 8.258 & 13.98 & 250 & 280 & 310 & 159 & 57 & -7.727 \\
\hline J0737-3039A & 245.236 & -4.505 & 48.92 & 1000 & 1100 & 1300 & 1105 & 0 & -5.919 \\
\hline J0751+1807 & 202.730 & 21.086 & 30.25 & 300 & 400 & 600 & 428 & 0 & -6.686 \\
\hline J0814+7429 & 139.998 & 31.618 & 5.73 & 425 & 432 & 440 & 366 & 16 & -8.692 \\
\hline J0820-1350 & 235.890 & 12.595 & 40.94 & 1800 & 1900 & 2000 & 2321 & 16 & -6.212 \\
\hline J0826+2637 & 196.963 & 31.742 & 19.45 & 270 & 320 & 400 & 313 & 0 & -7.313 \\
\hline J0835-4510 & 263.552 & -2.787 & 67.99 & 260 & 280 & 300 & 328 & 9 & -5.357 \\
\hline J0922+0638 & 225.420 & 36.392 & 27.27 & 1000 & 1100 & 1300 & 1908 & 47 & -6.841 \\
\hline J0953+0755 & 228.908 & 43.697 & 2.96 & 256 & 261 & 266 & 186 & 37 & -9.343 \\
\hline $\mathrm{J} 1012+5307$ & 160.347 & 50.858 & 9.02 & 600 & 700 & 900 & 804 & 0 & -8.222 \\
\hline J1017-7156 & 291.558 & -12.553 & 94.22 & 196 & 256 & 370 & 1807 & 388 & -4.784 \\
\hline $\mathrm{J} 1022+1001$ & 231.795 & 51.101 & 10.25 & 610 & 740 & 930 & 691 & 0 & -8.083 \\
\hline $\mathrm{J} 1023+0038$ & 243.490 & 45.782 & 14.32 & 1328 & 1367 & 1410 & 1057 & 26 & -7.699 \\
\hline J1024-0719 & 251.702 & 40.516 & 6.49 & 800 & 1100 & 1500 & 381 & 110 & -8.566 \\
\hline J1045-4509 & 280.851 & 12.254 & 58.17 & 240 & 340 & 360 & 338 & 0 & -5.626 \\
\hline $\mathrm{J} 1136+1551$ & 241.902 & 69.195 & 4.85 & 330 & 350 & 370 & 415 & 12 & -8.859 \\
\hline $\mathrm{J} 1239+2453$ & 252.450 & 86.541 & 9.24 & 790 & 840 & 900 & 826 & 0 & -8.196 \\
\hline
\end{tabular}


Table A1 (continued)

\begin{tabular}{|c|c|c|c|c|c|c|c|c|c|}
\hline $\begin{array}{l}\text { J2000 } \\
\text { Name }\end{array}$ & $\begin{array}{c}l \\
\left(^{\circ}\right)\end{array}$ & $\begin{array}{c}b \\
\left(^{\circ}\right)\end{array}$ & $\begin{array}{c}\mathrm{DM} \\
\left(\mathrm{cm}^{-3} \mathrm{pc}\right)\end{array}$ & $\begin{array}{c}D_{l} \\
(\mathrm{pc})\end{array}$ & $\begin{array}{c}D_{i} \\
(\mathrm{pc})\end{array}$ & $\begin{array}{c}D_{u} \\
(\mathrm{pc})\end{array}$ & $\begin{array}{l}D_{m} \\
(\mathrm{pc})\end{array}$ & $\begin{array}{c}D_{\text {err }} \\
(\%)\end{array}$ & $\begin{array}{c}\log \tau_{s c} \\
(\mathrm{~s})\end{array}$ \\
\hline $\mathrm{J} 1300+1240$ & 311.310 & 75.414 & 10.17 & 500 & 600 & 800 & 878 & 10 & -8.092 \\
\hline J1456-6843 & 313.869 & -8.543 & 8.60 & 380 & 430 & 490 & 436 & 0 & -8.273 \\
\hline $\mathrm{J} 1509+5531$ & 91.325 & 52.287 & 19.61 & 2000 & 2100 & 2200 & 2072 & 0 & -7.302 \\
\hline $\mathrm{J} 1537+1155^{\mathrm{a}}$ & 19.848 & 48.341 & 11.61 & 961 & 1162 & 1471 & 876 & 10 & -7.944 \\
\hline $\mathrm{J} 1543+0929$ & 17.811 & 45.775 & 35.24 & 5400 & 5900 & 6500 & 25000 & 285 & -6.451 \\
\hline J1559-4438 & 334.540 & 6.367 & 56.10 & 2000 & 2300 & 2800 & 1480 & 35 & -5.688 \\
\hline J1600-3053 & 344.090 & 16.451 & 52.33 & 1500 & 1800 & 2300 & 2535 & 10 & -5.806 \\
\hline J1603-7202 & 316.630 & -14.496 & 38.05 & 370 & 530 & 570 & 1129 & 98 & -6.330 \\
\hline J1614-2230 & 352.636 & 20.192 & 34.50 & 400 & 700 & 1000 & 1395 & 40 & -6.484 \\
\hline $\mathrm{J} 1640+2224$ & 41.051 & 38.271 & 18.42 & 1450 & 2425 & 3400 & 1502 & 0 & -7.384 \\
\hline J1643-1224 & 5.669 & 21.218 & 62.41 & 640 & 740 & 860 & 791 & 0 & -5.505 \\
\hline $\mathrm{J} 1713+0747$ & 28.751 & 25.223 & 15.99 & 1136 & 1176 & 1220 & 919 & 24 & -7.564 \\
\hline J1730-2304 & 3.137 & 6.023 & 9.62 & 520 & 620 & 770 & 512 & 2 & -8.153 \\
\hline $\mathrm{J} 1738+0333$ & 27.721 & 17.742 & 33.77 & 1370 & 1470 & 1587 & 1505 & 0 & -6.518 \\
\hline $\mathrm{J} 1741+1351$ & 37.885 & 21.641 & 24.21 & 1020 & 1075 & 1136 & 1363 & 20 & -7.013 \\
\hline J1744-1134 & 14.794 & 9.180 & 3.14 & 384 & 395 & 406 & 148 & 159 & -9.286 \\
\hline J1756-2251 & 6.499 & 0.948 & 121.18 & 490 & 730 & 1330 & 2806 & 111 & -4.334 \\
\hline J1900-2600 & 10.342 & -13.451 & 37.99 & 500 & 700 & 1100 & 1237 & 12 & -6.332 \\
\hline J1909-3744 & 359.731 & -19.596 & 10.39 & 1230 & 1234 & 1239 & 564 & 118 & -8.068 \\
\hline J1918-0642 & 30.027 & -9.123 & 26.55 & 769 & 909 & 1111 & 1026 & 0 & -6.880 \\
\hline $\mathrm{J} 1932+1059$ & 47.382 & -3.884 & 3.18 & 250 & 310 & 400 & 229 & 9 & -9.273 \\
\hline J1935+1616 & 52.436 & -2.093 & 158.52 & 2900 & 3700 & 5000 & 4314 & 0 & -3.850 \\
\hline J1939+2134 & 57.509 & -0.290 & 71.04 & 1200 & 1500 & 2000 & 2897 & 45 & -5.281 \\
\hline $\mathrm{J} 1944+0907$ & 47.160 & -7.357 & 24.34 & 670 & 1335 & 2000 & 1218 & 0 & -7.005 \\
\hline $\mathrm{J} 2017+0603$ & 48.621 & -16.026 & 23.92 & 1250 & 1560 & 1870 & 1398 & 0 & -7.030 \\
\hline J2018+2839 & 68.099 & -3.983 & 14.17 & 890 & 980 & 1090 & 957 & 0 & -7.711 \\
\hline $\mathrm{J} 2022+2854$ & 68.863 & -4.671 & 24.64 & 700 & 2100 & 2700 & 1707 & 0 & -6.988 \\
\hline $\mathrm{J} 2022+5154$ & 87.862 & 8.380 & 22.65 & 1600 & 1800 & 2100 & 1424 & 12 & -7.106 \\
\hline $\mathrm{J} 2043+1711$ & 61.919 & -15.313 & 20.71 & 1000 & 1250 & 1667 & 1473 & 0 & -7.229 \\
\hline J2048-1616 & 30.514 & -33.077 & 11.46 & 920 & 950 & 970 & 775 & 19 & -7.958 \\
\hline $\mathrm{J} 2055+3630$ & 79.133 & -5.589 & 97.31 & 4400 & 5000 & 5800 & 5240 & 0 & -4.726 \\
\hline J2124-3358 & 10.925 & -45.438 & 4.60 & 360 & 410 & 500 & 360 & 0 & -8.912 \\
\hline $\mathrm{J} 2129-5721^{\mathrm{a}}$ & 338.005 & -43.570 & 31.85 & 1700 & 3200 & 4700 & 6170 & 31 & -6.608 \\
\hline J2144-3933 & 2.794 & -49.466 & 3.35 & 150 & 160 & 180 & 289 & 61 & -9.223 \\
\hline $\mathrm{J} 2145-0750$ & 47.777 & -42.084 & 9.00 & 480 & 530 & 590 & 693 & 18 & -8.224 \\
\hline $\mathrm{J} 2157+4017$ & 90.488 & -11.341 & 70.86 & 2500 & 2900 & 3400 & 4750 & 40 & -5.285 \\
\hline $\mathrm{J} 2214+3000$ & 86.855 & -21.665 & 22.56 & 909 & 1000 & 1111 & 1674 & 51 & -7.112 \\
\hline $\mathrm{J} 2222-0137$ & 62.018 & -46.075 & 3.28 & 266 & 267 & 268 & 267 & 0 & -9.243 \\
\hline $\mathrm{J} 2313+4253$ & 104.410 & -16.422 & 17.28 & 1000 & 1060 & 1160 & 1109 & 0 & -7.466 \\
\hline
\end{tabular}

${ }^{a}$ Distance estimate based on measured $\dot{P}_{b}$

Table A2. Kinematic distances from HI absorption measurements

\begin{tabular}{cccccccccc}
\hline \hline $\begin{array}{c}\mathrm{J} 2000 \\
\text { Name }\end{array}$ & $\begin{array}{c}l \\
\left({ }^{\circ}\right)\end{array}$ & $\begin{array}{c}b \\
\left({ }^{\circ}\right)\end{array}$ & $\begin{array}{c}\mathrm{DM} \\
\left(\mathrm{cm}^{-3} \mathrm{pc}\right)\end{array}$ & $\begin{array}{c}D_{l} \\
(\mathrm{pc})\end{array}$ & $\begin{array}{c}D_{i} \\
(\mathrm{pc})\end{array}$ & $\begin{array}{c}D_{u} \\
(\mathrm{pc})\end{array}$ & $\begin{array}{c}D_{m} \\
(\mathrm{pc})\end{array}$ & $\begin{array}{c}D_{\text {err }} \\
(\%)\end{array}$ & $\begin{array}{c}\log \tau_{s c} \\
(\mathrm{~s})\end{array}$ \\
\hline $\mathrm{J} 0141+6009$ & 129.147 & -2.105 & 34.80 & 1600 & 2300 & 3000 & 1495 & 7 & -6.471 \\
\hline
\end{tabular}


Table A2 (continued)

\begin{tabular}{|c|c|c|c|c|c|c|c|c|c|}
\hline $\begin{array}{l}\text { J2000 } \\
\text { Name }\end{array}$ & $\begin{array}{c}l \\
\left(^{\circ}\right)\end{array}$ & $\begin{array}{c}b \\
\left({ }^{\circ}\right)\end{array}$ & $\begin{array}{c}\mathrm{DM} \\
\left(\mathrm{cm}^{-3} \mathrm{pc}\right)\end{array}$ & $\begin{array}{c}D_{l} \\
(\mathrm{pc})\end{array}$ & $\begin{array}{c}D_{i} \\
(\mathrm{pc})\end{array}$ & $\begin{array}{l}D_{u} \\
(\mathrm{pc})\end{array}$ & $\begin{array}{l}D_{m} \\
(\mathrm{pc})\end{array}$ & $\begin{array}{c}D_{e r r} \\
(\%)\end{array}$ & $\begin{array}{c}\log \tau_{s c} \\
\quad(\mathrm{~s})\end{array}$ \\
\hline J0738-4042 & 254.194 & -9.192 & 160.80 & 800 & 1600 & 2400 & 1563 & 0 & -3.824 \\
\hline J0742-2822 & 243.773 & -2.444 & 73.78 & 1200 & 2000 & 3000 & 3115 & 4 & -5.215 \\
\hline J0837-4135 & 260.904 & -0.336 & 147.29 & 600 & 1500 & 2700 & 1426 & 0 & -3.983 \\
\hline J0908-4913 & 270.266 & -1.019 & 180.37 & 300 & 1000 & 1700 & 1023 & 0 & -3.617 \\
\hline J0942-5552 & 278.571 & -2.230 & 180.20 & 100 & 300 & 1100 & 415 & 0 & -3.618 \\
\hline $\mathrm{J} 1001-5507$ & 280.226 & 0.085 & 130.32 & 0 & 300 & 1400 & 408 & 0 & -4.203 \\
\hline $\mathrm{J} 1048-5832$ & 287.425 & 0.577 & 129.10 & 2200 & 2900 & 4100 & 1793 & 23 & -4.220 \\
\hline $\mathrm{J} 1056-6258$ & 290.292 & -2.966 & 320.30 & 1900 & 2400 & 2900 & 2613 & 0 & -2.574 \\
\hline $\mathrm{J} 1141-6545$ & 295.791 & -3.863 & 116.08 & 1000 & 3000 & 5000 & 1676 & 0 & -4.411 \\
\hline $\mathrm{J} 1157-6224$ & 296.705 & -0.199 & 325.20 & 2000 & 4000 & 6000 & 4758 & 0 & -2.546 \\
\hline $\mathrm{J} 1224-6407$ & 299.984 & -1.415 & 97.47 & 2000 & 4000 & 6000 & 1534 & 30 & -4.723 \\
\hline $\mathrm{J} 1243-6423$ & 302.051 & -1.532 & 297.25 & 0 & 2000 & 4000 & 9411 & 135 & -2.710 \\
\hline $\mathrm{J} 1326-5859$ & 307.504 & 3.565 & 287.30 & 2000 & 3000 & 5000 & 10688 & 114 & -2.772 \\
\hline $\mathrm{J} 1327-6222$ & 307.074 & 0.204 & 318.80 & 2000 & 4000 & 6000 & 6514 & 9 & -2.582 \\
\hline $\mathrm{J} 1359-6038$ & 311.239 & 1.126 & 293.71 & 3000 & 5000 & 7000 & 5472 & 0 & -2.732 \\
\hline $\mathrm{J} 1401-6357$ & 310.568 & -2.140 & 98.00 & 1200 & 1800 & 2500 & 1762 & 0 & -4.714 \\
\hline $\mathrm{J} 1453-6413$ & 315.733 & -4.427 & 71.07 & 2000 & 2800 & 4100 & 1432 & 40 & -5.280 \\
\hline $\mathrm{J} 1600-5044$ & 330.690 & 1.631 & 260.56 & 6000 & 6900 & 8800 & 5110 & 17 & -2.949 \\
\hline $\mathrm{J} 1602-5100$ & 330.688 & 1.286 & 170.93 & 7300 & 8000 & 8900 & 3407 & 114 & -3.714 \\
\hline J1644-4559 & 339.193 & -0.195 & 478.80 & 4100 & 4500 & 4900 & 4437 & 0 & -1.842 \\
\hline $\mathrm{J} 1651-4246$ & 342.457 & 0.923 & 482.00 & 4600 & 5200 & 7300 & 5753 & 0 & -1.830 \\
\hline $\mathrm{J} 1707-4053$ & 345.718 & -0.198 & 360.00 & 3000 & 4000 & 6000 & 3912 & 0 & -2.361 \\
\hline $\mathrm{J} 1709-4429$ & 343.098 & -2.686 & 75.69 & 2000 & 2600 & 3100 & 2275 & 0 & -5.170 \\
\hline $\mathrm{J} 1721-3532$ & 351.687 & 0.670 & 496.00 & 4000 & 4600 & 5200 & 4705 & 0 & -1.777 \\
\hline $\mathrm{J} 1740-3015$ & 358.294 & 0.238 & 152.15 & 100 & 400 & 2100 & 2945 & 40 & -3.924 \\
\hline $\mathrm{J} 1741-2054^{\mathrm{a}}$ & 6.425 & 4.909 & 4.70 & 300 & 650 & 1000 & 273 & 10 & -8.890 \\
\hline $\mathrm{J} 1745-3040$ & 358.553 & -0.963 & 88.37 & 0 & 200 & 1300 & 2343 & 80 & -4.897 \\
\hline $\mathrm{J} 1752-2806$ & 1.540 & -0.961 & 50.37 & 100 & 200 & 1300 & 1335 & 3 & -5.870 \\
\hline $\mathrm{J} 1801-2304$ & 6.837 & -0.066 & 1073.90 & 3000 & 4000 & 5000 & 6522 & 30 & -0.369 \\
\hline $\mathrm{J} 1807-0847$ & 20.061 & 5.587 & 112.38 & 600 & 1500 & 2700 & 2700 & 0 & -4.469 \\
\hline J1809-1943 & 10.727 & -0.158 & 178.00 & 3100 & 3600 & 4100 & 3153 & 0 & -3.641 \\
\hline $\mathrm{J} 1820-0427$ & 25.456 & 4.733 & 84.44 & 100 & 300 & 900 & 2918 & 224 & -4.978 \\
\hline $\mathrm{J} 1823+0550$ & 34.987 & 8.859 & 66.78 & 1200 & 2000 & 3300 & 3089 & 0 & -5.388 \\
\hline J1824-1945 & 12.279 & -3.106 & 224.65 & 2800 & 3700 & 4300 & 5612 & 31 & -3.219 \\
\hline $\mathrm{J} 1825-0935$ & 21.449 & 1.324 & 19.38 & 100 & 300 & 1000 & 261 & 0 & -7.317 \\
\hline $\mathrm{J} 1832-0827$ & 23.272 & 0.298 & 300.87 & 4800 & 5200 & 5700 & 4046 & 19 & -2.688 \\
\hline $\mathrm{J} 1833-0827$ & 23.386 & 0.063 & 411.00 & 4000 & 4500 & 5000 & 4381 & 0 & -2.120 \\
\hline $\mathrm{J} 1848-0123$ & 31.339 & 0.039 & 159.53 & 4000 & 4400 & 4800 & 3532 & 13 & -3.839 \\
\hline $\mathrm{J} 1852+0031$ & 33.523 & 0.017 & 787.00 & 6000 & 8000 & 10000 & 6400 & 0 & -0.936 \\
\hline $\mathrm{J} 1857+0212$ & 35.617 & -0.390 & 506.77 & 6000 & 8000 & 10000 & 5932 & 1 & -1.738 \\
\hline $\mathrm{J} 1901+0331$ & 37.213 & -0.637 & 402.08 & 5000 & 7000 & 9000 & 6053 & 0 & -2.160 \\
\hline $\mathrm{J} 1901+0716$ & 40.569 & 1.056 & 252.81 & 2700 & 3400 & 4300 & 7237 & 68 & -3.004 \\
\hline $\mathrm{J} 1902+0556$ & 39.501 & 0.210 & 177.49 & 3100 & 3600 & 4200 & 4198 & 0 & -3.646 \\
\hline $\mathrm{J} 1902+0615$ & 39.814 & 0.336 & 502.90 & 5000 & 7000 & 10000 & 7174 & 0 & -1.752 \\
\hline $\mathrm{J} 1903+0135$ & 35.727 & -1.955 & 245.17 & 2800 & 3300 & 3900 & 6000 & 54 & -3.060 \\
\hline $\mathrm{J} 1906+0641$ & 40.604 & -0.304 & 472.80 & 5000 & 7000 & 9000 & 6816 & 0 & -1.865 \\
\hline $\mathrm{J} 1906+0746$ & 41.598 & 0.147 & 217.75 & 6000 & 7400 & 9900 & 4814 & 25 & -3.275 \\
\hline $\mathrm{J} 1909+0254$ & 37.605 & -2.713 & 171.73 & 3600 & 4500 & 7700 & 5883 & 0 & -3.706 \\
\hline $\mathrm{J} 1909+1102$ & 44.832 & 0.992 & 149.98 & 4000 & 4800 & 5900 & 4788 & 0 & -3.950 \\
\hline $\mathrm{J} 1915+1009$ & 44.707 & -0.651 & 241.69 & 5000 & 7000 & 9000 & 5990 & 0 & -3.086 \\
\hline
\end{tabular}


Table A2 (continued)

\begin{tabular}{|c|c|c|c|c|c|c|c|c|c|}
\hline $\begin{array}{l}\text { J2000 } \\
\text { Name }\end{array}$ & $\begin{array}{c}l \\
\left(^{\circ}\right)\end{array}$ & $\begin{array}{c}b \\
\left(^{\circ}\right)\end{array}$ & $\begin{array}{c}\mathrm{DM} \\
\left(\mathrm{cm}^{-3} \mathrm{pc}\right)\end{array}$ & $\begin{array}{c}D_{l} \\
(\mathrm{pc})\end{array}$ & $\begin{array}{c}D_{i} \\
(\mathrm{pc})\end{array}$ & $\begin{array}{l}D_{u} \\
(\mathrm{pc})\end{array}$ & $\begin{array}{l}D_{m} \\
(\mathrm{pc})\end{array}$ & $\begin{array}{c}D_{e r r} \\
(\%)\end{array}$ & $\begin{array}{c}\log \tau_{s c} \\
\text { (s) }\end{array}$ \\
\hline $\mathrm{J} 1916+1312$ & 47.576 & 0.451 & 237.01 & 3600 & 4500 & 5700 & 6351 & 11 & -3.121 \\
\hline $\mathrm{J} 1917+1353$ & 48.260 & 0.624 & 94.54 & 4000 & 5000 & 6000 & 2940 & 36 & -4.777 \\
\hline $\mathrm{J} 1921+2153$ & 55.777 & 3.501 & 12.44 & 100 & 300 & 1100 & 809 & 0 & -7.865 \\
\hline $\mathrm{J} 1922+2110$ & 55.278 & 2.935 & 217.09 & 2000 & 4000 & 6000 & 7041 & 17 & -3.281 \\
\hline $\mathrm{J} 1926+1648$ & 51.859 & 0.063 & 176.88 & 4000 & 6000 & 9000 & 4495 & 0 & -3.652 \\
\hline $\mathrm{J} 1932+2020$ & 55.575 & 0.639 & 211.15 & 3000 & 5000 & 8000 & 4964 & 0 & -3.331 \\
\hline $\mathrm{J} 1932+2220$ & 57.356 & 1.554 & 219.20 & 10100 & 10900 & 12200 & 7999 & 26 & -3.263 \\
\hline $\mathrm{J} 1946+1805$ & 55.326 & -3.500 & 16.22 & 100 & 300 & 900 & 1010 & 12 & -7.546 \\
\hline J2004+3137 & 69.011 & 0.021 & 234.82 & 7000 & 8000 & 10000 & 7264 & 0 & -3.138 \\
\hline $\mathrm{J} 2021+3651^{\mathrm{a}}$ & 75.222 & 0.111 & 367.50 & 400 & 1800 & 3500 & 10512 & 200 & -2.324 \\
\hline $\mathrm{J} 2113+4644$ & 89.003 & -1.266 & 141.26 & 3000 & 4000 & 5000 & 4123 & 0 & -4.058 \\
\hline $\mathrm{J} 2257+5909$ & 108.831 & -0.575 & 151.08 & 2000 & 3000 & 4000 & 3151 & 0 & -3.937 \\
\hline $\mathrm{J} 2321+6024$ & 112.095 & -0.566 & 94.59 & 1800 & 2700 & 3900 & 2513 & 0 & -4.777 \\
\hline
\end{tabular}

${ }^{a}$ Distance estimate based on $\mathrm{X}$-ray absorption

Table A3. Independent distances from globular cluster associations

\begin{tabular}{|c|c|c|c|c|c|c|c|c|c|c|}
\hline $\begin{array}{l}\text { J2000 } \\
\text { Name }\end{array}$ & $\begin{array}{l}\text { Globular } \\
\text { Cluster }\end{array}$ & $\begin{array}{c}l \\
\left(^{\circ}\right)\end{array}$ & $\begin{array}{c}b \\
\left(^{\circ}\right)\end{array}$ & $\begin{array}{c}\mathrm{DM} \\
\left(\mathrm{cm}^{-3} \mathrm{pc}\right)\end{array}$ & $\begin{array}{c}D_{l} \\
(\mathrm{pc})\end{array}$ & $\begin{array}{c}D_{i} \\
(\mathrm{pc})\end{array}$ & $\begin{array}{l}D_{u} \\
(\mathrm{pc})\end{array}$ & $\begin{array}{l}D_{m} \\
(\mathrm{pc})\end{array}$ & $\begin{array}{c}D_{\text {err }} \\
(\%)\end{array}$ & $\begin{array}{c}\log \tau_{s c} \\
(\mathrm{~s})\end{array}$ \\
\hline J0024-7204C & 47Tuc & 305.923 & -44.892 & 24.40 & 3650 & 4000 & 4350 & 2547 & 43 & -7.002 \\
\hline J0514-4002A & NGC1851 & 244.514 & -35.036 & 52.15 & 12100 & 12650 & 13200 & 25000 & 89 & -5.812 \\
\hline $\mathrm{J} 1312+1810$ & M53 & 332.954 & 79.763 & 24.00 & 17200 & 18900 & 20600 & 25000 & 21 & -7.025 \\
\hline $\mathrm{J} 1342+2822 \mathrm{~A}$ & M3 & 42.209 & 78.709 & 26.50 & 9600 & 9900 & 10200 & 25000 & 145 & -6.883 \\
\hline $\mathrm{J} 1518+0204 \mathrm{~A}$ & M5 & 3.870 & 46.802 & 29.40 & 7000 & 7500 & 8000 & 7533 & 0 & -6.729 \\
\hline J1623-2631 & M4 & 350.976 & 15.960 & 62.86 & 1600 & 1800 & 2000 & 3651 & 83 & -5.493 \\
\hline $\mathrm{J} 1641+3627 \mathrm{~A}$ & M13 & 59.000 & 40.914 & 30.60 & 7200 & 7700 & 8200 & 7760 & 0 & -6.669 \\
\hline $\mathrm{J} 1701-3006 \mathrm{~A}$ & M62 & 353.578 & 7.322 & 114.97 & 6470 & 7050 & 7630 & 4881 & 33 & -4.428 \\
\hline J1721-1936 & NGC6342 & 4.857 & 9.738 & 75.70 & 7800 & 8400 & 9000 & 3070 & 154 & -5.170 \\
\hline $\mathrm{J} 1740-5340 \mathrm{~A}$ & NGC6397 & 338.165 & -11.967 & 71.80 & 1500 & 2200 & 2700 & 3140 & 16 & -5.262 \\
\hline $\mathrm{J} 1748-2021 \mathrm{~A}$ & NGC6440 & 7.728 & 3.801 & 219.40 & 7600 & 8200 & 8800 & 8551 & 0 & -3.262 \\
\hline $\mathrm{J} 1748-2446 \mathrm{~A}$ & Ter5 & 3.836 & 1.696 & 239.00 & 4600 & 5500 & 6400 & 4410 & 4 & -3.106 \\
\hline $\mathrm{J} 1750-3703 \mathrm{~A}$ & NGC6441 & 353.532 & -5.009 & 233.82 & 12500 & 13500 & 14500 & 13527 & 0 & -3.146 \\
\hline $\mathrm{J} 1801-0857 \mathrm{~A}$ & NGC6517 & 19.225 & 6.762 & 182.56 & 6500 & 7200 & 7900 & 6679 & 0 & -3.595 \\
\hline $\mathrm{J} 1803-30$ & NGC6522 & 1.025 & -3.926 & 192.00 & 6240 & 7800 & 9360 & 5749 & 9 & -3.504 \\
\hline J1804-0735 & NGC6539 & 20.792 & 6.773 & 186.32 & 7800 & 8400 & 9000 & 8156 & 0 & -3.558 \\
\hline $\mathrm{J} 1807-2459 \mathrm{~A}$ & NGC6544 & 5.837 & -2.203 & 134.00 & 2540 & 2790 & 3040 & 3015 & 0 & -4.153 \\
\hline $\mathrm{J} 1823-3021 \mathrm{~A}$ & NGC6624 & 2.788 & -7.913 & 86.88 & 7800 & 8400 & 9000 & 3145 & 148 & -4.927 \\
\hline $\mathrm{J} 1824-2452 \mathrm{~A}$ & M28 & 7.797 & -5.578 & 120.50 & 5200 & 5500 & 5800 & 3737 & 39 & -4.344 \\
\hline J1835-3259A & NGC6652 & 1.532 & -11.371 & 63.35 & 10200 & 10700 & 11200 & 2711 & 276 & -5.480 \\
\hline $\mathrm{J} 1836-2354 \mathrm{~A}$ & M22 & 9.886 & -7.561 & 89.11 & 2900 & 3200 & 3500 & 3269 & 0 & -4.882 \\
\hline $\mathrm{J} 1905+0154 \mathrm{~A}$ & NGC6749 & 36.208 & -2.201 & 193.69 & 13950 & 14450 & 14950 & 5509 & 153 & -3.488 \\
\hline J1910-5959A & NGC6752 & 336.525 & -25.730 & 33.28 & 4490 & 4550 & 4610 & 1642 & 173 & -6.540 \\
\hline $\mathrm{J} 1911+0101 \mathrm{~A}$ & NGC6760 & 36.111 & -3.918 & 199.00 & 8700 & 9500 & 10300 & 8869 & 0 & -3.439 \\
\hline $\mathrm{J} 1953+1846 \mathrm{~A}$ & M71 & 56.744 & -4.563 & 117.00 & 6000 & 6450 & 6900 & 4505 & 33 & -4.397 \\
\hline $\mathrm{J} 2129+1210 \mathrm{~A}$ & M15 & 65.012 & -27.312 & 67.00 & 12900 & 13550 & 14200 & 25000 & 76 & -5.383 \\
\hline $\mathrm{J} 2140-2310 \mathrm{~A}$ & M30 & 27.179 & -46.837 & 25.06 & 9200 & 9450 & 9700 & 3112 & 196 & -6.963 \\
\hline
\end{tabular}


Table A4 (continued)

\begin{tabular}{|c|c|c|c|c|c|c|c|c|c|c|}
\hline $\begin{array}{l}\text { J2000 } \\
\text { Name }\end{array}$ & Nebula & $\begin{array}{c}l \\
\left({ }^{\circ}\right)\end{array}$ & $\begin{array}{c}b \\
\left({ }^{\circ}\right)\end{array}$ & $\begin{array}{c}\mathrm{DM} \\
\left(\mathrm{cm}^{-3} \mathrm{pc}\right)\end{array}$ & $\begin{array}{c}D_{l} \\
(\mathrm{pc})\end{array}$ & $\begin{array}{c}D_{i} \\
(\mathrm{pc})\end{array}$ & $\begin{array}{c}D_{u} \\
(\mathrm{pc})\end{array}$ & $\begin{array}{l}D_{m} \\
(\mathrm{pc})\end{array}$ & $\begin{array}{c}D_{\text {err }} \\
(\%)\end{array}$ & $\begin{array}{c}\log \tau_{s c} \\
(\mathrm{~s})\end{array}$ \\
\hline
\end{tabular}

Table A4. Independent distances from nebular associations

\begin{tabular}{|c|c|c|c|c|c|c|c|c|c|c|}
\hline $\begin{array}{l}\text { J2000 } \\
\text { Name }\end{array}$ & Nebula & $\begin{array}{c}l \\
\left({ }^{\circ}\right)\end{array}$ & $\begin{array}{c}b \\
\left({ }^{\circ}\right)\end{array}$ & $\begin{array}{c}\mathrm{DM} \\
\left(\mathrm{cm}^{-3} \mathrm{pc}\right)\end{array}$ & $\begin{array}{c}D_{l} \\
(\mathrm{pc})\end{array}$ & $\begin{array}{c}D_{i} \\
(\mathrm{pc})\end{array}$ & $\begin{array}{c}D_{u} \\
(\mathrm{pc})\end{array}$ & $\begin{array}{l}D_{m} \\
(\mathrm{pc})\end{array}$ & $\begin{array}{c}D_{\text {err }} \\
(\%)\end{array}$ & $\begin{array}{c}\log \tau_{s c} \\
(\mathrm{~s})\end{array}$ \\
\hline J0248+6021 & HII:W5 & 136.903 & 0.697 & 370.00 & 1800 & 2000 & 2200 & 25000 & 1036 & -2.311 \\
\hline J0534+2200 & PWN:Crab & 184.558 & -5.784 & 56.79 & 1500 & 2000 & 2500 & 1311 & 14 & -5.667 \\
\hline J1124-5916 & SNR:G292.0+1.8 & 292.038 & 1.752 & 330.00 & 3000 & 5000 & 8000 & 2678 & 12 & -2.520 \\
\hline J1400-6325 & SNR:G310.6-1.6 & 310.592 & -1.593 & 563.00 & 5000 & 7000 & 9000 & 9169 & 2 & -1.547 \\
\hline $\mathrm{J} 1513-5908$ & SNR:G320.4-1.2 & 320.321 & -1.162 & 252.50 & 3600 & 4400 & 5700 & 4450 & 0 & -3.006 \\
\hline J1550-5418 & SNR:G327.24-0.1 & 327.237 & -0.132 & 830.00 & 3500 & 4000 & 4500 & 6291 & 40 & -0.839 \\
\hline $\mathrm{J} 1856+0113$ & SNR:W44 & 34.560 & -0.497 & 96.74 & 2700 & 3300 & 3900 & 2820 & 0 & -4.737 \\
\hline $\mathrm{J} 1930+1852$ & SNR:G54.1+0.3 & 54.096 & 0.265 & 308.00 & 5000 & 7000 & 10000 & 6191 & 0 & -2.645 \\
\hline $\mathrm{J} 1952+3252$ & SNR:CTB80 & 68.765 & 2.823 & 45.01 & 1000 & 3000 & 5000 & 3221 & 0 & -6.057 \\
\hline $\mathrm{J} 2229+6114$ & SNR:G106.6+2.9 & 106.647 & 2.949 & 204.97 & 2400 & 3000 & 3600 & 5037 & 40 & -3.385 \\
\hline $\mathrm{J} 2337+6151$ & SNR:G114.3+0.3 & 114.284 & 0.233 & 58.41 & 600 & 700 & 800 & 2079 & 160 & -5.619 \\
\hline
\end{tabular}

Table A5. Independent distances from stellar companions

\begin{tabular}{|c|c|c|c|c|c|c|c|c|c|c|}
\hline $\begin{array}{l}\text { J2000 } \\
\text { Name }\end{array}$ & $\begin{array}{l}\text { Star } \\
\text { Type }\end{array}$ & $\begin{array}{c}l \\
\left({ }^{\circ}\right)\end{array}$ & $\begin{array}{c}b \\
\left({ }^{\circ}\right)\end{array}$ & $\begin{array}{c}\mathrm{DM} \\
\left(\mathrm{cm}^{-3} \mathrm{pc}\right)\end{array}$ & $\begin{array}{c}D_{l} \\
(\mathrm{pc})\end{array}$ & $\begin{array}{c}D_{i} \\
(\mathrm{pc})\end{array}$ & $\begin{array}{c}D_{u} \\
(\mathrm{pc})\end{array}$ & $\begin{array}{l}D_{m} \\
(\mathrm{pc})\end{array}$ & $\begin{array}{c}D_{e r r} \\
(\%)\end{array}$ & $\begin{array}{c}\log \tau_{s c} \\
(\mathrm{~s})\end{array}$ \\
\hline $\mathrm{J} 0337+1715$ & WD & 169.990 & -30.039 & 21.32 & 1220 & 1300 & 1380 & 817 & 49 & -7.189 \\
\hline $\mathrm{J} 0348+0432$ & WD & 183.337 & -36.774 & 40.46 & 1900 & 2100 & 2300 & 2255 & 0 & -6.231 \\
\hline J0614-3329 & WD & 240.501 & -21.827 & 37.05 & 760 & 890 & 1020 & 2691 & 164 & -6.372 \\
\hline $\mathrm{J} 1227-4853$ & MS-G & 298.965 & 13.796 & 43.42 & 1800 & 1900 & 2000 & 1244 & 45 & -6.116 \\
\hline $\mathrm{J} 1231-1411$ & WD & 295.531 & 48.385 & 8.09 & 350 & 430 & 510 & 420 & 0 & -8.338 \\
\hline $\mathrm{J} 1302-6350$ & $\mathrm{Be}$ & 304.184 & -0.992 & 146.72 & 1900 & 2300 & 2700 & 2213 & 0 & -3.990 \\
\hline $\mathrm{J} 1544+4937$ & $\mathrm{UL}$ & 79.172 & 50.166 & 23.22 & 2000 & 3500 & 5000 & 2988 & 0 & -7.071 \\
\hline $\mathrm{J} 1903+0327$ & MS & 37.336 & -1.014 & 297.52 & 6000 & 7000 & 8000 & 6122 & 0 & -2.708 \\
\hline $\mathrm{J} 2032+4127$ & $\mathrm{Be}$ & 80.224 & 1.028 & 114.65 & 1400 & 1500 & 1700 & 4623 & 172 & -4.433 \\
\hline
\end{tabular}


Table A6. Pulsars in the Magellanic Clouds

\begin{tabular}{|c|c|c|c|c|c|c|c|c|c|c|c|}
\hline $\begin{array}{l}\text { J2000 } \\
\text { Name }\end{array}$ & Cloud & $\begin{array}{c}l \\
\left(^{\circ}\right)\end{array}$ & $\begin{array}{c}b \\
\left({ }^{\circ}\right)\end{array}$ & $\begin{array}{c}\mathrm{DM} \\
\left(\mathrm{cm}^{-3} \mathrm{pc}\right)\end{array}$ & $\begin{array}{c}D_{l} \\
(\mathrm{pc})\end{array}$ & $\begin{array}{c}D_{i} \\
(\mathrm{pc})\end{array}$ & $\begin{array}{l}D_{u} \\
(\mathrm{pc})\end{array}$ & $\begin{array}{c}\mathrm{DM}_{\mathrm{Gal}} \\
\left(\mathrm{cm}^{-3} \mathrm{pc}\right)\end{array}$ & $\begin{array}{c}\mathrm{DM}_{\mathrm{MC}} \\
\left(\mathrm{cm}^{-3} \mathrm{pc}\right)\end{array}$ & $\begin{array}{l}D_{m} \\
(\mathrm{pc})\end{array}$ & $\begin{array}{c}\log \tau_{s c} \\
(\mathrm{~s})\end{array}$ \\
\hline J0045-7042 & $\mathrm{SMC}$ & 303.652 & -46.418 & 70 & 54000 & 59700 & 66000 & 28.87 & 41.13 & 58520 & -6.505 \\
\hline J0045-7319 & $\mathrm{SMC}$ & 303.514 & -43.804 & 105.4 & 54000 & 59700 & 66000 & 30.70 & 74.70 & 58725 & -5.494 \\
\hline J0111-7131 & $\mathrm{SMC}$ & 300.669 & -45.510 & 76 & 54000 & 59700 & 66000 & 29.31 & 46.69 & 59164 & -6.298 \\
\hline J0113-7220 & $\mathrm{SMC}$ & 300.615 & -44.688 & 125.49 & 54000 & 59700 & 66000 & 30.02 & 95.47 & 60998 & -5.061 \\
\hline J0131-7310 & $\mathrm{SMC}$ & 298.944 & -43.648 & 205.2 & 54000 & 59700 & 66000 & 31.00 & 54.47 & 100000 & -6.039 \\
\hline J0449-7031 & LMC & 282.286 & -35.512 & 65.83 & 44000 & 49700 & 56000 & 49.99 & 15.84 & 50463 & -6.184 \\
\hline J0451-67 & LMC & 278.410 & -36.290 & 45 & 44000 & 49700 & 56000 & 45.00 & 0.00 & 4184 & -6.057 \\
\hline J0455-6951 & LMC & 281.290 & -35.187 & 94.89 & 44000 & 49700 & 56000 & 51.13 & 43.76 & 50591 & -6.146 \\
\hline J0456-69 & LMC & 280.457 & -35.334 & 103 & 44000 & 49700 & 56000 & 50.87 & 52.13 & 50514 & -6.113 \\
\hline J0456-7031 & $\mathrm{LMC}$ & 282.049 & -34.966 & 100.3 & 44000 & 49700 & 56000 & 51.53 & 48.77 & 51132 & -6.133 \\
\hline J0457-69 & LMC & 281.143 & -35.113 & 91 & 44000 & 49700 & 56000 & 51.36 & 39.64 & 50356 & -6.138 \\
\hline J0458-67 & LMC & 278.670 & -35.518 & 97 & 44000 & 49700 & 56000 & 50.35 & 46.65 & 50185 & -6.172 \\
\hline J0502-6617 & LMC & 276.869 & -35.504 & 68.9 & 44000 & 49700 & 56000 & 49.98 & 18.92 & 49066 & -6.184 \\
\hline J0519-6932 & LMC & 280.287 & -33.254 & 119.4 & 44000 & 49700 & 56000 & 56.88 & 62.52 & 49741 & -5.803 \\
\hline J0521-68 & LMC & 279.124 & -33.262 & 136 & 44000 & 49700 & 56000 & 56.86 & 79.14 & 49777 & -5.393 \\
\hline J0522-6847 & LMC & 279.348 & -33.168 & 126.45 & 44000 & 49700 & 56000 & 57.17 & 69.28 & 49581 & -5.626 \\
\hline J0529-6652 & LMC & 276.974 & -32.763 & 103.2 & 44000 & 49700 & 56000 & 57.84 & 45.36 & 49581 & -5.937 \\
\hline J0532-6639 & LMC & 276.675 & -32.481 & 69.3 & 44000 & 49700 & 56000 & 58.59 & 10.71 & 47853 & -5.915 \\
\hline J0532-69 & LMC & 280.333 & -32.163 & 124 & 44000 & 49700 & 56000 & 60.40 & 63.60 & 49602 & -5.774 \\
\hline J0534-6703 & LMC & 277.129 & -32.279 & 94.7 & 44000 & 49700 & 56000 & 59.45 & 35.25 & 48820 & -5.890 \\
\hline J0535-66 & LMC & 276.884 & -32.197 & 75 & 44000 & 49700 & 56000 & 59.60 & 15.40 & 48067 & -5.886 \\
\hline J0535-6935 & LMC & 280.076 & -31.936 & 93.7 & 44000 & 49700 & 56000 & 61.18 & 32.52 & 48857 & -5.841 \\
\hline J0537-69 & LMC & 279.768 & -31.730 & 273 & 44000 & 49700 & 56000 & 61.89 & 211.11 & 49901 & -3.633 \\
\hline J0540-6919 & LMC & 279.717 & -31.516 & 146.5 & 44000 & 49700 & 56000 & 62.62 & 83.88 & 48907 & -5.290 \\
\hline J0542-68 & LMC & 278.449 & -31.406 & 114 & 44000 & 49700 & 56000 & 62.85 & 51.15 & 48893 & -5.794 \\
\hline J0543-6851 & LMC & 279.125 & -31.235 & 131 & 44000 & 49700 & 56000 & 63.57 & 67.43 & 48805 & -5.673 \\
\hline J0555-7056 & LMC & 281.460 & -30.119 & 73.4 & 44000 & 49700 & 56000 & 67.41 & 5.99 & 48786 & -5.673 \\
\hline
\end{tabular}


Table A7. Fast Radio Bursts

\begin{tabular}{|c|c|c|c|c|c|c|c|c|c|c|c|c|}
\hline Name & $\begin{array}{c}l \\
\left({ }^{\circ}\right)\end{array}$ & $\begin{array}{c}b \\
\left({ }^{\circ}\right)\end{array}$ & $\begin{array}{c}\mathrm{DM} \\
\left(\mathrm{cm}^{-3} \mathrm{pc}\right)\end{array}$ & $\begin{array}{c}\text { Obs. } \tau_{\mathrm{sc}} \\
(\mathrm{ms})\end{array}$ & Ref. & $\begin{array}{c}\mathrm{DM}_{\mathrm{Gal}} \\
\left(\mathrm{cm}^{-3} \mathrm{pc}\right)\end{array}$ & $\begin{array}{c}\mathrm{DM}_{\mathrm{MC}} \\
\left(\mathrm{cm}^{-3} \mathrm{pc}\right)\end{array}$ & $\begin{array}{c}\mathrm{DM}_{\mathrm{IGM}} \\
\left(\mathrm{cm}^{-3} \mathrm{pc}\right)\end{array}$ & $z$ & $\begin{array}{c}D_{m} \\
(\mathrm{Mpc})\end{array}$ & $\begin{array}{c}\log \tau_{s c} \\
(\mathrm{~s})\end{array}$ & $\begin{array}{c}\tau_{s c} \\
(\mathrm{~ms})\end{array}$ \\
\hline FRB010125 & 356.641 & -20.020 & $790(3)$ & $<1.4$ & bb14 & 75.91 & 0.00 & 614.09 & 0.861 & 2769 & -2.775 & 1.68 \\
\hline FRB010621 & 25.433 & -4.003 & $745(10)$ & $<2.1$ & $\mathrm{kkl}+11$ & 321.56 & 0.00 & 323.44 & 0.453 & 1667 & -2.868 & 1.36 \\
\hline FRB010724 & 300.653 & -41.805 & 375 & 1.2 & $\mathrm{lbm}+07$ & 32.65 & 61.38 & 180.97 & 0.254 & 1008 & -3.465 & 0.34 \\
\hline FRB090625 & 226.443 & -60.030 & $899.55(1)$ & $3.7(7)$ & $\mathrm{cpk}+16$ & 25.48 & 0.00 & 774.07 & 1.085 & 3276 & -2.645 & 2.26 \\
\hline FRB110220 & 50.828 & -54.766 & $944.38(5)$ & $1.9(1)$ & $\mathrm{tsb}+13$ & 24.12 & 0.00 & 820.26 & 1.150 & 3412 & -2.612 & 2.44 \\
\hline FRB110523 & 56.119 & -37.819 & $623.30(6)$ & $4.1(4)$ & $\mathrm{mls}+15$ & 33.00 & 0.00 & 490.30 & 0.687 & 2332 & -2.902 & 1.25 \\
\hline FRB110627 & 355.861 & -41.752 & $723.0(3)$ & $<0.5$ & $\mathrm{tsb}+13$ & 33.57 & 0.00 & 589.43 & 0.826 & 2685 & -2.798 & 1.59 \\
\hline FRB110703 & 80.997 & -59.019 & $1103.6(7)$ & $<1.5$ & $\mathrm{tsb}+13$ & 23.08 & 0.00 & 980.52 & 1.375 & 3855 & -2.511 & 3.08 \\
\hline FRB120127 & 49.287 & -66.203 & $553.3(3)$ & $<0.4$ & $\mathrm{tsb}+13$ & 20.63 & 0.00 & 432.67 & 0.607 & 2114 & -2.973 & 1.06 \\
\hline FRB121002 & 308.219 & -26.264 & $1629.18(2)$ & $6.7(7)$ & $\mathrm{cpk}+16$ & 60.50 & 0.00 & 1468.68 & 2.059 & 4984 & -2.283 & 5.21 \\
\hline FRB121102 & 174.950 & -0.225 & $557(2)$ & $<1.5$ & $\mathrm{sch}+14$ & 287.12 & 0.00 & 169.88 & 0.238 & 952 & -3.074 & 0.84 \\
\hline FRB130626 & 7.450 & +27.420 & $952.4(1)$ & $2.9(7)$ & $\mathrm{cpk}+16$ & 65.09 & 0.00 & 787.31 & 1.104 & 3316 & -2.635 & 2.32 \\
\hline FRB130628 & 225.955 & +30.655 & $469.88(1)$ & $1.24(7)$ & $\mathrm{cpk}+16$ & 46.99 & 0.00 & 322.89 & 0.453 & 1665 & -3.138 & 0.73 \\
\hline FRB130729 & 324.787 & +54.744 & $861(2)$ & $23(2)$ & $\mathrm{cpk}+16$ & 25.42 & 0.00 & 735.58 & 1.031 & 3159 & -2.673 & 2.12 \\
\hline FRB131104 & 260.549 & -21.925 & $779(1)$ & $0.3(1)$ & $\mathrm{rsj} 15$ & 220.20 & 0.00 & 458.80 & 0.643 & 2214 & -2.940 & 1.15 \\
\hline FRB140514 & 50.841 & -54.611 & $562.7(6)$ & $5.4(1)$ & $\mathrm{pbb}+15$ & 24.17 & 0.00 & 438.53 & 0.615 & 2136 & -2.965 & 1.08 \\
\hline FRB150418 & 232.665 & -3.234 & $776.2(5)$ & $<0.25$ & $\mathrm{kjb}+16$ & 325.54 & 0.00 & 350.66 & 0.492 & 1782 & -2.845 & 1.43 \\
\hline
\end{tabular}

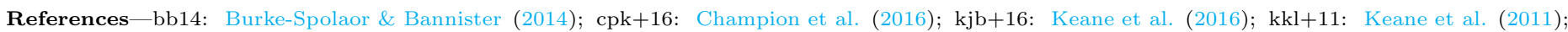


tsb+13: Thornton et al. (2013) 


\section{B. PERPENDICULAR DISTANCES}

In this section we give the equations that are used to compute the perpendicular distances from spiral arms and the Gum Nebula shell. These distances are required to estimate the electron density near these features using the equations given in $\S 3.3$ and $\S 3.5$ respectively. Other features such as the nebula at the Galactic Center (§3.4) and Loop I (§3.7) have circular or spherical symmetry and so the calculations of perpendicular distances are relatively simple as given by the equations in the relevant sub-section of $\S 3$. These equations only approximate the true perpendicular distances from a point $(x, y)$, but their implementations are faster than the more precise (iterative) solutions and are adequate for present purposes.

\section{B.1. Spiral arms}

To compute the $n_{e}$ profile of spiral arms (Equation 11), we require the perpendicular distance $s_{a}$ from the central axis of the spiral arm in the Galactic $(x, y)$ plane. The geometry is illustrated in Figure B.1. The polar coordinates of $(x, y)$ are $(R, \phi)$ where the origin is at the Galactic Center. From Equation 10, $R_{a}$, the radial distance of the point $\left(x_{a}, y_{a}\right)$ on the arm axis at azimuth $\phi$, is given by

$$
R_{a}=R_{a_{i}} \exp \left[\left(\phi-\phi_{a_{i}}\right) \tan \psi_{a}\right]
$$

where $\psi_{a}$ is the pitch angle of the spiral arm and $R_{a_{i}}$ and $\phi_{a_{i}}$ give the start location of the spiral (see Table 1). The approximate perpendicular distance from the arm axis is then given by:

$$
s_{a}=\left(R-R_{a}\right) \cos \psi_{a}
$$

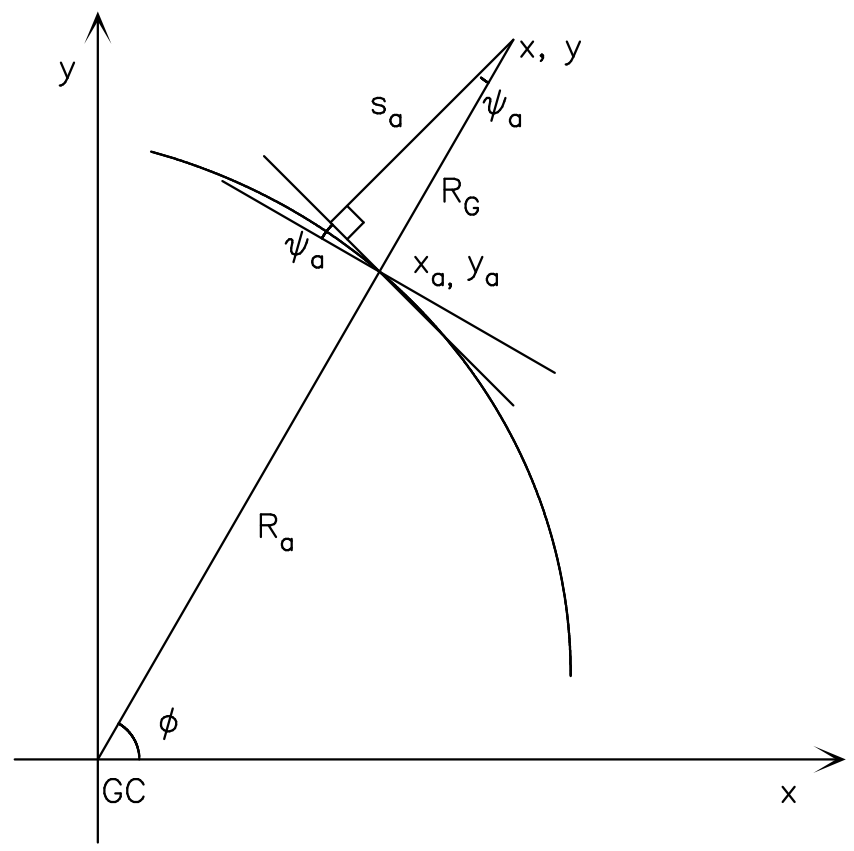

Figure B.1. Geometry for estimating the perpendicular distance from a point $(x, y)$ in the plane of the Galaxy to the central axis of a spiral arm. 


\section{B.2. Gum Nebula}

We model the Gum Nebula as an ellipsoidal shell with major axis in the $z$ direction where the local $(x, y, z)$ system is centered on the Gum Nebula and these axes are parallel to the corresponding Galactic axes. We require the perpendicular distance to the shell mid-line from either inside or outside the shell to compute the local electron density (Equation 15). The shell is circular in the $x-y$ plane and hence, for the purposes of this calculation, we simplify the problem to two dimensions, with the $x-z$ plane passing through the point of interest. Figure B.2 shows the geometry in the $x-z$ plane. For compactness we use $a$ and $b$ for the semi-minor axis and semi-major axis, respectively.

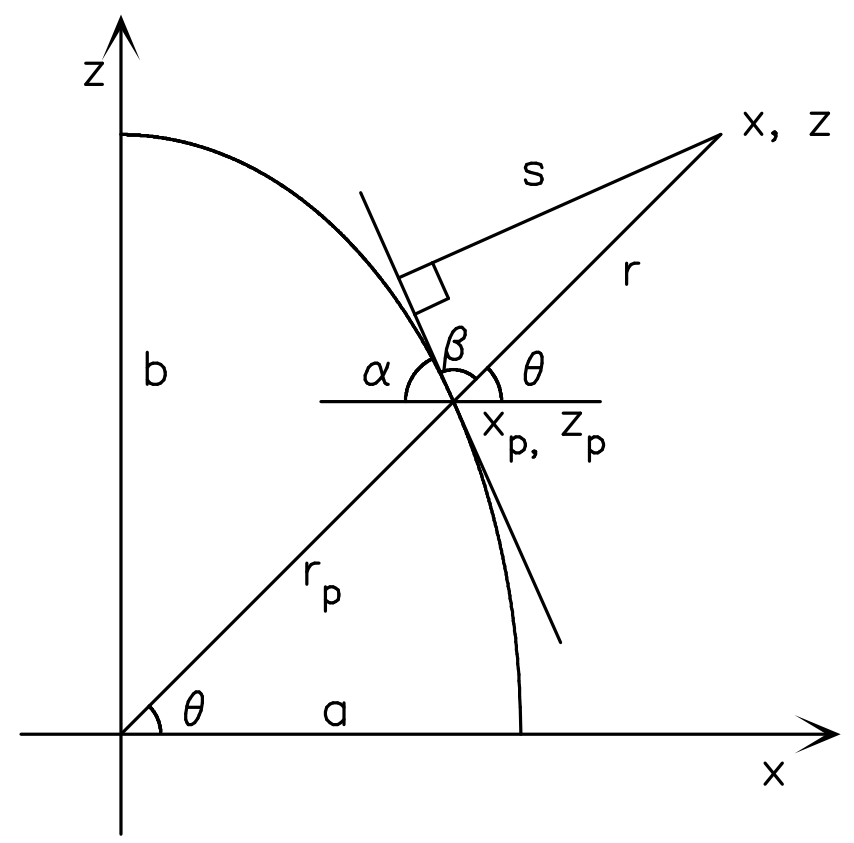

Figure B.2. Geometry of the Gum Nebula model. The origin of this local coordinate system is the center of the Nebula with the $z$ axis is parallel to the Galactic $z$ axis.

A point $\left(x_{p}, z_{p}\right)$ on the shell is then given by:

$$
\frac{x_{p}^{2}}{a^{2}}+\frac{z_{p}^{2}}{b^{2}}=1
$$

and we require the perpendicular distance $s$ from a point $(x, z)$ to the shell mid-line. The polar angle $\theta$ is given by:

$$
\tan \theta=\frac{z}{x}=\frac{z_{p}}{x_{p}}
$$

Given $(x, z)$, we can then obtain $x_{p}$ and $z_{p}$ as follows:

$$
\begin{aligned}
& x_{p}=\frac{a b}{\left(b^{2}+a^{2} \tan ^{2} \theta\right)^{1 / 2}} \\
& z_{p}=\frac{a b \tan \theta}{\left(b^{2}+a^{2} \tan ^{2} \theta\right)^{1 / 2}}
\end{aligned}
$$

From Figure B.2, $\tan (-\alpha)$ is the gradient of the tangent at $\left(x_{p}, z_{p}\right)$ :

$$
\tan (-\alpha)=\frac{-b x_{p}}{a\left(a^{2}-x_{p}^{2}\right)^{1 / 2}}
$$

the angle $\beta$ is:

$$
\beta=180^{\circ}-\alpha-\theta
$$

and

$$
r-r_{p}=\left[\left(x-x_{p}\right)^{2}+\left(z-z_{p}\right)^{2}\right]^{1 / 2} .
$$


We can then approximate the perpendicular distance from $(x, z)$ to the shell mid-line by:

$$
s=\left(r-r_{p}\right) \sin \beta \text {. }
$$




\section{COORDINATE SYSTEMS FOR THE LARGE MAGELLANIC CLOUD}

To compute electron densities in the Large Magellanic Cloud we need to transform from the Galactic $(l, b, D)$ system to the $\left(x^{\prime}, y^{\prime}, z^{\prime}\right)$ system centered on the LMC with the $x^{\prime}$ axis along the line of nodes as described in $\S 3.9$. Figure C.1 illustrates the relevant axes and angles.

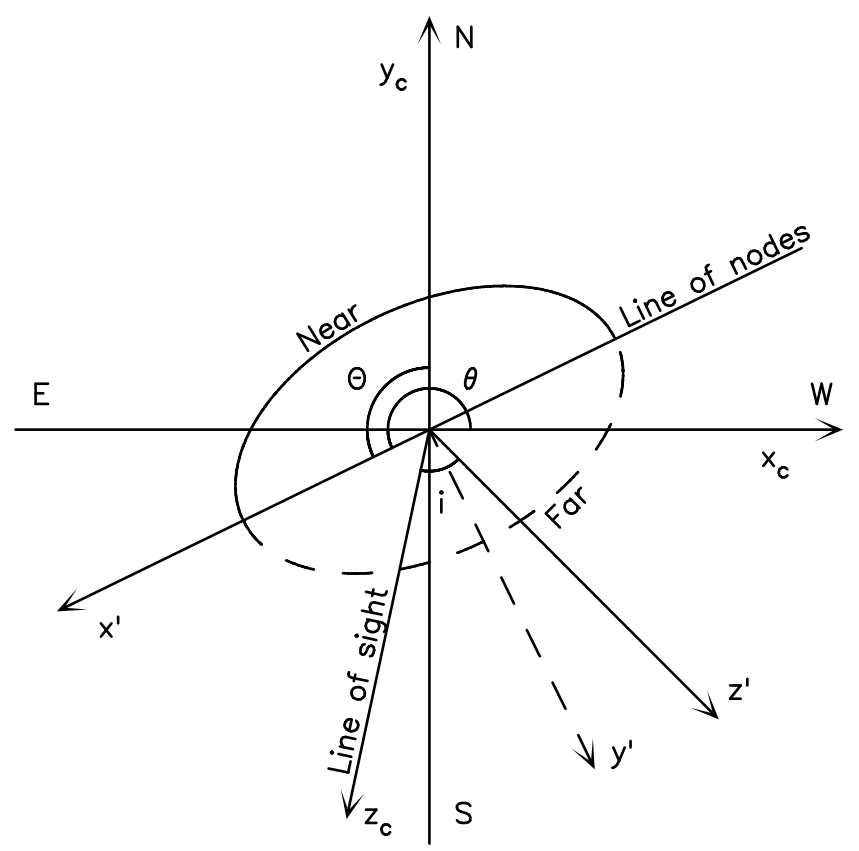

Figure C.1. Coordinate systems for the Large Magellanic Cloud. The $\left(x_{c}, y_{c}, z_{c}\right)$ system is centered on the LMC with the $+x_{c}$ axis in the direction of decreasing right ascension (W), the $+y_{c}$ axis toward north and the $+z_{c}$ axis toward the observer. The $\left(x^{\prime}, y^{\prime}, z^{\prime}\right)$ system is also centered on the LMC with the galaxy disk in the $\left(x^{\prime}, y^{\prime}\right)$ plane and $+x^{\prime}$ along the line of nodes at position angle $\theta$ measured counter-clockwise from the $x_{c}$ axis. The $+y^{\prime}$ axis lies behind the plane of the sky and the disk normal $+z^{\prime}$ is oriented at inclination angle $i$ to the $z_{c}$ axis in front of the plane of the sky.

Since the parameters of the LMC are described in celestial (J2000) coordinates, we first convert to the $(\alpha, \delta, D)$ system using: $\mathrm{LMC}\left(\alpha_{\mathrm{LMC}}, \delta_{\mathrm{LMC}}\right)$ and $\phi$ is a position angle measured counter-clockwise from the direction of decreasing right ascension:

$$
\begin{aligned}
\cos \rho & =\cos \delta \cos \delta_{\mathrm{LMC}} \cos \left(\alpha-\alpha_{\mathrm{LMC}}\right)+\sin \delta \sin \delta_{\mathrm{LMC}} \\
\sin \rho \cos \phi & =-\cos \delta \sin \left(\alpha-\alpha_{\mathrm{LMC}}\right) \\
\sin \rho \sin \phi & =\sin \delta \cos \delta_{\mathrm{LMC}}-\cos \delta \sin \delta_{\mathrm{LMC}} \cos \left(\alpha-\alpha_{\mathrm{LMC}}\right)
\end{aligned}
$$

The $\left(x_{c}, y_{c}, z_{c}\right)$ system is then defined by:

$$
\begin{aligned}
& x_{c}=D \sin \rho \cos \phi \\
& y_{c}=D \sin \rho \sin \phi \\
& z_{c}=D \mathrm{LMC}-D \cos \rho
\end{aligned}
$$

and the $\left(x^{\prime}, y^{\prime}, z^{\prime}\right)$ system by:

$$
\begin{aligned}
& x^{\prime}=x_{c} \cos \theta+y_{c} \sin \theta \\
& y^{\prime}=-x_{c} \sin \theta \cos i+y_{c} \cos \theta \cos i-z_{c} \sin i \\
& z^{\prime}=-x_{c} \sin \theta \sin i+y_{c} \cos \theta \sin i+z_{c} \cos i .
\end{aligned}
$$

where $i$ is the LMC plane inclination angle, $\theta=\Psi+90^{\circ}$ and $\Psi$ is the astronomical position angle of the line of nodes. Using Equations C12 and C13 we obtain:

$$
\begin{aligned}
& x^{\prime}=D \sin \rho \cos (\phi-\theta) \\
& y^{\prime}=D[\sin \rho \cos i \sin (\phi-\theta)+\cos \rho \sin i]-D_{\mathrm{LMC}} \sin i \\
& z^{\prime}=D[\sin \rho \sin i \sin (\phi-\theta)-\cos \rho \cos i]+D_{\mathrm{LMC}} \cos i
\end{aligned}
$$




\section{THE YMW16 DISTANCE - DM PROGRAM}

The program YMW16 computes distances for Galactic pulsars, Magellanic Cloud pulsars and Fast Radio Bursts (FRBs) from their Galactic coordinates and DMs using the YMW16 model parameters. It also does the reverse calculation, computing DMs that correspond to given Galactic coordinates and distances. An estimate of the scattering timescale $\tau_{\mathrm{sc}}$ is output for Galactic and Magellanic Cloud pulsars and FRBs.

The program is written in $\mathrm{C}$ and is publically available at the following websites:

- http://www.xao.ac.cn/ymw16/

- http://www.atnf.csiro.au/research/pulsar/ymw16/

- https://bitbucket.org/psrsoft/ymw16/

The first two websites also have an interactive facility enabling on-line execution of the YMw16 program and provide for download of the latest version of the program. The third website includes the full development history of the program, a download facility and an "issues" reporting system.

Following the definitions in section 3, YMw16 includes eight functions for Galactic components, three for Magellanic Cloud components and one for FRBs. The corresponding $\mathrm{C}$ files are thick.c, thin.c, spiral.c, galcen.c, gum.c, localbubble.c, nps.c, fermibubble.c, lmc.c, dora.c, smc.c and frb_d.c. The two remaining $\mathrm{C}$ files are the main program ywm16.c and ymw16par.c which reads in the model parameters (Table 2) from ymw16par.txt. The spiral parameters (Table 1) are contained in spiral.txt which is read in by spiral.c. The Sun is located at $z_{\odot}=+6.0 \mathrm{pc}$ above the Galactic plane and a warp in the outer Galactic disk is included in the model.

To compute the distance $D_{m}$ corresponding to a given DM, the local $n_{e}$ is evaluated at steps of 5 pc along the path, or steps of $D_{t} / 200$ if the nominal number of steps along the path is less than 200 , where $D_{t}=\mathrm{DM} / 0.013 \mathrm{pc}$ is a nominal distance. The model DM is accumulated at each step until the input DM is reached (or the distance limit is reached). For the inverse process, the DM is accumulated out to the input distance. In order to improve the computational efficiency of the program, we cease calculation of all model components when the distance from the central point or axis of a given component is greater than six times the scale length of that component. For a sech ${ }^{2}$ dependence, the value of $n_{e}$ at this point is less than $10^{-5}$ times the central value and hence negligible.

To compile and execute the YMW16 program:

1. Download the source code from one of the above websites and unpack.

2. Run make_ymw16 to compile the code and create the executable Ymw16.

3. To run YMw16: ymw16 [-h] [-t $\langle$ text $\rangle][-\mathrm{d}\langle$ dirname $\rangle][-\mathrm{v}][-\mathrm{V}]\langle$ mode $\rangle\langle\mathrm{gl}\rangle\langle\mathrm{gb}\rangle\langle$ DM/Dist $\rangle$ [ DM_Host $\rangle]$ $\langle$ ndir $\rangle$, where optional inputs are enclosed in square brackets and

-h: prints the help page

-t $\langle$ text $\rangle$ : where $\langle$ text $\rangle$ (no space, maximum 64 characters) is appended to the output line

-d $\langle$ dirname $\rangle$ : where $\langle$ dirname $\rangle$ is a directory containing YMW16 data files

-v: prints diagnostics

-V: prints more diagnostics

mode is one of: Gal, MC, or IGM

gl: Galactic longitude (deg.)

gb: Galactic latitude (deg.)

DM/Dist: One of DM $\left(\mathrm{cm}^{-3} \mathrm{pc}\right)$ or distance, depending on ndir. Distance has units of pc for modes Gal and MC and Mpc for mode IGM

DM_Host: Dispersion measure of the FRB host galaxy in the observer frame (default $100 \mathrm{~cm}^{-3} \mathrm{pc}$ ). (Note: if present, DM_Host is ignored for Gal and MC modes.)

ndir: ndir $=1$ converts from DM to distance and ndir $=2$ converts from distance to DM. The output $\tau_{\text {sc }}$ has units of seconds.

Output formats are as follows.

For ndir $=1$ :

Gal: $\mathrm{gl}=\langle\mathrm{val}\rangle \mathrm{gb}=\langle\mathrm{val}\rangle \mathrm{DM}=\langle\mathrm{val}\rangle \mathrm{DM}_{-} \mathrm{Gal}:\langle\mathrm{val}\rangle$ Dist: $\langle\mathrm{val}\rangle \log \left(\mathrm{tau}_{\mathrm{sc}}\right):\langle\mathrm{val}\rangle\langle\mathrm{text}\rangle$

MC: $\mathrm{gl}=\langle\mathrm{val}\rangle \mathrm{gb}=\langle\mathrm{val}\rangle \mathrm{DM}=\langle\mathrm{val}\rangle \mathrm{DM} \_G a l:\langle\mathrm{val}\rangle \mathrm{DM} \_\mathrm{MC}:\langle\mathrm{val}\rangle$ Dist: $\langle\mathrm{val}\rangle \log ($ tau_sc $):\langle\mathrm{val}\rangle\langle$ text $\rangle$

IGM: $\mathrm{gl}=\langle\mathrm{val}\rangle \mathrm{gb}=\langle\mathrm{val}\rangle \mathrm{DM}=\langle\mathrm{val}\rangle$ DM_Gal : $\langle\mathrm{val}\rangle$ DM_MC: $\langle\mathrm{val}\rangle$ DM_IGM: $\langle$ val $\rangle$ DM_Host: $\langle$ val $\rangle \mathrm{z}:\langle$ val $\rangle$ 
Dist: $\langle$ val $\rangle \log ($ tau_sc $):\langle$ val $\rangle\langle$ text $\rangle$.

For ndir $=2$ :

Gal: $g l=\langle$ val $\rangle \mathrm{gb}=\langle\mathrm{val}\rangle \mathrm{D}=\langle\mathrm{val}\rangle \mathrm{DM}:\langle\mathrm{val}\rangle \log ($ tau_sc $):\langle\mathrm{val}\rangle\langle$ text $\rangle$

MC: $g l=\langle v a l\rangle g b=\langle v a l\rangle D=\langle$ val $\rangle$ DM_Gal: $\langle$ val $\rangle$ DM_MC: $\langle$ val $\rangle \mathrm{DM}:\langle$ val $\rangle \log ($ tau_sc $):\langle$ val $\rangle\langle$ text $\rangle$

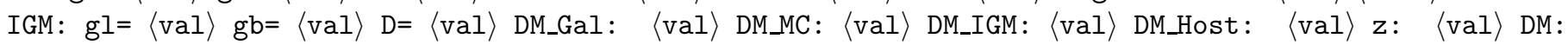

$\langle$ val $\rangle \log ($ tau_sc $):\langle$ val $\rangle\langle$ text $\rangle$.

For ndir $=1$ and mode Gal, if the input DM exceeds the range of the Galactic model, the distance is set set to $25000 \mathrm{pc}$. For ndir $=1$ and mode MC, the upper limit is $100000 \mathrm{pc}$. There is no upper limit for IGM mode. 